December 15, 1994

LBL-36537

UCB-PTH-94/35

\title{
Differential Geometry on Hopf Algebras and Quantum Groups
}

(Ph.D. Thesis $)^{1}$

\author{
Paul Watts ${ }^{2}$ \\ Department of Physics \\ University of California \\ and \\ Theoretical Physics Group \\ Lawrence Berkeley Laboratory \\ University of California \\ Berkeley, CA 94720
}

\begin{abstract}
The differential geometry on a Hopf algebra is constructed, by using the basic axioms of Hopf algebras and noncommutative differential geometry. The space of generalized derivations on a Hopf algebra of functions is presented via the smash product, and used to define and discuss quantum Lie algebras and their properties. The Cartan calculus of the exterior derivative, Lie derivative, and inner derivation is found for both the universal and general differential calculi of an arbitrary Hopf algebra, and, by restricting to the quasitriangular case and using the numerical $R$-matrix formalism, the aforementioned structures for quantum groups are determined.
\end{abstract}

\footnotetext{
${ }^{1}$ This work was supported in part by the Director, Office of Energy Research, Office of High Energy and Nuclear Physics, Division of High Energy Physics of the U.S. Department of Energy under Contract DE-AC03-76SF00098 and in part by the National Science Foundation under grant PHY-90-21139.

${ }^{2}$ e-mail: watts@cpt.univ-mrs.fr 


\section{DISCLAIMER}

This report was prepared as an account of work sponsored by an agency of the United States Government. Neither the United States Government nor any agency thereof, nor any of their employees, make any warranty, express or implied, or assumes any legal liability or responsibility for the accuracy, completeness, or usefulness of any information, apparatus, product, or process disclosed, or represents that its use would not infringe privately owned rights. Reference herein to any specific commercial product, process, or service by trade name, trademark, manufacturer, or otherwise does not necessarily constitute or imply its endorsement, recommendation, or favoring by the United States Government or any agency thereof. The views and opinions of authors expressed herein do not necessarily state or reflect those of the United States Government or any agency thereof. 


\section{DISCLAIMER}

Portions of this document may be illegible in electronic image products. Images are produced from the best available original document. 


\section{Contents}

1 Introduction 1

1.1 A Brief Outline . . . . . . . . . . . . . . 1

1.2 Why Quantum Groups? . . . . . . . . . . . . . . 2

1.2.1 Mathematical and Physical Motivations ......... 2

1.2.2 Quantum Group Symmetry in Field Theory . . . . . . . 2

1.2.3 Lattices and Regularization . . . . . . . . . . . . 3

1.2 .4 Quantum Gravity . . . . . . . . . . . . . . . 4

1.3 Our Approach . . . . . . . . . . . 5

2 Hopf Algebras

2.1 Basic Definitions . . . . . . . . . . . . 7

2.2 Dually Paired Hopf Algebras . . . . . . . . . . . . . . 9

2.3 Representations of Hopf Algebras and Quantum Groups ..... . 9

2.4 Examples . . . . . . . . . . . . . . . . . 12

2.4.1 Classical Lie Algebras . . . . . . . . . . . . . 12

2.4 .2 The Hopf Algebra $U_{q}(s u(2)) \ldots \ldots \ldots \ldots \ldots . \ldots . \ldots 12$

2.4.3 The Quantum Group $G L_{q}(2) \ldots \ldots \ldots \ldots \ldots$

2.5 Sweedler's Notation . . . . . . . . . . . . . . . . 14

3 Actions, the Smash Product, and Coactions 16

3.1 Actions and Generalized Derivations $\ldots \ldots \ldots \ldots \ldots$

3.2 The Smash Product . . . . . . . . . . . . . . . . . 17

3.2.1 Example: The Haar Measure and the Smash Product . . . . 19

3.3 Coactions ...................... 21

3.4 Actions and Coactions on the Smash Product . . . . . . . 22

3.4.1 Bicovariance of the Smash Product . . . . . . . . . 23

3.4.2 The Canonical Element of $\mathcal{A} \rtimes \mathcal{U} \ldots \ldots \ldots$ 
3.5 Example: The 2-Dimensional Quantum Euclidean Group . . . . . 27

4 Quantum Lie Algebras 31

4.1 Basics of Quantum Lie Algebras . . . . . . . . . . . . . . 31

4.2 The Adjoint Representation . . . . . . . . . . . . . . 33

4.3 Quasitriangular Quantum Lie Algebras . . . . . . . . . . . . . 34

4.3.1 Example: $U_{q}(s l(2)) \ldots \ldots \ldots \ldots \ldots \ldots \ldots$

4.4 The Killing Metric . . . . . . . . . . . . . . . . . 39

4.4.1 The Killing Form for a Quasitriangular Hopf Algebra . . . . 39

4.4.2 The Killing Metric for a Quantum Lie Algebra . . . . . . . 40

4.4 .3 Examples . . . . . . . . . . . . . . . 42

4.5 Some Comments on the Adjoint Representation . . . . . . . 45

5 Cartan Calculus on Hopf Algebras and Quantum Lie Algebras 47

5.1 Universal Cartan Calculus . . . . . . . . . . . . . . 47

5.1 .1 Cartan-Maurer Forms . . . . . . . . . . . 51

5.1 .2 General Cartan Calculus . . . . . . . . . . . . 52

5.2 Cartan Calculus for Quantum Lie Algebras . . . . . . . . . . 53

5.2 .1 The Quasitriangular Case ............. 54

6 The Linear Quantum Groups $G L_{q}(N)$ and $S L_{q}(N) \quad 56$

6.1 The Quantum Plane and the Quantum Determinant . . . . . . . 56

6.1.1 The Cartan Calculus for $G L_{q}(N) \ldots \ldots \ldots \ldots$

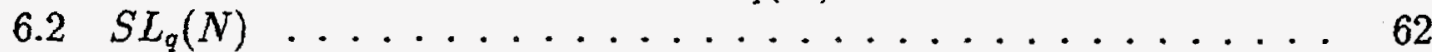

6.2 .1 The Quantum Group $S L_{q}(N) \ldots \ldots \ldots \ldots \ldots \ldots$

6.2 .2 The Calculus for $S L_{q}(N) \ldots \ldots \ldots \ldots \ldots \ldots$

7 Conclusions $\quad 65$

7.1 The Killing Metric . . . . . . . . . . . . . . . . . 65

7.2 Inner Derivations for a General Cartan Calculus . . . . . . . . 66

$7.3 S O_{q}(N)$ and $S P_{q}\left(\frac{1}{2} N\right) \ldots \ldots \ldots \ldots \ldots \ldots \ldots \ldots$

7.4 Fiber Bundles and Deformed Gauge Theories . . . . . . . . 68

$\begin{array}{ll}\text { Bibliography } & 69\end{array}$

A Numerical R-Matrix Relations $\quad \mathbf{7 2}$

A.1 The Element $u$. . . . . . . . . . . . . . . . 72

A.2 R-Matrices for the Simple Lie Algebras . . . . . . . . . 74 
A.2.1 R- and D-Matrices . . . . . . . . . . . 74

A.2.2 Characteristic Equations and Trace Relations . . . . . 75

A.2.3 Projectors .................. 76

B Classical Differential Geometry $\quad 78$

B.1 The Tangent Space . . . . . . . . . . . . . 78

B.2 The Exterior Derivative $\ldots \ldots \ldots \ldots \ldots$

B.3 The Inner Derivation . . . . . . . . . . . . . 80

B.4 The Lie Derivative and the Graded Derivation Algebra . . . . . . 81

C Differential Calculus on Hopf Algebras $\quad 82$

C.1 The Universal Differential Calculus . . . . . . . . . . . . 82

C.1.1 The Universal Differential Calculus of a Hopf Algebra . . . . 83

C.2 General Differential Calculus . . . . . . . . . . . . . 84 


\section{Acknowledgements}

First and foremost, I wish to thank my research advisor Bruno Zumino, whose guidance and encouragement has been invaluable to me; it is literally impossible to thank him enough for all his help, and this work could not exist without his many contributions. I must also recognize my fellow partners-in-crime Peter Schupp and Chryss Chryssomalakos, without whom I would be considerably poorer in both friendship and knowledge.

There are naturally many others who I must thank; among my fellow physics grad students here at Berkeley, I have benefitted immeasurably from conversations with Wati Taylor and Scott Hotes. Rich Lebed certainly deserves special mention for being able to share an office with my whining and pedantry for several years, and Steve Johnson and Peter Grudberg should get medals for being able to live with me for what to them probably seems like an eternity. Among the postdocs, Markus Luty, Michael Schlieker and Joanne Cohn, as well as Markus Pflaum in the Math Department, all deserve my gratitude for their friendship and encouragement.

I have gained quite a lot from my interactions with many of the faculty here at Cal, but I would particularly like to thank Orlando Alvarez for numerous fascinating discussions, and to Korkut Bardakçi and Nicolai Reshetikhin for agreeing to read and approve this work.

There is no doubt that I have survived grad school largely due to the diligence and assistance of the LBL Theory Group staff, Betty Moura and Luanne Neumann, as well as their counterparts on campus, Anne Takizawa and Donna Sakima. They have been infinitely helpful in their efforts to make the LBL and UCB bureaucracies (almost) tolerable.

Last, but by no stretch of the imagination least, I must thank my parents Jean and Bob Watts, for being very atypical military parents; their willingness to let me follow my own muse and their support of the path I have chosen has given me the utmost respect for them. They did a damn fine job with me and my siblings. 
This work was supported in part by the Director, Office of Energy Research, Office of High Energy and Nuclear Physics, Division of High Energy Physics of the U.S. Department of Energy under Contract DE-AC03-76SF00098 and in part by the National Science Foundation under grant PHY-90-21139. 


\section{Chapter 1}

\section{Introduction}

\subsection{A Brief Outline}

In the present chapter; we hope to present to the reader the motivation behind this work, and what we hope to accomplish herein. The next two chapters, "Hopf Algebras" and "Actions, the Smash Product, and Coactions", are largely introductory, and serve to establish the language, notations, and methods which we use throughout; once we have these foundations, we are able to build upon them in the subsequent chapters to obtain the more advanced and original results which form the core of this work. Chapter 4, "Quantum Lie Algebras", deals with a particular class of Hopf algebras and examines their structures, with an emphasis finding the deformed analogues of classical concepts (e.g. the Killing metric). The following chapter, "Cartan Calculus on Hopf Algebras and Quantum Lie Algebras" examines in depth the differential geometry of the titular objects, by introducing an algebra of generalized derivations, and using many of the concepts of noncommutative geometry to examine the structure of this algebra. In Chapter 6, "The Linear Lie Groups $G L_{q}(N)$ and $S L_{q}(N)$ ", we apply the results of the preceding chapters to these two specific cases. Finally, in "Conclusions", we mention what other problems might be constructively pursued using the ideas here presented, and what difficulties might arise in so doing.

There are also three appendices, "Numerical R-Matrix Relations", "Classical Differential Geometry", and "Differential Calculus on Hopf Algebras"; these cover topics which the reader may already be familiar with, but which serve as introductions to the relevant material if he/she is not. 


\subsection{Why Quantum Groups?}

The reader may wonder why any of the material contained herein would be of any interest or use to a physicist. In the following subsections, we hope to give some possible instances where the results of this work might prove to be useful.

\subsubsection{Mathematical and Physical Motivations}

Perhaps most importantly, Hopf algebras (and quantum groups, which are specific types of Hopf algebras) provide us with a generalization of many of the algebras which are common in physics. (For instance, as we describe in one of the examples in Chapter 2, any Lie algebra is actually a Hopf algebra, albeit with a somewhat trivial structure in many ways.) Furthermore, they also allow us to generalize many "classical" physical ideas to "deformed" versions in a completely self-consistent manner. The new versions are most often specified by one or more parameters, and the classical case is recovered by setting these parameters to some fixed values. In this sense, the situation is much like quantum mechanics as a "deformed" version of classical mechanics described by Planck's constant $h$, with the latter being recovered in the $h \rightarrow 0$ limit (in fact, this was the motivation for the term "quantum group"). The reader will encounter many of these generalizations throughout this work, such as the quantum group $S L_{q}(N)$, which is a "deformed" version of the "classical" $S L(N)$, which is recovered in the limit $q \rightarrow 1$.

The language of Hopf algebras also gives us a way of "rephrasing" many of the ideas and concepts which are used extensively in physics in more mathematical terms. In many cases, we feel that this not only eases computations, but also provides some insight into a more general structure of the problem in question. For instance, in Chapter 3, we describe how the common physical concepts of finite and infinitesimal transformations may be recast in terms of "coactions" and "actions" of a Hopf algebra on a vector space. We also show how the commutation relations between differential operators and functions, i.e. how the former "act on" and "move through" the latter, also have a straightforward mathematical interpretation in terms of the so-called "smash product".

\subsubsection{Quantum Group Symmetry in Field Theory}

In physics, we often encounter systems which have certain global symmetries, and when we formulate a way of describing these systems, our formulation must respect 
these symmetries. However, we might consider a theory in which one of the symmetry groups is not a classical group, but rather a quantum group. Such a theory will contain the classical theory as a specific case, of course, but will have more degrees of freedom to play with, namely the parameter(s) of deformation which characterize the quantum group.

The first thing we could do with a theory like this is to try to measure these extra parameters experimentally. Suppose we have a lagrangian which is a scalar under transformations in some quantum group, e.g. $S U_{q}(2)$. We could use standard techniques to calculate various physical processes, such as scattering amplitudes or decay rates; these would then have some dependence on the parameter $q$, and by actually running experiments which would test the predictions of this theory, its value could be determined. In this sense, it would be just another constant in the theory.

However, consider the case where we believe the lagrangean to be invariant under the action of the classical group being considered (e.g. $S U(2)$ ). Then the interpretation of the quantum symmetry would be as a method of explicitly breaking the symmetry of the lagrangean, with the degree of breaking parametrized by $q-1$. This quantity would then serve as a measure of how much the actual symmetry deviates from the expected symmetry (in this sense, it is much like the parameter $\epsilon$ which characterizes CP-violation).

\subsubsection{Lattices and Regularization}

Consider a space whose points are described by some set of coordinates, and a set of coordinate transformations described by a group of matrices; we normally assume that these coordinates commute with each other, and therefore, if the transformations are to respect this commutativity, the entries of the transformation matrices will commute as well. However, if we now want to generalize to the case that the matrices may be representations of a quantum group, their entries will in general no longer be commutative, and therefore the coordinates on the space will not remain so either. It is no surprise that the derivatives with respect to these coordinates also no longer commute.

However, what may be surprising is that when one of these differential operators acts on a function (i.e. a sum of ordered monomials), the result is not a normal derivative, but rather a finite difference between the function evaluated at two discrete points. (See Chapter 3.5, as well as [1], for an explicit example of this.) Therefore, requiring that the transformations on the coordinates be given by a 
quantum group rather than a classical one also implies that the space itself can be thought of as a lattice of discrete points, with the spacing depending on the deformation parameter(s) characterizing the quantum group.

One of the uses of putting any field theory on a lattice is that the lattice spacing $a$ becomes a parameter which incorporates the small-scale behavior of the theory. Since this behavior is often responsible for divergences which arise, $a$ is often used as a regularization parameter: explicit $a$-dependent counterterms are put into the theory so as to cancel any infinities in the $a \rightarrow 0$ limit. We see that we have an exact analogue of this; a lattice which in a certain limit ( $a \rightarrow 0$ versus quantum group $\rightarrow$ classical group) becomes "real space" (i.e. a continuous space for lattices, commuting coordinates for quantum groups). Therefore, deforming a classical coordinate transformation group provides a natural lattice with which to work, and therefore perhaps a way of handling small-scale behavior of a theory.

\subsubsection{Quantum Gravity}

An intriguing possibility is that noncommutive algebras like the ones we consider in this work may hold the key to dealing with the age-old (well, decades-old) problem of how to incorporate gravity into a quantum field theory. There are two main reasons for thinking this to be the case:

First, an obstacle in quantizing gravity has always been that all our previous field theories place space-time on a special footing. Generally, when we consider a field theory, we introduce an algebra of objects with given commutation relations, and a Hilbert space of states on which they have an action. The operators themselves are taken to live in various representation of some particluar set of symmetry groups, one of which is the group of diffeomorphisms on the underlying manifold which describes the space-time. Therefore, we talk about scalar particles, vector particles, etc., depending on how they transform under a given coordinate transformation. However, this formulation gives the space-time of the theory the mere status of an index space labelling the operators.

This is a big problem if we now want to consider the geometry of space-time as itself described by an operator in the algebra, because we can no longer use the points of the manifold to label the fields. Therefore, it would be nice to eliminate any reference to the underlying space-time, and describe all fields as algebraic objects. There does in fact seem to be a way to do this; a theorem of Gel'fand states (roughly) that an associative algebra with unit is isomorphic to the algebra of functions over some topological space. This is precisely what we want, since it allows us to switch 
topological considerations like general coordinate invariance into purely algebraic language; we merely specify all the commutation relations of our field operators and how they act on the Hilbert space, and determine what space-times have this as a function algebra. Since the operators will in general not commute, this obviously allows us to use the ideas and techniques of noncommutative geometry.

The second reason that the noncommutative structures we consider may be relevant to quantizing gravity stems from the discussion on lattices in the previous subsection, namely, allowing for noncommuting coordinates on the space in question in effect discretizes it. There have always been problems with the nonrenormalizability of gravity due to Planck-scale effects; as soon as one starts to consider distances less than this, all hell breaks loose, and the theory becomes divergent. One solution to this would be to propose that space-time itself is discretized, with the distance between points being around the Planck length. We would want some sort of mechanism which would explain such a structure rather than taking it as an ad hoc assumption, and the noncommutativity of coordinates does this very naturally.

\subsection{Our Approach}

The main emphasis of this work will be on developing a constructive method for introducing differential geometric structures on Hopf algebras using, at first, only the basic axioms of Hopf algebras. This implies that we do not $\grave{a}$ priori assume any particular multiplicative structure on the Hopf algebras in question, and therefore use the techniques developed by Connes [2] for constructing the universal differential calculus of a unital associative algebra. However, since we consider Hopf algebras, we have additional structure, and may use the results of Woronowicz [3] in dealing with the differential geometry. However, when we consider the physically interesting case where the Hopf algebra is actually a quantum group with a given numerical Rmatrix, there are, in fact, given commutation relations, and the work of Reshetikhin, Takhtadzhyan, and Faddeev [4] becomes extremely useful.

The reason for taking this approach lies in the eventual goal, namely, the formulation of a quantum field theory with a quantum group as a gauge symmetry. Since gauge theories are equivalent to looking at a fiber bundle whose connection is the gauge field and where matter fields are merely sections, finding a way of deforming said bundles seems like the most promising way to specify a deformed gauge theory. Since a connection is a 1-form over a bundle, and actions are by definition intergals of forms over the base space of the bundle, it becomes paramount to analyze 
the differential geometry before proceeding. In particular, by using sheaf theoretic techniques in which the topological properties of the manifolds involved become algebraic properties of their function spaces ( $c f$. Appendix B and references therein), we find that the structure group is described by a quantum group, so determining the corresponding Lie algebra and Cartan calculus is necessary for any discussion involving connections. We hope that the results we obtain will further us toward this goal. 


\section{Chapter 2}

\section{Hopf Algebras}

In this chapter, we look at many of the basic properties of Hopf algebras and quantum groups. This is not only to introduce the concepts needed in the manipulations of these mathematical objects, but to also establish much of the notation which will appear throughout this work. (For the interested reader, much more information about Hopf algebras and their properties is readily available in $[5,6,7]$.)

\subsection{Basic Definitions}

An algebra is a vector space $\mathcal{A}$ over a field $k$ such that the algebra multiplication $m: \mathcal{A} \otimes \mathcal{A} \rightarrow \mathcal{A}$ is a bilinear map satisfying

$$
\begin{aligned}
& m(a \otimes(b+c))=m(a \otimes b)+m(a \otimes c), \\
& m((a+b) \otimes c)=m(a \otimes c)+m(b \otimes c),
\end{aligned}
$$

for all $a, b, c \in \mathcal{A}$. (In general, we will suppress $m$ for purposes of brevity, writing $a b$ instead of $m(a \otimes b)$.)

A unital algebra is an algebra which contains an element $1_{\mathcal{A}}$ having the properties

$$
1_{\mathcal{A}} a=a 1_{\mathcal{A}}=a .
$$

An associative algebra is an algebra in which $m$ satisfies the further condition

$$
(a b) c=a(b c) .
$$


A coalgebra is a vector space $\mathcal{A}$ over a field $k$, together with linear maps $\Delta$ : $\mathcal{A} \rightarrow \mathcal{A} \otimes \mathcal{A}$ and $\epsilon: \mathcal{A} \rightarrow k$ (the coproduct and counit, respectively) which satisfy

$$
\begin{aligned}
(\Delta \otimes \mathrm{id}) \Delta(a) & =(\mathrm{id} \otimes \Delta) \Delta(a), \\
(\epsilon \otimes \mathrm{id}) \Delta(a) & =(\mathrm{id} \otimes \epsilon) \Delta(a)=a .
\end{aligned}
$$

In analogy with the associative algebra case, the first of these is often referred to as coassociativity.

A bialgebra is both a unital associative algebra and a coalgebra, with the compatibility conditions that $\Delta$ and $\epsilon$ are both algebra maps with $\Delta\left(1_{\mathcal{A}}\right)=1_{\mathcal{A}} \otimes 1_{\mathcal{A}}$ and $\epsilon\left(1_{\mathcal{A}}\right)=1_{k}$.

A Hopf algebra is a bialgebra together with a linear map $S: \mathcal{A} \rightarrow \mathcal{A}$, the antipode, which satisfies

$$
m((S \otimes \mathrm{id}) \Delta(a))=m((\mathrm{id} \otimes S) \Delta(a))=\epsilon(a) 1_{\mathcal{A}} .
$$

It follows that the antipode is an antialgebra map, i.e. $S(a b)=S(b) S(a)^{\mathbf{1}}$.

A *-Hopf algebra is a Hopf algebra with involution $\theta: \mathcal{A} \rightarrow \mathcal{A}$ which satisfies

$$
\begin{aligned}
\theta(\alpha a) & =\alpha^{*} \theta(a), \\
\theta^{2}(a) & =a, \\
\theta(a b) & =\theta(b) \theta(a), \\
\Delta(\theta(a)) & =(\theta \otimes \theta) \Delta(a), \\
\epsilon(\theta(a)) & =\epsilon(a)^{*}, \\
\theta(S(\theta(a))) & =S^{-1}(a),
\end{aligned}
$$

$\alpha \in k$. Here ${ }^{*}$ is the involution on $k$, e.g. complex conjugation when $k=\mathbb{C}$.

A quasitriangular Hopf algebra [8] is a Hopf algebra together with an invertible element $\mathcal{R}=r_{\alpha} \otimes r^{\alpha}$ (summation implied) in $\mathcal{A} \otimes \mathcal{A}$ which must satisfy the relations

$$
\begin{aligned}
(\Delta \otimes \mathrm{id})(\mathcal{R}) & =\mathcal{R}_{13} \mathcal{R}_{23}, \\
(\mathrm{id} \otimes \Delta)(\mathcal{R}) & =\mathcal{R}_{12} \mathcal{R}_{23}, \\
(\tau \circ \Delta)(a) & =\mathcal{R} \Delta(a) \mathcal{R}^{-1},
\end{aligned}
$$

${ }^{1}$ We always make the further assumption that $S$ is bijective, so that the inverse map $S^{-1}$ exists. It too is an antialgebra map. 
where $\tau: \mathcal{A} \otimes \mathcal{A} \rightarrow \mathcal{A} \otimes \mathcal{A}$ is the permutation map $a \otimes b \mapsto b \otimes a$,

$$
\begin{aligned}
& \mathcal{R}_{12}=r_{\alpha} \otimes r^{\alpha} \otimes 1_{\mathcal{A}}, \\
& \mathcal{R}_{13}=r_{\alpha} \otimes 1_{\mathcal{A}} \otimes r^{\alpha}, \\
& \mathcal{R}_{23}=1_{\mathcal{A}} \otimes r_{\alpha} \otimes r^{\alpha},
\end{aligned}
$$

and the multiplication map $m$ has been suppressed on the right-hand side. $\mathcal{R}$ is called the universal $R$-matrix of $\mathcal{A}$, and, as a consequence of these relations, satisfies the quantum Yang-Baxter equation ( $Q Y B E$ )

$$
\mathcal{R}_{12} \mathcal{R}_{13} \mathcal{R}_{23}=\mathcal{R}_{23} \mathcal{R}_{13} \mathcal{R}_{12}
$$

\subsection{Dually Paired Hopf Algebras}

Two *-Hopf algebras $\mathcal{U}$ and $\mathcal{A}$ are said to be dually paired if there exists a nondegenerate inner product $\langle\rangle:, \mathcal{U} \otimes \mathcal{A} \rightarrow k$ such that

$$
\begin{aligned}
\langle x y, a\rangle & =\langle x \otimes y, \Delta(a)\rangle, \\
\left\langle 1_{\mathcal{U}}, a\right\rangle & =\epsilon(a), \\
\langle\Delta(x), a \otimes b\rangle & =\langle x, a b\rangle, \\
\epsilon(x) & =\left\langle x, 1_{\mathcal{A}}\right\rangle, \\
\langle S(x), a\rangle & =\langle x, S(a)\rangle, \\
\langle\theta(x), a\rangle & =\langle x, \theta(S(a))\rangle^{*},
\end{aligned}
$$

where $x, y \in \mathcal{U}$ and $a, b \in \mathcal{A}$. It is easily shown that all the relevant consistency relations between the various operations are satisfied.

Note that the relations above may be used constructively, i.e. given one *-Hopf algebra, one can construct a dually paired *-Hopf algebra; this is the method usually employed when the Drinfel'd double $D(\mathcal{A})$ of a Hopf algebra $\mathcal{A}$ is found [8].

\subsection{Representations of Hopf Algebras and Quan- tum Groups}

Let $\mathcal{U}$ be a Hopf algebra, and suppose $\rho: \mathcal{U} \rightarrow M_{N}(k)$ is a $N \times N$ faithful matrix representation, with entries in $k$, of $\mathcal{U}$. This representation can be used to define 
another Hopf algebra dually paired with $\mathcal{U}$; we take this new Hopf algebra $\mathcal{A}$ to be that which is generated by the $N^{2}$ elements $A^{i}{ }_{j}$ defined by [9]

$$
\rho_{j}^{i}(x) \equiv\left\langle x, A_{j}^{i}\right\rangle
$$

for $x \in \mathcal{U}$. The faithfulness of the representation ensures that this inner product is nondegenerate, and thus the elements of the matrix $A$ are uniquely determined; furthermore, the fact that $\rho$ is an algebra map immediately gives

$$
\Delta\left(A^{i}{ }_{j}\right)=A^{i}{ }_{k} \otimes A^{k}{ }_{j}, \epsilon\left(A_{j}^{i}\right)=\delta_{j}^{i}, S\left(A^{i}{ }_{j}\right)=\left(A^{-1}\right)^{i}{ }_{j} .
$$

The multiplication on $\mathcal{A}$ will of course depend upon the form of the coproduct in $\mathcal{U}$, respectively. However, in the case where $\mathcal{U}$ is quasitriangular with universal $\mathrm{R}$-matrix $\mathcal{R}$, a rather famous result follows; let $x \in \mathcal{U}$, and $\Delta^{\prime}=\tau \circ \Delta$. Using the last of the properties of the universal R-matrix from above, we see

$$
\begin{aligned}
0 \equiv & \left\langle\mathcal{R} \Delta(x)-\Delta^{\prime}(x) \mathcal{R}, A^{i}{ }_{k} \otimes A^{j} \ell_{\ell}\right\rangle \\
= & \left\langle\mathcal{R}, A^{i}{ }_{m} \otimes A^{j}{ }_{n}\right\rangle\left\langle\Delta(x), A^{m}{ }_{k} \otimes A^{n}{ }_{\ell}\right\rangle \\
& -\left\langle\Delta(x), A^{j}{ }_{n} \otimes A^{i}{ }_{m}\right\rangle\left\langle\mathcal{R}, A^{m}{ }_{k} \otimes A^{n}{ }_{\ell}\right\rangle \\
= & \left\langle x, R^{i j}{ }_{m n} A^{m}{ }_{k} A^{n}{ }_{\ell}-A^{j}{ }_{n} A_{m}^{i} R^{m n}{ }_{k \ell}\right\rangle
\end{aligned}
$$

where

$$
R_{k \ell}^{i j}:=\left\langle\mathcal{R}, A^{i}{ }_{k} \otimes A_{\ell}^{j}\right\rangle
$$

is the $N^{2} \times N^{2}$ dimensional numerical $R$-matrix of $\mathcal{A}$. Since $x$ was arbitrary, the vanishing of (2.13) implies that

$$
R A_{1} A_{2}=A_{2} A_{1} R
$$

where the indices have been suppressed, and the subscripts refer to the indices in an obvious way. This is the noted "RAA equation" [4], and gives explicitly the commutation relations between elements of $\mathcal{A}$. It is immediate that the QYBE has the numerical counterpart, simply referred to as the Yang-Baxter equation (YBE):

$$
R_{12} R_{13} R_{23}=R_{23} R_{13} R_{12} .
$$

This leads to the following important definition: a Hopf algebra $\mathcal{A}$ which is dually paired with a quasitriangular Hopf algebra $\mathcal{U}$ is a quantum group [8]. However, we 
often take the opposite view, saying that a quantum group is a Hopf algebra where the $N^{2}$ generators $A^{i}{ }_{j}$ satisfy (2.12) and (2.15), and $R$ satisfies the YBE.

(2.16) was obtained from (2.9) by taking the representation in all three spaces of $\mathcal{U} \otimes \mathcal{U} \otimes \mathcal{U}$, e.g.

$$
(\rho \otimes \rho \otimes \rho) \mathcal{R}_{12} \mathcal{R}_{13} \mathcal{R}_{23}=R_{12} R_{13} R_{23}
$$

It is also useful to consider the case where we take the representation in only one or two of the tensor product spaces. To see this, we define the $N \times N$ matrices $L^{ \pm}$ with entries in $\mathcal{U}$ by

$$
\begin{aligned}
& L^{+}:=(\mathrm{id} \otimes \rho) \mathcal{R} \equiv r_{\alpha} \rho\left(r^{\alpha}\right), \\
& L^{-}:=(\rho \otimes \mathrm{id}) \mathcal{R}^{-1} \equiv \rho\left(S\left(r_{\alpha}\right)\right) r^{\alpha} .
\end{aligned}
$$

From the properties of $\mathcal{R}$, we then find that

$$
\begin{array}{cc}
\Delta\left(L^{ \pm}\right)=L^{ \pm} \dot{\otimes} L^{ \pm}, & \epsilon\left(L^{ \pm}\right)=I, \\
S\left(L^{+}\right)=\left(L^{+}\right)^{-1}=(\mathrm{id} \otimes \rho) \mathcal{R}^{-1}, & S\left(L^{-}\right)=\left(L^{-}\right)^{-1}=(\rho \otimes \mathrm{id}) \mathcal{R}
\end{array}
$$

(where we use the notation $\left.(M \dot{\otimes} N)_{j}^{i}:=M^{i}{ }_{k} \otimes N^{k}{ }_{j}\right)$. Now, suppose we apply id $\otimes \rho \otimes \rho$ to $(2.9)$; the left-hand side is

$$
\begin{aligned}
& \left(\mathrm{id} \otimes \rho_{k}^{i} \otimes \rho_{\ell}^{j}\right) \mathcal{R}_{12} \mathcal{R}_{13} \mathcal{R}_{23}= \\
& \quad\left(\mathrm{id} \otimes \rho_{m}^{i}\right)(\mathcal{R})\left(\mathrm{id} \otimes \rho_{n}^{j}\right)(\mathcal{R})\left(\rho^{m}{ }_{k} \otimes \rho^{n}{ }_{\ell}\right)(\mathcal{R})=\left(L_{1}^{+} L_{2}^{+} R\right)^{i j}{ }_{k \ell} .
\end{aligned}
$$

The right-hand side is computed similarly, and the resulting identity is

$$
L_{1}^{+} L_{2}^{+} R=R L_{2}^{+} L_{1}^{+} \text {. }
$$

By writing (2.9) in various ways using $\mathcal{R}^{-1}$, we find two more independent equations:

$$
L_{1}^{-} L_{2}^{-} R=R L_{2}^{-} L_{1}^{-}, \quad L_{2}^{-} L_{1}^{+} R=R L_{2}^{+} L_{1}^{-} \text {. }
$$

The matrix representations for $L^{ \pm}$are easily found:

$$
\begin{aligned}
\rho_{j}^{i}\left(\left(L^{+}\right)_{\ell}^{k}\right) & =R_{j \ell}^{i k}, \\
\rho_{j}^{i}\left(\left(L^{-}\right)^{k} \ell\right) & =\left(R_{21}^{-1}\right)^{i k}{ }_{j \ell} .
\end{aligned}
$$

As we shall see, these matrices will figure very prominently in the construction of quantum Lie algebras. 
It often becomes convenient to use the permutation matrix $P_{k \ell}^{i j} \equiv \delta_{\ell}^{i} \delta_{k}^{j}$ to define the matrix $\hat{R}$ :

$$
\hat{R}^{i j}{ }_{k \ell}:=(P R)^{i j}{ }_{k \ell} \equiv R^{j i}{ }_{k \ell} \text {. }
$$

$\hat{R}$, not $R$, is the matrix which appears in knot theory; we will not rely upon this interpretation of $\hat{R}$ in this work, although the fact that it satisfies a characteristic ("skein") equation will be used extensively. (See Appendix A.2.2 for more details.)

\subsection{Examples}

\subsubsection{Classical Lie Algebras}

There is a very straightforward way to turn a classical finite-dimensional Lie algebra $\mathbf{g}$ into a quasitriangular Hopf algebra; let $\left\{T_{a} \mid a=1, \ldots, N\right\}$ be a basis for $\mathbf{g}_{;}$and $f_{a b}{ }^{c}$ the structure constants in this basis. Let $\mathcal{A}$ be the universal enveloping algebra of $g$ modulo the commutation relations $\left[T_{a}, T_{b}\right]=T_{a} T_{b}-T_{b} T_{a}=f_{a b}{ }^{c} T_{c}$, denoted $U(\mathbf{g})$. We can then give $\mathcal{A}$ a Hopf algebra structure by defining $\Delta$ and $\epsilon$ to be linear algebra maps and $S$ to be a linear antialgebra map whose actions on the basis elements of $\mathcal{A}$ are given by

$$
\Delta\left(T_{a}\right)=T_{a} \otimes 1_{\mathcal{A}}+1_{\mathcal{A}} \otimes T_{a}, \quad \epsilon\left(T_{a}\right)=0, \quad S\left(T_{a}\right)=-T_{a} .
$$

Furthermore, $\mathcal{A}$ is quite obviously quasitriangular, since $\mathcal{R}=1_{\mathcal{A}} \otimes 1_{\mathcal{A}}$ satisfies all the appropriate relations trivially.

\subsubsection{The Hopf Algebra $U_{q}(s u(2))$}

A nontrivial example of a quasitriangular *-Hopf algebra can be obtained from $s u(2)$; let $\mathcal{A}$ be the universal enveloping algebra of the three generators $H, X_{+}$, and

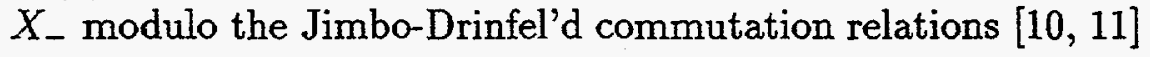

$$
\begin{aligned}
{\left[H, X_{ \pm}\right] } & = \pm 2 X_{ \pm} \\
{\left[X_{+}, X_{-}\right] } & =\frac{q^{H}-q^{-H}}{q-q^{-1}}
\end{aligned}
$$

where $q \in R$. This unital associative algebra is usually denoted by $U_{q}(s u(2))$, the "deformed" universal enveloping algebra of $s u(2)$. The coproducts, counits, 
antipodes and involutions are given by

$$
\begin{array}{rc}
\Delta(H)=H \otimes 1_{\mathcal{A}}+1_{\mathcal{A}} \otimes H, & \Delta\left(X_{ \pm}\right)=X_{ \pm} \otimes q^{\frac{1}{2} H}+q^{-\frac{1}{2} H} \otimes X_{ \pm}, \\
\epsilon(H)=\epsilon\left(X_{ \pm}\right)=0, & \\
S(H)=-H, & S\left(X_{ \pm}\right)=-q^{ \pm 1} X_{ \pm}, \\
\theta(H)=H, & \theta\left(X_{ \pm}\right)=X_{\mp} .
\end{array}
$$

Notice that in the limit $q \rightarrow 1$, we recover the familiar classical $s u(2)$ Hopf algebra described in the previous subsection. The universal R-matrix for $\mathcal{A}$ is given in terms of the above generators, and has the form

$$
\mathcal{R}=\sum_{n=0}^{\infty} \frac{\left(1-q^{-2}\right)^{n}}{[n]_{q} !} q^{\frac{1}{2}\left(H \otimes H+n H \otimes 1_{\mathcal{A}}-n 1_{\mathcal{A}} \otimes H\right)} X_{+}^{n} \otimes X_{-}^{n},
$$

where we use the standard notations for the "quantum number"

$$
[x]_{q}:=\frac{q^{2 x}-1}{q^{2}-1}
$$

and the "quantum factorial"

$$
[n]_{q} !:= \begin{cases}1 & n=0, \\ \prod_{m=1}^{n}[m]_{q} & n=1,2, \ldots\end{cases}
$$

The fundamental repesentations for both the deformed and undeformed cases coincide, i.e. the matrices

$$
H=\left(\begin{array}{cc}
-1 & 0 \\
0 & 1
\end{array}\right), \quad X_{+}=\left(\begin{array}{cc}
0 & 0 \\
-1 & 0
\end{array}\right), \quad X_{-}=\left(\begin{array}{cc}
0 & -1 \\
0 & 0
\end{array}\right),
$$

satisfy the Jimbo-Drinfel'd commutation relations for any value of $q$. When we express the universal R-matrix in this representation, we obtain

$$
R=q^{-\frac{1}{2}}\left(\begin{array}{cccc}
q & 0 & 0 & 0 \\
0 & 1 & 0 & 0 \\
0 & \lambda & 1 & 0 \\
0 & 0 & 0 & q
\end{array}\right),
$$

where $\lambda \equiv q-q^{-1}$. We can also use this representation to find the $2 \times 2$ matrices $L^{ \pm}$, defined in (2.3):

$$
L^{+}=\left(\begin{array}{cc}
q^{-\frac{1}{2} H} & -q^{-\frac{1}{2}} \lambda X_{+} \\
0 & q^{\frac{1}{2} H}
\end{array}\right), \quad L^{-}=\left(\begin{array}{cc}
q^{\frac{1}{2} H} & 0 \\
q^{\frac{1}{2}} \lambda X_{-} & q^{-\frac{1}{2} H}
\end{array}\right) .
$$




\subsubsection{The Quantum Group $G L_{q}(2)$}

The canonical example of a quantum group is the deformed version of $G L(2)$, denoted $G L_{q}(2)$. This is the Hopf algebra generated by the four elements $\{a, b, c, d\}$ satisfying

$$
\begin{gathered}
a b=q b a, \quad a c=q c a, \quad a d-d a=\lambda b c \\
b c=c b, \cdot b d=q d b, \quad c d=q d c .
\end{gathered}
$$

We can express these commutation relations in the form (2.15) by defining

$$
A=\left(\begin{array}{ll}
a & b \\
c & d
\end{array}\right), R=\left(\begin{array}{llll}
q & 0 & 0 & 0 \\
0 & 1 & 0 & 0 \\
0 & \lambda & 1 & 0 \\
0 & 0 & 0 & q
\end{array}\right)
$$

(Note that this differs from the $U_{q}(s u(2))$ R-matrix only by an overall factor of $q^{-\frac{1}{2}}$; we will explain the reason for this in a later section.) For consistency with (2.12), we require

$$
\begin{gathered}
\Delta(a)=a \otimes a+b \otimes c, \quad \Delta(b)=a \otimes b+b \otimes d, \\
\Delta(c)=c \otimes a+d \otimes c, \quad \Delta(d)=c \otimes b+d \otimes d, \\
\epsilon(a)=\epsilon(d)=1, \quad \epsilon(b)=\epsilon(c)=0, \\
S(a)=\left(\operatorname{det}_{q} A\right)^{-1} d, \quad S(b)=-q^{-1}\left(\operatorname{det}_{q} A\right)^{-1} b, \\
S(c)=-q\left(\operatorname{det}_{q} A\right)^{-1} c, \quad S(d)=\left(\operatorname{det}_{q} A\right)^{-1} a,
\end{gathered}
$$

where $\operatorname{det}_{q} A:=a d-q b c$ is the "quantum determinant" of $A$, and is central within the algebra.

\subsection{Sweedler's Notation}

We end this chapter with a discussion of an extremely useful notation which we will use for the remainder of this work. It is referred to as "Sweedler's notation" after the man who first introduced it in [5], and is a way of easing the computations involved in dealing with Hopf algebras.

If $\mathcal{A}$ is a Hopf algebra, then the coproduct $\Delta(a)$ of an element $a \in \mathcal{A}$ will in general consist of a sum of elements in $\mathcal{A} \otimes \mathcal{A}$; the examples presented in the previous 
section show this fact explicitly. Thus, we could in theory write

$$
\Delta(a)=\sum_{i} a_{(1)}^{i} \otimes a_{(2) i}
$$

where $a_{(1)}^{i}$ and $a_{(2) i}$ both live in $\mathcal{A}$. We could easily adopt the standard Einstein summation convention and drop the summation sign, realizing that any pair of identical indices, one up and one down, are to be summed over. However, Sweedler went further than that; he also dropped the indices themselves, preferring to write

$$
\Delta(a)=a_{(1)} \otimes a_{(2)} .
$$

Therefore, anytime an algebra element is subscripted with a number in parentheses, it is understood to be obtained from a coproduct, with the appropriate summation implied. But there's more: using this convention, the coassociativity condition looks like

$$
\left(a_{(1)}\right)_{(1)} \otimes\left(a_{(1)}\right)_{(2)} \otimes a_{(2)}=a_{(1)} \otimes\left(a_{(2)}\right)_{(1)} \otimes\left(a_{(2)}\right)_{(2)} .
$$

Compare this to the case of an associative algebra: the analogous identity to (2.39) is $(a b) c=a(b c)$, and due to this, there is no ambiguity in writing $a b c$. In the coassociative case, we can therefore adopt the unambiguous convention

$$
(\Delta \otimes \mathrm{id}) \Delta(a)=(\mathrm{id} \otimes \Delta) \Delta(a)=a_{(1)} \otimes a_{(2)} \otimes a_{(3)},
$$

again, with the implied sum.

To further illustrate the use of Sweedler's notation, the identity $(\epsilon \otimes \mathrm{id}) \Delta(a)=a$ takes the form

$$
\epsilon\left(a_{(1)}\right) a_{(2)}=a .
$$

Note that the Hopf algebra axioms imply the identity

$$
((m(\mathrm{id} \otimes S) \Delta) \otimes \mathrm{id}) \Delta(a)=a ;
$$

this can be written as

$$
a_{(1)} S\left(a_{(2)}\right) a_{(3)}=a .
$$

For the remainder of this thesis, we will make extensive use of this notation, and the reader is encouraged to familiarize him/herself with its use. 


\section{Chapter 3}

\section{Actions, the Smash Product, and Coactions}

The importance of the three topics in the title above to physics cannot be overstressed, so it is worthwhile to set aside an entire chapter to a discussion of them. We simply rewrite many familiar concepts in the language of Hopf algebras, thus providing a method of generalizing the classical case.

\subsection{Actions and Generalized Derivations}

Suppose we have a unital associative algebra $\mathcal{B}$ and a vector space $\mathcal{V}$; a left action of $\mathcal{B}$ on $\mathcal{V}$ is a bilinear map $\triangleright: \mathcal{B} \otimes \mathcal{V} \rightarrow \mathcal{V}$ satisfying

$$
\begin{aligned}
(x y) \triangleright v & =x \triangleright(y \triangleright v), \\
1_{\mathcal{B}} \triangleright v & =v,
\end{aligned}
$$

for all $x, y \in \mathcal{B}$ and $v \in \mathcal{V}$. (Note that this is merely another way of saying that we have a linear representation of $\mathcal{B}$ with right module $\mathcal{V}$.) A right action $\triangleleft$ of $\mathcal{B}$ on $\mathcal{V}$ can be defined similarly. In the case where $\mathcal{B}$ is a Hopf algebra and $\mathcal{V}$ is a unital algebra, we further require that for $x \in \mathcal{B}$ and $a, b \in \mathcal{V}$,

$$
\begin{aligned}
x \triangleright(a b) & =\left(x_{(1)} \triangleright a\right)\left(x_{(2)} \triangleright b\right) \\
x \triangleright 1_{\mathcal{V}} & =1_{\nu} \epsilon(x) .
\end{aligned}
$$

In this case, $\triangleright$ is called a generalized (left) derivation, and we can interpret $\mathcal{B}$ as an algebra of differential operators which act on functions (i.e. elements of $\mathcal{V}$ ), 
and, as such, may be thought of as providing a means for generalizing infinitesimal transformations. (We will see in a little while that there is a way of generalizing finite transformations as well.)

There are two extremely important examples of such generalized derivations, both of which will be relevant for this work:

- The adjoint action of a Hopf algebra $\mathcal{U}$ on itself is defined as the bilinear map $\stackrel{\text { ad }}{\triangleright} \mathcal{U} \otimes \mathcal{U} \rightarrow \mathcal{U}$ given by $x \otimes y \mapsto x \triangleright y:=x_{(1)} y S\left(x_{(2)}\right)^{1}$; it is a left action as defined above. Similarly, $y \stackrel{\text { ad }}{\triangleleft} x:=S\left(x_{(1)}\right) y x_{(2)}$ defines a perfectly good right action.

- If $\mathcal{U}$ and $\mathcal{A}$ are two dually paired Hopf algebras, we can define the left and right actions of $\mathcal{U}$ on $\mathcal{A}$ respectively as

$$
x \triangleright a:=a_{(1)}\left\langle x, a_{(2)}\right\rangle, \quad a \triangleleft x:=\left\langle x, a_{(1)}\right\rangle a_{(2)} .
$$

As stated above, this allows the interpretation of $\mathcal{U}$ as an algebra of differential operators which act on elements ("functions") of $\mathcal{A}$. (An explicit example of this interpretation is the familiar left action of a quantum mechanical Hamiltonian $H$ on some Schrödinger state $\psi$, namely, $H \triangleright \psi(t)=i \hbar \frac{\partial \psi(t)}{\partial t}$.)

\subsection{The Smash Product}

Let $\mathcal{A}$ and $\mathcal{U}$ be two dually paired Hopf algebras. We can introduce a unital associative algebra which is denoted $\mathcal{A} \rtimes \mathcal{U}$, the "smash product" of $\mathcal{A}$ and $\mathcal{U}$. (This object is also called the "cross product" [7], and is a Hopf algebra generalization of the Heisenberg double and the Weyl semidirect product.) $\mathcal{A} \rtimes \mathcal{U}$ is constructed to be isomorphic to $\mathcal{A} \otimes \mathcal{U}$ as a vector space; this may be seen explicitly through the definition of the multiplication on $\mathcal{A} \rtimes \mathcal{U}$ :

$$
\begin{aligned}
a b & \simeq a b \otimes 1_{\mathcal{U}}, \\
x y & \simeq 1_{\mathcal{A}} \otimes x y, \\
a x & \simeq a \otimes x, \\
x a & \simeq a_{(1)} \otimes x_{(2)}\left\langle x_{(1)}, a_{(2)}\right\rangle,
\end{aligned}
$$

\footnotetext{
${ }^{1}$ Note that if $\mathcal{U}$ is the classical Hopf algebra discussed in Section 2.4.1, the right adjoint action is just the classical commutator: $T_{a} \stackrel{a d}{\triangleright} T_{b}=\left[T_{a}, T_{b}\right]$.
} 
where $a, b \in \mathcal{A}, x, y \in \mathcal{U}$, and the $\simeq$ denotes equivalence under the aforementioned isomorphism. Note that this multiplication is associative, and also that $\mathcal{A} \times \mathcal{U}$ contains subalgebras isomorphic to both $1_{\mathcal{A}} \otimes \mathcal{U}$ and $\mathcal{A} \otimes 1_{\mathcal{U}}$. However, throughout the rest of this work we will be glib and refer to these subalgebras of $\mathcal{A} \rtimes \mathcal{U}$ as $\mathcal{U}$ and $\mathcal{A}$ respectively ${ }^{2}$. With this convention, and the form of the multiplication (3.4), we see that $\mathcal{A} \rtimes \mathcal{U}$ is spanned by elements of the form $a x$ with $a \in \mathcal{A}, x \in \mathcal{U}$, and we can specify all linear maps on $\mathcal{A} \rtimes \mathcal{U}$ by considering their values on such elements.

The physical meaning of the smash product becomes clear when we realize that the multiplication in the smash product $\mathcal{A} \rtimes \mathcal{U}$ may be written as

$$
x a=\left(x_{(1)} \triangleright a\right) x_{(2)},
$$

where the left action is, as it will be for the remainder of this work, the one given in (3.3). Thus, the multiplication relations in $\mathcal{A} \rtimes \mathcal{U}$ may be interpreted as the commutation relations between the differential operators in $\mathcal{U}$ and the elements of $\mathcal{A}$, namely, how to take a differential operator and "move it through" a function. This is a very natural interpretation in physics, and is the one we will adopt.

As an explicit (and important) example of how the smash product works, consider the case where $\mathcal{U}$ is a quasitriangular Hopf algebra, and $\rho$ is a representation of $\mathcal{U}$ which defines the dually paired quantum group $\mathcal{A}$, in the manner of Section 2.3. If $x \in \mathcal{U}$, then the commutation relation between $x$ and a basis element $A^{i}{ }_{j}$ of $\mathcal{A}$ in $\mathcal{A} \rtimes \mathcal{U}$ is

$$
\begin{aligned}
x A_{j}^{i} & =\left(A^{i}{ }_{j}\right)_{(1)}\left\langle x_{(1)},\left(A^{i}{ }_{j}\right)_{(2)}\right\rangle x_{(2)} \\
& =A^{i}{ }_{k}\left\langle x_{(1)}, A^{k}{ }_{j}\right\rangle x_{(2)} \\
& =A^{i}{ }_{k} \rho^{k}{ }_{j}\left(x_{(1)}\right) x_{(2)} .
\end{aligned}
$$

For the case where $x$ is an entry in $L^{ \pm}$, we find

$$
L_{1}^{+} A_{2}=A_{2} R_{21} L_{1}^{+}, \quad L_{1}^{-} A_{2}=A_{2} R^{-1} L_{1}^{-} \text {. }
$$

These relations will come in handy when we discuss quantum Lie algebras.

\footnotetext{
${ }^{2}$ Notice that although $\mathcal{U}$ and $\mathcal{A}$ are both Hopf algebras, $\mathcal{A} \rtimes \mathcal{U}$ is not, i.e. $\mathcal{A} \rtimes \mathcal{U}$ is an algebra that does not admit a Hopf algebra structure (coproduct, counit, antipode) even though the subalgebras $\mathcal{U}$ and $\mathcal{A}$ do.
} 


\subsubsection{Example: The Haar Measure and the Smash Prod- uct}

At this point, it may be instructive to take a slight detour in order to illustrate how the smash product may be used in computations. The example we choose involves the introduction of right-invariant Haar measure on $\mathcal{A}$. This is a linear map $\int: \mathcal{A} \rightarrow k$ which satisfies the two properties

$$
\left(\int a_{(1)}\right) a_{(2)}=\left(\int a\right) 1_{\mathcal{A}}, \quad \int 1_{\mathcal{A}}=1_{k} .
$$

(It is readily shown that these conditions uniquely determine $\int$, and that such a measure is left-invariant as well [12].) We will use the smash product machinery developed above to construct such a measure in the case where $\mathcal{A}$ is finite-dimensional. We introduce the element $E \in \mathcal{A} \rtimes \mathcal{U}$ given by $E:=S^{-1}\left(f^{i}\right) e_{i}$. For $a \in \mathcal{A}$,

$$
\begin{aligned}
E a & =S^{-1}\left(f^{i}\right) e_{i} a \\
& =S^{-1}\left(f^{i}\right) a_{(1)}\left\langle\left(e_{i}\right)_{(1)}, a_{(2)}\right\rangle\left(e_{i}\right)_{(2)} \\
& =S^{-1}\left(f^{i} f^{j}\right) a_{(1)}\left\langle e_{i}, a_{(2)}\right\rangle e_{j} \\
& =S^{-1}\left(f^{j}\right) S^{-1}\left(a_{(2)}\right) a_{(1)} e_{j} \\
& =E \epsilon(a) .
\end{aligned}
$$

and similarly, for $x \in \mathcal{U}$,

$$
x E=\epsilon(x) E .
$$

It is easily shown using these properties that $E^{2}=E$, and therefore

$$
E x a E=\langle x, a\rangle E, \quad E a x E=\epsilon(x) \epsilon(a) E .
$$

We now assume that there exists a Hilbert bimodule of $\mathcal{A} \rtimes \mathcal{U}$ containing the two vacua $\Omega_{\mathcal{U}}$ and $\Omega_{\mathcal{A}}$ which satisfy

$$
\begin{aligned}
x \Omega_{\mathcal{U}}=\Omega_{\mathcal{U}} x & =\epsilon(x) \Omega_{\mathcal{U}}, \\
\Omega_{\mathcal{A}} a=a \Omega_{\mathcal{A}} & =\Omega_{\mathcal{A}} \epsilon(a), \\
\left\langle\Omega_{\mathcal{A}} \mid \Omega_{\mathcal{U}}\right\rangle & =1,
\end{aligned}
$$

for $x \in \mathcal{U}, a \in \mathcal{A}$. (These should recall the definitions of left and right vacua introduced in [13], denoted by $\langle$ and $\rangle$ respectively, which satisfy

$$
\left.\left.\left.L^{+}\right\rangle=L^{-}\right\rangle=I\right\rangle, \quad\langle A=\langle I, \quad\langle\mid\rangle=1 .)
$$


One consequence of these definitions is that $\langle x, a\rangle \equiv\left\langle\Omega_{\mathcal{A}} \mid x a \Omega_{\mathcal{U}}\right\rangle$. We may therefore conclude that $E$ may be represented by $\left|\Omega_{\mathcal{U}}\right\rangle\left\langle\Omega_{\mathcal{A}}\right|$. There also exists an object $\tilde{E} \in \mathcal{A} \rtimes \mathcal{U}$, given by $\tilde{E}=S^{2}\left(e_{i}\right) f^{i}$, which has properties similar to that of $E$, e.g. $\tilde{E}^{2}=\tilde{E}, \tilde{E} x=\tilde{E} \epsilon(x)$ and $a \tilde{E}=\epsilon(a) \tilde{E}$ for $x \in \mathcal{U}, a \in \mathcal{A}$; thus, we represent $\tilde{E}$ by $\left|\Omega_{\mathcal{A}}\right\rangle\left\langle\Omega_{\mathcal{U}}\right|$.

An equivalent way of formulating the second relation of (3.8) is by utilizing the left action (3.3) of $\mathcal{U}$ on $\mathcal{A}$ :

$$
\begin{aligned}
\int x \triangleright a & =\int a_{(1)}\left\langle x, a_{(2)}\right\rangle \\
& =\left\langle x,\left(\int a_{(1)}\right) a_{(2)}\right\rangle \\
& =\left\langle x,\left(\int a\right) 1_{\mathcal{A}}\right\rangle \\
& =\epsilon(x) \int a .
\end{aligned}
$$

Since within $\mathcal{A} \rtimes \mathcal{U}, x \triangleright a \equiv x_{(1)} a S\left(x_{(2)}\right)$, we see that (3.14), together with the uniqueness of $\int$, implies

$$
\int a \equiv \frac{\left\langle\Omega_{\mathcal{U}} \mid a \Omega_{\mathcal{U}}\right\rangle}{\left\langle\Omega_{\mathcal{U}} \mid \Omega_{\mathcal{U}}\right\rangle}
$$

Note, however, that the Hilbert space representations of $E$ and $\tilde{E}$ give

$$
\begin{aligned}
\tilde{E} a E & =\left|\Omega_{\mathcal{A}}\right\rangle\left\langle\Omega_{\mathcal{U}} \mid a \Omega_{\mathcal{U}}\right\rangle\left\langle\Omega_{\mathcal{A}}\right| \\
& =\left|\Omega_{\mathcal{A}}\right\rangle\left(\left\langle\Omega_{\mathcal{U}} \mid \Omega_{\mathcal{U}}\right\rangle \int a\right)\left\langle\Omega_{\mathcal{A}}\right| \\
& =\tilde{E} E \int a .
\end{aligned}
$$

To isolate $\int a$ from this, first we push all the $\mathcal{A}$-elements to the left using (3.4); the result for the left-hand side is

$$
\begin{aligned}
\tilde{E} a E & =S^{2}\left(e_{i}\right) f^{i} a S^{-1}\left(f^{j}\right) e_{j} \\
& =\left(f^{i} a S^{-1}\left(f^{j}\right)\right)_{(1)}\left\langle S^{2}\left(\left(e_{i}\right)_{(1)}\right),\left(f^{i} a S^{-1}\left(f^{j}\right)\right)_{(2)}\right\rangle S^{2}\left(\left(e_{i}\right)_{(2)}\right) e_{j} .
\end{aligned}
$$

$\tilde{E} E$ is obtained by setting $a=1_{\mathcal{A}}$. We may then sandwich this between $\Omega_{\mathcal{U}}$ on the left and $\Omega_{\mathcal{A}}$ on the left to obtain

$$
\left\langle\Omega_{\mathcal{U}} \mid \tilde{E} a E \Omega_{\mathcal{A}}\right\rangle=\left\langle S^{2}\left(e_{i}\right), f^{i} a\right\rangle
$$


Comparing this to (3.16), we see that this is equal to $\left\langle\Omega_{\mathcal{A}} \mid \Omega_{\mathcal{A}}\right\rangle\left\langle\Omega_{\mathcal{U}} \mid \Omega_{\mathcal{U}}\right\rangle \int a$. Therefore, we find an explicit form for the Haar measure on $\mathcal{A}$ :

$$
\int a \equiv \frac{\left\langle S^{2}\left(e_{i}\right), f^{i} a\right\rangle}{\left\langle S^{2}\left(e_{i}\right), f^{i}\right\rangle} .
$$

The finiteness of $\mathcal{A}$ insures that this expression exists. For the case where $\mathcal{A}$ is not finite, the situation is more problematic; the above argument may not hold, because some of the quantities involved, e.g. $\left\langle S^{2}\left(e_{i}\right), f^{i}\right\rangle$, may not exist. Furthermore, it is possible in some cases that we cannot define $\int$ consistently on the entirety of $\mathcal{A}$, and in particular $\int 1_{\mathcal{A}}$ may not exist (this latter case may be a statement of the "noncompactness" of $\mathcal{A}$ ); for both of these possibilities, the computation above may run into problems. In any case, this should serve as an illustration of how the smash product may be used to obtain useful results.

\subsection{Coactions}

Suppose we have a coalgebra $\mathcal{C}$ and a vector space $\mathcal{V}$; a right coaction of $\mathcal{C}$ on $\mathcal{V}$ is a linear map $\Delta_{\mathcal{C}}: \mathcal{V} \rightarrow \mathcal{V} \otimes \mathcal{C}$ satisfying

$$
\begin{aligned}
\left(\Delta_{\mathcal{C}} \otimes \mathrm{id}\right) \Delta_{\mathcal{C}}(v) & =(\mathrm{id} \otimes \Delta) \Delta_{\mathcal{C}}(v), \\
(\mathrm{id} \otimes \epsilon) \Delta_{\mathcal{C}}(v) & =v,
\end{aligned}
$$

for all $v \in \mathcal{V}$, where $\Delta$ and $\epsilon$ are the coproduct and counit on $\mathcal{C}$, respectively. We will often use the Sweedleresque notation $\Delta_{\mathcal{C}}(v)=v^{(1)} \otimes v^{(2)^{\prime}}$, where the unprimed elements live in $\mathcal{V}$, the primed elements in $\mathcal{C}$. The left coaction $c \Delta(v)=v^{(1)^{\prime}} \otimes v^{(2)}$ is defined similarly. If $\mathcal{C}$ and $\mathcal{C}^{\prime}$ are two coalgebras which coact on $\mathcal{V}$ from the left and from the right respectively, we will generally require that they commute, i.e.

$$
(c \Delta \otimes \mathrm{id}) \Delta_{\mathcal{C}^{\prime}}(v)=\left(\mathrm{id} \otimes \Delta_{\mathcal{C}^{\prime}}\right)_{c} \Delta(v)
$$

for $v \in \mathcal{V}$. If $\mathcal{C}$ is a Hopf algebra and $\mathcal{V}$ is a unital algebra, we impose the further conditions that

$$
\begin{aligned}
& \Delta_{\mathcal{C}}(a b)=\Delta_{\mathcal{C}}(a) \Delta_{\mathcal{C}}(b) \\
& \Delta_{\mathcal{C}}\left(1_{\mathcal{V}}\right)=1_{\mathcal{V}} \otimes 1_{\mathcal{C}},
\end{aligned}
$$

for $a, b \in \mathcal{V}$, i.e. $\Delta_{\mathcal{C}}$ must be an algebra homomorphism. If $a \in \mathcal{V}$ satisfies $\Delta_{\mathcal{C}}(a)=$ $a \otimes 1_{\mathcal{C}}$, we say that $a$ is right-invariant (and similarly for left-invariance). 
One coaction which will figure prominently in this work is the adjoint (right) coaction of a Hopf algebra $\mathcal{A}$ on itself. This action $\Delta^{\text {Ad }}: \mathcal{A} \rightarrow \mathcal{A} \otimes \mathcal{A}$ is a right coaction in the first sense above, namely it acts on $\mathcal{A}$ as if it were only a vector space, not a unital algebra. Therefore, it is not a homomorphism, as is easily seen by its definition:

$$
\Delta^{\mathrm{Ad}}(a):=a_{(2)} \otimes S\left(a_{(1)}\right) a_{(3)} .
$$

A comment on terminology: as the reader may have guessed, the reason for the term "coaction" is because of duality. If $\mathcal{B}$ is a unital associative algebra which is dual to a coalgebra $\mathcal{C}$ in the obvious way, and $\triangleright$ is a left action of $\mathcal{B}$ on some vector space $\mathcal{V}$, then there is a natural way to pair it with a right coaction of $\mathcal{C}$ on $\mathcal{V}$ via

$$
v^{(1)}\left\langle x, v^{(2)^{\prime}}\right\rangle=x \triangleright v,
$$

for $x \in \mathcal{B}, v \in \mathcal{V}$. Similarly, a right action will induce a left coaction.

The interpretation of the coaction is straightforward: to illustrate this, let $\mathcal{C}$ be a coalgebra with elements $g_{j}^{i}$ which satisfy $\Delta(g)=g \dot{\otimes} g$ and $\epsilon(g)=I$. Define the right coaction of $\mathcal{C}$ on a basis element $e_{i} \in \mathcal{V}$ via

$$
\Delta_{\mathcal{C}}\left(e_{i}\right):=e_{j} \otimes g^{j}{ }_{i} .
$$

This looks a lot like a simple transformation of the basis elements, which is how we interpret it. If we coact on the first space once more we obtain

$$
\left(\Delta_{\mathcal{C}} \otimes \mathrm{id}\right) \Delta_{\mathcal{C}}\left(e_{i}\right)=e_{k} \otimes g^{k}{ }_{j} \otimes g^{j}{ }_{i},
$$

which is simply two successive "rotations" of the basis. The tensor product between the two indicates that the two transformations are independent of each other. This illustrates the fact that the coaction is the generalization of a finite transformation of an element of $\mathcal{V}$, as opposed to the infinitesimal transformation provided by the action.

\subsection{Actions and Coactions on the Smash Prod- uct}

We have already noted that we can interpret the smash product as the algebra of differential operators and the functions which they act on, with the multiplication within this algebra being interpreted as the commutation relations between the two 
types of elements, i.e. how the differential operators first act on, then are moved through, the functions (3.5). In this section, we discuss how to define actions and coactions on this algebra consistent with this interpretation.

\subsubsection{Bicovariance of the Smash Product}

We now introduce specific actions and coactions in the case where we have the two dually paired Hopf algebras $\mathcal{U}$ and $\mathcal{A}$ and the associated smash product $\mathcal{A} \rtimes \mathcal{U}$. The left and right actions of $\mathcal{U}$ on $\mathcal{A} \times \mathcal{U}$ are defined to be

$$
\begin{aligned}
& x \triangleright \sigma \equiv x_{(1)} \sigma S\left(x_{(2)}\right), \\
& \sigma \triangleleft x \equiv S\left(x_{(1)}\right) \sigma x_{(2)},
\end{aligned}
$$

for $x \in \mathcal{U}, \sigma \in \mathcal{A} \times \mathcal{U}$. Note that for the case where $\sigma \in \mathcal{U}$, these are the left and right adjoint actions, and when $\sigma \in \mathcal{A}$, we reobtain the usual right action of a differential operator $x$ on a function $\sigma$ given by (3.3). Since all elements of $\mathcal{A} \rtimes \mathcal{U}$ have the form $a x, a \in \mathcal{A}$ and $x \in \mathcal{U}$, this gives $\triangleright$ and $\triangleleft$ on all $\mathcal{A} \rtimes \mathcal{U}$.

Keeping in mind that the coaction should describe the transformation properties of the elements of $\mathcal{A} \rtimes \mathcal{U}$, we make the following choices: $\mathcal{A}$ left coacts on $\mathcal{A} \rtimes \mathcal{U}$ so as to leave $\mathcal{U}$ invariant, i.e.

$$
{ }_{\mathcal{A}} \Delta(x) \equiv \mathrm{I}_{\mathcal{A}} \otimes x,
$$

$x \in \mathcal{U}$. Furthermore, $\mathcal{A}$ left and right coacts on $\mathcal{A}$ via the coproduct:

$$
{ }_{\mathcal{A}} \Delta(a)=\Delta_{\mathcal{A}}(a)=\Delta(a),
$$

for $a \in \mathcal{A}$, so on a element $a x \in \mathcal{A} \rtimes \mathcal{U}$,

$$
{ }_{\mathcal{A}} \Delta(a x)=a_{(1)} \otimes a_{(2)} x .
$$

The right coaction of $\mathcal{A}$ on $\mathcal{U}$ is taken to be the natural one induced by the left adjoint action, namely $\Delta_{\mathcal{A}}(x)=x^{(1)} \otimes x^{(2)^{\prime}}$ with

$$
y \stackrel{\text { ad }}{{ }^{\circ}} \equiv x^{(1)}\left\langle y, x^{\left(2^{\prime}\right)}\right\rangle
$$

for $y \in \mathcal{U}$. We can find a more explicit form of $\Delta_{\mathcal{A}}(x)$ by introducing $\left\{e_{i} \mid i \in \mathcal{J}\right\}$ as a basis for $\mathcal{U}(\mathcal{J}$ is the appropriate index set, assumed to be countable), and $\left\{f^{i} \mid i \in \mathcal{J}\right\}$ as the basis for $\mathcal{A}$ such that $\left\langle e_{i}, f^{j}\right\rangle=\delta_{j}^{i}$. We now write $\Delta_{\mathcal{A}}(x)$ as

$$
\Delta_{\mathcal{A}}(x) \equiv x_{i} \otimes f^{i},
$$


where $x_{i} \in \mathcal{U}$. Therefore,

$$
e_{j}^{\mathrm{ad}} \underset{ }{\mathrm{ad}}=x_{i}\left\langle e_{j}, f^{i}\right\rangle=x_{j},
$$

giving

$$
\Delta_{\mathcal{A}}(x)=\left(e_{i}^{\text {ad }} \text { Dx }\right) \otimes f^{i} .
$$

All of the above definitions are consistent with the conditions necessary for $\Delta_{\mathcal{A}}$ to be a right coaction on $\mathcal{A} \rtimes \mathcal{U}$ :

$$
\Delta_{\mathcal{A}}(a x)=a_{(1)}\left(e_{i} \stackrel{\mathrm{ad}}{\triangleright}\right) \otimes a_{(2)} f^{i} ;
$$

As required, the left and right coactions of $\mathcal{A}$ on $\mathcal{A} \rtimes \mathcal{U}$ commute.

Since $\mathcal{A} \times \mathcal{U}$ is an algebra on which $\mathcal{A}$ left and right coacts such that the commutation relations (3.4) transform into themselves, we will often say that $\mathcal{A} \rtimes \mathcal{U}$ is bicovariant, or, more specifically, left-invariant and right-covariant [3].

Going back to the case where $\mathcal{U}$ and $\mathcal{A}$ are a quasitriangular Hopf algebra and its associated quantum group respectively, we see that

$$
{ }_{\mathcal{A}} \Delta(A)=\Delta_{\mathcal{A}}(A)=A \dot{\otimes} A .
$$

The requirement that the coactions respect the commutation relations (3.7) requires that $L^{ \pm}$be left-invariant. Unfortunately, without further information about $\mathcal{U}$, the right coactions cannot be given more explicitly than through (3.34). However, as we will see in Chapter 4, this will not be a major problem.

In a similar fashion to $\Delta_{\mathcal{A}}$, we can define a left coaction of $\mathcal{U}$ on $\mathcal{A} \times \mathcal{U}, u \Delta$ : $\mathcal{A} \rtimes \mathcal{U} \rightarrow \mathcal{U} \otimes \mathcal{A} \rtimes \mathcal{U}$, as $\sigma \mapsto u \Delta(\sigma):=\sigma^{(\overline{1})} \otimes \sigma^{(2)}$. On $\mathcal{U}, u \Delta$ is the coproduct:

$$
u \Delta(x) \equiv x^{(\overline{1})} \otimes x^{(2)}=x_{(1)} \otimes x_{(2)} \equiv \Delta(x) .
$$

On $\mathcal{A}, u \Delta$ is defined again implicitly via

$$
a b=b_{(1)}\left\langle a^{(\overline{1})}, b_{(2)}\right\rangle a^{(2)} .
$$

Using the right action of a function $b$ on another function $a$ given by

$$
a \triangleleft b \equiv S\left(b_{(1)}\right) a b_{(2)},
$$

one can easily show that

$$
u \Delta(a)=e_{i} \otimes\left(a \triangleleft f^{i}\right),
$$

so for $a x \in \mathcal{A} \rtimes \mathcal{U}$,

$$
\mathcal{u} \Delta(a x)=e_{i} x_{(1)} \otimes\left(a \triangleleft f^{i}\right) x_{(2)}
$$




\subsubsection{The Canonical Element of $\mathcal{A} \times \mathcal{U}$}

We are now in a position to introduce the canonical element $C$, which lives in $\mathcal{U} \otimes \mathcal{A}$ :

$$
C:=e_{i} \otimes f^{i} .
$$

$C$ satisfies several relations; for instance, note that

$$
\begin{aligned}
(\Delta \otimes \mathrm{id})(C) & =\Delta\left(e_{i}\right) \otimes f^{i} \\
& =\left(e_{i}\right)_{(1)} \otimes\left(e_{i}\right)_{(2)} \otimes f^{i} \\
& =e_{i} \otimes e_{j} \otimes f^{i} f^{j} \\
& =\left(e_{i} \otimes 1_{\mathcal{U}} \otimes f^{i}\right)\left(1_{\mathcal{U}} \otimes e_{j} \otimes f^{j}\right) \\
& =C_{13} C_{23}
\end{aligned}
$$

(where in going from the second to the third line we have used the duality between $\mathcal{U}$ comultiplication and $\mathcal{A}$-multiplication). Similar calculations also give (id $\otimes \Delta)(C)=$ $C_{12} C_{13}$, as well as the following:

$$
\begin{aligned}
& (S \otimes \mathrm{id})(C)=(\operatorname{id} \otimes S)(C)=C^{-1}, \\
& (\epsilon \otimes \mathrm{id})(C)=(\mathrm{id} \otimes \epsilon)(C)=1_{\mathcal{U}} \otimes 1_{\mathcal{A}} \text {. }
\end{aligned}
$$

So far, $C$ does does not seem to be very interesting; however, to see that it is indeed a useful quantity, we now compute the right coaction of $\mathcal{A}$ on a basis vector in $\mathcal{U}$ : using (3.34),

$$
\begin{aligned}
\Delta_{\mathcal{A}}\left(e_{i}\right) & =\left(e_{j} \underset{\text { ad }}{ } e_{i}\right) \otimes f^{j} \\
& =\left(e_{j}\right)_{(1)} e_{i} S\left(\left(e_{j}\right)_{(2)}\right) \otimes f^{j} \\
& =e_{m} e_{i} S\left(e_{n}\right) \otimes f^{m} f^{n} \\
& =\left(e_{m} \otimes f^{m}\right)\left(e_{i} \otimes 1_{\mathcal{A}}\right)\left(S\left(e_{n}\right) \otimes f^{n}\right) \\
& =C\left(e_{i} \otimes 1_{\mathcal{A}}\right)(S \otimes \mathrm{id})(C),
\end{aligned}
$$

so for any $x \in \mathcal{U}$,

$$
\Delta_{\mathcal{A}}(x)=C\left(x \otimes 1_{\mathcal{A}}\right) C^{-1} .
$$

A similar calculation shows that for $a \in \mathcal{A}$,

$$
\mathcal{u} \Delta(a)=C^{-1}\left(1_{\mathcal{U}} \otimes a\right) C .
$$


So far in this section, we have not made any reference to the smash product; however, when we think of $C$ as living in $(\mathcal{A} \rtimes \mathcal{U}) \otimes(\mathcal{A} \rtimes \mathcal{U})$, with $e_{i}$ and $f^{i}$ as the bases for the subalgebras $\mathcal{U}$ and $\mathcal{A}$ of $\mathcal{A} \times \mathcal{U}$ respectively, $C$ takes on a much expanded role. The first thing we notice is that for $a \in \mathcal{A}$,

$$
\begin{aligned}
C\left(a \otimes 1_{\mathcal{A} \times \mathcal{U}}\right) C^{-1} & =e_{i} a S\left(e_{j}\right) \otimes f^{i} f^{j} \\
& =\left(a_{(1)}\left(e_{i}\right)_{(2)}\left\langle\left(e_{i}\right)_{(1)}, a_{(2)}\right\rangle\right) S\left(e_{j}\right) \otimes f^{i} f^{j} \\
& =a_{(1)}\left\langle\left(e_{k}\right)_{(1)}, a_{(2)}\right\rangle\left(e_{k}\right)_{(2)} S\left(\left(e_{k}\right)_{(3)}\right) \otimes f^{k} \\
& =a_{(1)} \otimes\left\langle e_{k}, a_{(2)}\right\rangle f^{k} \\
& =a_{(1)} \otimes a_{(2)},
\end{aligned}
$$

(where $1_{\mathcal{A} \rtimes \mathcal{U}} \simeq 1_{\mathcal{A}} \otimes 1_{\mathcal{U}}$ is the unit in $\mathcal{A} \rtimes \mathcal{U}$ ) so that

$$
C\left(a \otimes 1_{\mathcal{A} \times u}\right) C^{-1}=\Delta(a) .
$$

Since this is just the right coaction of $\mathcal{A}$ on itself, we can therefore write $\Delta_{\mathcal{A}}$ on all of $\mathcal{A} \rtimes \mathcal{U}$ as

$$
\Delta_{\mathcal{A}}(\sigma)=C\left(\sigma \otimes 1_{\mathcal{A} \times \mathcal{U}}\right) C^{-1}
$$

for any $\sigma \in \mathcal{A} \times \mathcal{U}$. (This expression shows explicitly that $\Delta_{\mathcal{A}}$ is an algebra homomorphism.) We can continue doing calculations along these lines, and we find that for $x \in \mathcal{U}, \Delta(x)=C^{-1}\left(1_{\mathcal{A} \times \mathcal{U}} \otimes x\right) C$, so that the left coaction of $\mathcal{U}$ on $\mathcal{A} \rtimes \mathcal{U}$ is

$$
u \Delta(\sigma)=C^{-1}\left(1_{A \times \mathcal{U}} \otimes \sigma\right) C
$$

for $\sigma \in \mathcal{A} \rtimes \mathcal{U}$. Using these results, together with the coproduct relations for $C$, we obtain the equation

$$
C_{23} C_{12}=C_{12} C_{13} C_{23} .
$$

Alternatively, this equation can be viewed as giving the multiplication on $\mathcal{A} \rtimes \mathcal{U}$ as defined in (3.4).

In the case where $\mathcal{U}$ is a quasitriangular Hopf algebra with universal $\mathrm{R}$-matrix $\mathcal{R}$, the coproduct relations involving $C$ imply the following consistency conditions:

$$
\begin{aligned}
\mathcal{R}_{12} C_{13} C_{23} & =C_{23} C_{13} \mathcal{R}_{12} \\
\mathcal{R}_{23} C_{12} & =C_{12} \mathcal{R}_{13} \mathcal{R}_{23} \\
\mathcal{R}_{13} C_{23} & =C_{23} \mathcal{R}_{13} \mathcal{R}_{12}
\end{aligned}
$$


To see the added significance of these equations, note that

$$
\langle C, a \otimes \mathrm{id}\rangle=a,
$$

where $a \in \mathcal{A}^{3}$. Now, let $\rho: \mathcal{U} \rightarrow M_{N}(k)$ be an $N \times N$ matrix representation of $\mathcal{U}$, and $A$ the matrix of basis elements of $\mathcal{A}$, as in Section 2.3. We see immediately that $(\rho \otimes \mathrm{id})(C)=A$. Now let us apply $\rho^{i}{ }_{k} \otimes \rho_{\ell}{ }_{\ell} \otimes$ id to the first of equations (3.53); the left-hand side gives

$$
\begin{aligned}
\left(\rho_{k}^{i} \otimes \rho_{\ell}^{j} \otimes \mathrm{id}\right) \mathcal{R}_{12} C_{13} C_{23} & =\left(\rho_{m}^{i} \otimes \rho_{n}^{j}\right)(\mathcal{R})\left(\rho^{m}{ }_{k} \otimes \mathrm{id}\right)(C)\left(\rho_{\ell}{ }_{\ell} \otimes \mathrm{id}\right)(C) \\
& =\left(R A_{1} A_{2}\right)^{i j}{ }_{k \ell} .
\end{aligned}
$$

The right-hand side gives $\left(A_{2} A_{1} R\right)^{i j}{ }_{k \ell}$, so we obtain (2.15)! Doing similar gymnastics with the other two equations in (3.53), equations (3.7) can be obtained. Thus, we recover all the commutation relations between the elements of $A$ and between $L^{ \pm}$and $A$.

The physical content of the canonical element formulation presented here has been discussed in [14]: the fact that $C$ generates coactions on $\mathcal{A} \rtimes \mathcal{U}$, i.e. transformations of operators and functions, suggests a possible interpretation of $C$ as a time-evolution operator for certain Hamiltonian systems which may be formulated in a Hopf algebraic manner.

\subsection{Example: The 2-Dimensional Quantum Eu- clidean Group}

In this section, we present an example which will serve to illustrate many of the concepts we discussed in this chapter. We begin by presenting a review of Woronowicz's deformation of the 2-dimensional Euclidean group $E(2)$ [15]: he introduces the *-Hopf algebra $\mathcal{A}$ generated by elements $n, v$, and $\bar{n}$ which satisfy

$$
\begin{array}{rcl}
v n=q n v, & v \bar{n}=q \bar{n} v, & n \bar{n}=\bar{n} n, \\
\Delta(n)=n \otimes v^{-1}+v \otimes n, & \Delta(\bar{n})=\bar{n} \otimes v+v^{-1} \otimes \bar{n}, & \Delta(v)=v \otimes v, \\
\epsilon(n)=\epsilon(\bar{n})=0, & \epsilon(v)=1, & \\
S(n)=-q^{-1} n, & S(\bar{n})=-q \bar{n}, & S(v)=v^{-1}, \\
\theta(n)=\bar{n}, & \theta(v)=v^{-1}, &
\end{array}
$$

\footnotetext{
${ }^{3}$ We use the convention that the inner product of any object with the identity map returns that object, e.g. $\langle x, \mathrm{id}\rangle=x$.
} 
with $q \in \mathbf{R}$. For the calculations which follow, it is convenient to introduce the elements $\gamma, m$ and $\bar{m}$, defined by

$$
\gamma:=-2 i \ln v, \quad m:=n v, \quad \bar{m}:=v^{-1} \bar{n},
$$

All of these new elements have vanishing counit, and have commutation relations, coproducts, antipodes, and conjugates given by

$$
\begin{array}{rcc}
{[\gamma, m]=-2 i \ln q m,} & {[\gamma, \bar{m}]=-2 i \ln q \bar{m},} & m \bar{m}=q^{2} \bar{m} m, \\
\Delta(m)=m \otimes 1_{\mathcal{A}}+e^{i \gamma} \otimes m, & \Delta(\bar{m})=\bar{m} \otimes 1_{\mathcal{A}}+e^{-i \gamma} \otimes \bar{m}, & \\
\Delta(\gamma)=\gamma \otimes 1_{\mathcal{A}}+1_{\mathcal{A}} \otimes \gamma, & \\
S(m)=-e^{-i \gamma} m, & S(\bar{m})=-e^{i \gamma} \bar{m}, & S(\gamma)=-\gamma, \\
\theta(m)=\bar{m}, & \theta(\gamma)=\gamma . &
\end{array}
$$

Note that the $2 \times 2$ matrices $E$ and $\bar{E}=\theta(E)$ given by

$$
E=\left(\begin{array}{cc}
e^{i \gamma} & m \\
0 & 1_{\mathcal{A}}
\end{array}\right), \quad \bar{E}=\left(\begin{array}{cc}
e^{-i \gamma} & \bar{m} \\
0 & 1_{\mathcal{A}}
\end{array}\right)
$$

satisfy the relations (2.12). These are exactly the relations one would expect for a quantum group matrix, despite the fact that we have not seen any sign of an $R$ matrix yet. To further interpret what we have here, let us introduce the deformed complex plane $C_{q}$ as the unital associative algebra generated by $z, \bar{z}$ which satisfy $z \bar{z}=q^{2} \bar{z} z$. We define a left coaction of $\mathcal{A}$ on $\mathrm{C}_{q}$ as

$$
{ }_{\mathcal{A}} \Delta(z):=e^{i \gamma} \otimes z+m \otimes 1_{\mathcal{C}_{q}},{ }_{A} \Delta(\bar{z}):=e^{-i \gamma} \otimes \bar{z}+\bar{m} \otimes 1_{\mathfrak{C}_{q}}
$$

By introducing the column vectors $z^{i}:=\left(\begin{array}{c}z \\ 1_{\mathbb{C}_{q}}\end{array}\right)$ and $\bar{z}^{i}:=\left(\begin{array}{c}\bar{z} \\ 1_{\mathbb{C}_{q}}\end{array}\right)$, these can be rewritten as

$$
{ }_{\mathcal{A}} \Delta\left(z^{i}\right)=E^{i}{ }_{j} \otimes z^{j},{ }_{\mathcal{A}} \Delta\left(\bar{z}^{i}\right)=\bar{E}^{i}{ }_{j} \otimes \bar{z}^{j}
$$

This suggests identifying $\mathcal{A}$ as a deformation of the 2-dimensional Euclidean group, which we denote $E_{q}(2)$ (this is just a particular example of an inhomogeneous quantum group $[16,17]$ ). We will now make an explicit construction (following the methods in [18]) of the dually paired *-Hopf algebra $\mathcal{U}$, which will be identifiable with $U_{q}(e(2))$, the quantized universal enveloping algebra of the 2-dimensional Euclidean 
algebra. We choose $\operatorname{span}\left\{\gamma^{a} m^{b} \bar{m}^{c} \mid a, b, c=0,1, \ldots\right\}$ as a basis for $\mathcal{A}$, and define $h$, $\mu$, and $\nu$ as the elements of $\mathcal{U}$ whose inner products with these basis elements are

$$
\begin{aligned}
\left\langle\mu, \gamma^{a} m^{b} \bar{m}^{c}\right\rangle: & =\delta_{a, 0} \delta_{b, 1} \delta_{c, 0}, \quad\left\langle\nu, \gamma^{a} m^{b} \bar{m}^{c}\right\rangle:=\delta_{a, 0} \delta_{b, 0} \delta_{c, 1}, \\
\left\langle h, \gamma^{a} m^{b} \bar{m}^{c}\right\rangle: & =\delta_{a, 1} \delta_{b, 0} \delta_{c, 0} .
\end{aligned}
$$

We require the two algebras to be dually paired; therefore, using the coproduct on $\mathcal{A}$ to obtain the multiplication on $\mathcal{U}$ gives

$$
\left\langle\nu^{k} \mu^{\ell} h^{n}, \gamma^{a} m^{b} \bar{m}^{c}\right\rangle=[k]_{q} ![\ell]_{q^{-1}} ! n ! \delta_{n, a} \delta_{\ell, b} \delta_{k, c}
$$

so $\left\{\nu^{k} \mu^{\ell} h^{n} \mid k, \ell, n=0,1, \ldots\right\}$ is a basis for $\mathcal{U}$. The rest of the *-Hopf algebra structure of $\mathcal{U}$ can be similarly obtained:

$$
\begin{array}{rcc}
{[h, \mu]=i \mu,} & {[h, \nu]=-i \nu,} & \mu \nu=q^{2} \nu \mu, \\
\Delta(\mu)=\mu \otimes q^{2 i h}+1_{\mathcal{U}} \otimes \mu, & \Delta(\nu)=\nu \otimes q^{2 i h}+1_{\mathcal{U}} \otimes \nu, & \\
\Delta(h)=h \otimes 1_{\mathcal{U}}+1_{\mathcal{U}} \otimes h, & \epsilon(\mu)=\epsilon(\nu)=\epsilon(h)=0, & \\
S(\mu)=-\mu q^{-2 i h}, & S(\nu)=-\nu q^{-2 i h}, & S(h)=-h, \\
\theta(h)=-h, & \theta(\mu)=-q^{2} \nu, & \theta(\nu)=-q^{-2} \mu .
\end{array}
$$

Defining new operators $J, P_{ \pm}$as

$$
J:=i h, \quad P_{+}:=q^{1-i h} \nu, \quad P_{-}:=-\mu q^{-1-i h},
$$

we find that they all have vanishing counit, $\theta(J)=J, \theta\left(P_{ \pm}\right)=P_{\mp}$, and

$$
\begin{array}{rc}
{\left[J, P_{ \pm}\right]= \pm P_{ \pm},} & {\left[P_{+}, P_{-}\right]=0,} \\
\Delta\left(P_{ \pm}\right)=P_{ \pm} \otimes q^{J}+q^{-J} \otimes P_{ \pm}, & \Delta(J)=J \otimes 1_{\mathcal{U}}+1_{\mathcal{U}} \otimes J, \\
S(J)=-J, & S\left(P_{ \pm}\right)=-q^{ \pm 1} P_{ \pm} .
\end{array}
$$

An interesting fact is that as a unital associative *-algebra, this is the undeformed UEA of the classical algebra $e(2)$ [19]. However, it has a nontrivial *-Hopf algebra structure.

The inner products between these new generators of $\mathcal{U}$ and the basis elements of $\mathcal{A}$ can be computed; they are

$$
\begin{aligned}
\left\langle P_{+}^{k} P_{-}^{\ell} J^{n}, \gamma^{a} m^{b} \bar{m}^{c}\right\rangle & =\frac{i^{a} a !(-1)^{\ell+a-n}}{(a-n) !} q^{-\frac{1}{2}(k-\ell)(k+\ell-1)+\ell(k-1)}\left(\ln q^{k+\ell}\right)^{a-n} \\
& \times[k]_{q} ![\ell]_{q^{-1}} ! \Theta_{a, n} \delta_{\ell, b} \delta_{k, c}
\end{aligned}
$$


where $\Theta_{i, j}$ is 1 if $i \geq j$ and zero otherwise. With these inner products in hand, plus the coproducts given in (3.66), we may use (3.3) to find the actions of $J$ and $P_{ \pm}$on the basis elements of $\mathcal{A}$. They are

$$
\begin{aligned}
P_{+} \triangleright \gamma^{a} m^{b} \bar{m}^{c} & =q^{2 b}[c]_{q} e^{-i \gamma}(\gamma-i \ln q)^{a} m^{b} \bar{m}^{c-1} \\
P_{-} \triangleright \gamma^{a} m^{b} \bar{m}^{c} & =-\frac{1}{q}[b]_{q^{-1}} e^{i \gamma}(\gamma-i \ln q)^{a} m^{b-1} \bar{m}^{c} \\
J \triangleright \gamma^{a} m^{b} \bar{m}^{c} & =i a \gamma^{a-1} m^{b} \bar{m}^{c} .
\end{aligned}
$$

Therefore, if $f(\gamma, m, \bar{m})$ is a function written in terms of the basis elements of $\mathcal{A}$, we find

$$
\begin{aligned}
P_{+} \triangleright f(\gamma, m, \bar{m}) & =e^{-i \gamma} \frac{f\left(\gamma-i \ln q, q^{2} m, q^{2} \bar{m}\right)-f\left(\gamma-i \ln q, q^{2} m, \bar{m}\right)}{q^{2}-1} \frac{1}{\bar{m}} \\
P_{+} \triangleright f(\gamma, m, \bar{M}) & =-\frac{1}{q} e^{i \gamma} \frac{f\left(\gamma-i \ln q, q^{-2} m, q^{-2} \bar{m}\right)-f\left(\gamma-i \ln q, m, q^{-2} \bar{m}\right)}{q^{-2}-1} \frac{1}{m} \\
J_{\triangleright} f(\gamma, m, \bar{m}) & =i \frac{\partial}{\partial \gamma} f(\gamma, m, \bar{m}) .
\end{aligned}
$$

In the $q \rightarrow 1$ limit, we see that $\left(P_{+} \triangleright\right) \rightarrow e^{-i \gamma} \frac{\partial}{\partial \bar{m}},\left(P_{-} \triangleright\right) \rightarrow-e^{i \gamma} \frac{\partial}{\partial m}$, and $\left(J_{D}\right) \rightarrow i \frac{\partial}{\partial \gamma}$, which are precisely what we'd expect. However, for the $q \neq 1$ case, the actions of $P_{ \pm}$give differences rather than derivatives. It might therefore be possible to use these expressions to consistently regularize a 2-dimensional theory with Euclidean symmetry: the expressions for $P_{ \pm} \triangleright$ above involve differences between neighboring points on a 3-dimensional lattice where the $\gamma$-lattice spacing is $i \ln q$, and neighboring points in the $m$ - and $\bar{m}$-lattices differ by a factor of $q^{ \pm 2}$ (the action of $J$ can be treated classically). In the $q \rightarrow 1$ limit, the lattice spacings will shrink to zero, but the finiteness of the theory for $q \neq 1$ may allow us to control any divergences which arise.

What are the commutation relations within the smash product for this example? Since we have the inner products and coproducts, we just turn the crank and use (3.4) to find

$$
\begin{array}{ll}
{\left[P_{ \pm}, \gamma\right]=-i \ln q P_{ \pm},} & {[J, \gamma]=i} \\
{\left[P_{+}, \bar{m}\right]=e^{-i \gamma} q^{J},} & {\left[P_{-}, m\right]=-\frac{1}{q} e^{i \gamma} q^{J},}
\end{array}
$$

with all other commutators between $J, P_{+}, P_{-}$and $\gamma, m, \bar{m}$ vanishing. All commutation relations within $\mathcal{A} \rtimes \mathcal{U}$ may be obtained from these. 


\section{Chapter 4}

\section{Quantum Lie Algebras}

\subsection{Basics of Quantum Lie Algebras}

Let $\mathcal{U}$ be a Hopf algebra; we say that $\mathcal{U}$ is a quantum Lie algebra (QLA) iff there exists a finite subspace $\mathrm{g} \subset \mathcal{U}(\operatorname{dim} \mathrm{g}=n)$ which has the following properties:

1. As a vector space, $\mathcal{U} \equiv U_{q}(\mathrm{~g})$, i.e. the universal enveloping algebra (UEA) of $\mathrm{g}$ modulo commutation relations;

2. The adjoint action $\stackrel{\text { ad }}{\triangleright}$ closes on $\mathbf{g}$, i.e. $y \triangleright$ ad $\chi \in \mathbf{g}$ for all $y \in \mathcal{U}$ and $\chi \in \mathbf{g}$;

3. $\Delta(\chi) \in \mathcal{U} \otimes\left(1_{\mathcal{U}} \oplus \mathrm{g}\right)$ for all $\chi \in \mathrm{g}$;

4. For all $\chi \in \mathrm{g}, \epsilon(\chi)=0$.

(the $q$ subscript in (1) simply indicates that the commutation relations may be deformed relative to the classical case.) Let $\left\{\chi_{A} \mid A=1,2, \ldots, n\right\}$ be a basis for $\mathbf{g}$ [20]; (3) therefore requires the coproduct to take the form

$$
\Delta\left(\chi_{A}\right)=\chi_{A}^{\prime} \otimes 1_{\mathcal{U}}+\mathcal{O}_{A}^{B} \otimes \chi_{B},
$$

where $\chi_{A}^{\prime}, \mathcal{O}_{A}^{B} \in \mathcal{U}$. However, the fact that $\mathcal{U}$ is a Hopf algebra requires

$$
\begin{aligned}
\chi_{A} & \equiv(\mathrm{id} \otimes \epsilon) \Delta\left(\chi_{A}\right) \\
& =\chi_{A}^{\prime} \epsilon\left(1_{\mathcal{U}}\right)+\mathcal{O}_{A}{ }^{B} \epsilon\left(\chi_{B}\right),
\end{aligned}
$$

so (4) imposes the condition that

$$
\Delta\left(\chi_{A}\right)=\chi_{A} \otimes 1_{\mathcal{U}}+\mathcal{O}_{A}^{B} \otimes \chi_{B}
$$


Using this, and the other requirements for $\mathcal{U}$ to be a Hopf algebra, we find the following:

$$
\begin{array}{r}
\Delta\left(\mathcal{O}_{A}^{B}\right)=\mathcal{O}_{A}{ }^{C} \otimes \mathcal{O}_{C}{ }^{B} ; \\
\left.S\left(\mathcal{O}_{A}^{B}\right)=\left(\mathcal{O}^{-1}\right)_{A}{ }^{B}, \quad S\left(\chi_{A}\right)=-S\left(\mathcal{O}_{A}^{B}\right)=\delta_{A}^{B}\right) \\
\chi_{B}
\end{array}
$$

Condition (2) allows us express the commutation relations between elements of $\mathbf{g}$ (and therefore between all elements of $\mathcal{U}$ ) in a more transparent form. To see this, we define the $k$-numbers $\hat{\mathbf{R}}^{A B} C D$ and $f_{A B}{ }^{C}$ via

$$
\begin{aligned}
\chi_{A}^{\mathrm{ad}} \chi_{B} & :=f_{A B}^{C} \chi_{C}, \\
\mathcal{O}_{C}{ }^{B^{\mathrm{ad}}} \stackrel{\chi_{D}}{ } & :=\hat{\mathrm{R}}^{A B}{ }_{C D} \chi_{A} .
\end{aligned}
$$

$\hat{\mathbf{R}}$ is referred to as the $\mathbf{R}$-matrix of $\mathbf{g}$, and the $f \mathrm{~s}$ are, as in the classical case, just the structure constants of $\mathbf{g}$. $\hat{\mathbf{R}}$ is invertible, with $\hat{\mathbf{R}}^{-1}$ given by

$$
S^{-1}\left(\mathcal{O}_{D}{ }^{A}\right) \stackrel{\text { ad }}{\triangleright} \chi_{C}=\left(\hat{\mathbf{R}}^{-1}\right)^{A B}{ }_{C D} \chi_{B},
$$

and the matrix $\tilde{\mathbf{R}}$ (see Appendix A) is given by

$$
S\left(\mathcal{O}_{C}{ }^{A}\right) \stackrel{\text { ad }}{\triangleright} \chi_{D}=\tilde{\mathbf{R}}^{A B}{ }_{C D} \chi_{B} .
$$

Now, note that for any Hopf algebra $\mathcal{U}$, we have the following identity:

$$
\left(x_{(1)} \stackrel{\text { ad }}{\triangleright}\right) x_{(2)}=x_{(1)} y S\left(x_{(2)}\right) x_{(3)}=x y
$$

for all $x, y \in \mathcal{U}$. Therefore, using (4.3),

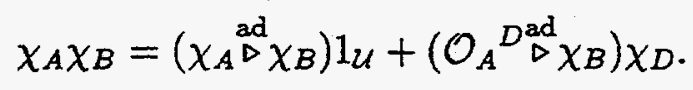

When we use the explicit forms of the adjoint actions, we have

$$
\chi_{A} \chi_{B}-\hat{\mathrm{R}}^{C D}{ }_{A B} \chi_{C} \chi_{D}=f_{A B}{ }^{C} \chi_{C},
$$

which are the commutation relations between basis elements of $g$. Here we see explicitly the "deformation" of the algebra, via the R-matrix. In the classical case, $\hat{\mathrm{R}}^{A B}{ }_{C D}=\delta_{D}^{A} \delta_{C}^{B}$, and the left-hand side of (4.10) reduces to the commutator. Thus, the commutation relations between the generators are parametrized not only by 
the structure constants, but by the R-matrix as well. (However, this is not the full story; in the next section, we will also see that there will have to be certain numerical conditions between $\hat{\mathbf{R}}$ and the $f$ s to ensure consistency of the algebra.) The adjoint action is still given entirely by the structure constants, though.

By continuing along the above lines, we find more commutation relations, involving the $\mathcal{O}_{\mathrm{s}}$ :

$$
\begin{aligned}
\hat{\mathbf{R}}^{E F}{ }_{A B} \mathcal{O}_{E}{ }^{C} \mathcal{O}_{F}{ }^{D} & =\mathcal{O}_{A}^{E} \mathcal{O}_{B}{ }^{F} \hat{\mathbf{R}}^{C D}{ }_{E F} \\
\chi_{A} \mathcal{O}_{B}{ }^{C}-\hat{\mathbf{R}}^{D E}{ }_{A B} \mathcal{O}_{D}{ }^{C} \chi_{E} & =f_{A B}{ }^{D} \mathcal{O}_{D}{ }^{C}-\mathcal{O}_{A}{ }^{D} \mathcal{O}_{B}{ }^{E} f_{D E}{ }^{C} \\
\mathcal{O}_{A}{ }^{B} \chi_{C} & =\hat{\mathbf{R}}^{D E}{ }_{A C} \chi_{D} \mathcal{O}_{E}{ }^{B}
\end{aligned}
$$

The last of these is a consistency condition the fact that the elements of $\mathcal{O}_{A}{ }^{B}$ are expressible in terms of the $\chi \mathrm{s}$, due to $\mathcal{U}=U_{q}(\mathrm{~g})$. We also find that the selfconsistency of these relations requires

$$
\hat{\mathbf{R}}_{12} \hat{\mathbf{R}}_{23} \hat{\mathbf{R}}_{12}=\hat{\mathbf{R}}_{23} \hat{\mathbf{R}}_{12} \hat{\mathbf{R}}_{23} \text {. }
$$

So even though we have not said anything at all about the quasitriangularity of $\mathcal{U}$, we see that the R-matrix associated with a QLA must satisfy a numerical YangBaxter equation. However, as we will see, this matrix is not the representation of the universal $R$-matrix when $\mathcal{U}$ is in fact quasitriangular.

\subsection{The Adjoint Representation}

The closure of $\mathrm{g}$ under the adjoint action defines the adjoint representation ad of $\mathcal{U}$ (with module $\mathrm{g}$ ) as

$$
y \stackrel{\mathrm{ad}}{\triangleright \chi_{A}}=\chi_{B}(\operatorname{ad}(y))_{A}^{B} .
$$

As is discussed in Chapter 2, this motivates the introduction of elements $A^{A}{ }_{B}$ in the Hopf algebra $\mathcal{A}$ dually paired with $\mathcal{U}$, given by

$$
\operatorname{ad}(y)_{B}^{A}=\left\langle y, A_{B}^{A}\right\rangle \text {. }
$$

Therefore, we find

$$
\begin{array}{r}
\left\langle\chi_{A}, \mathrm{~A}^{C}{ }_{B}\right\rangle=f_{A B}{ }^{C}, \quad\left\langle\mathcal{O}_{C}^{B}, A^{A} D=\hat{\mathbf{R}}^{A B}{ }_{C D}\right. \\
\left\langle S^{-1}\left(\mathcal{O}_{D}{ }^{A}\right), \mathrm{A}_{C}^{B}\right\rangle=\left(\hat{\mathbf{R}}^{-1}\right)_{C D}^{A B}, \quad\left\langle S\left(\mathcal{O}_{C}{ }^{A}\right), \mathrm{A}^{B}{ }_{D}\right\rangle=\tilde{\mathbf{R}}_{C D}^{A B}
\end{array}
$$


By using the definition of the right coaction of $\mathcal{A}$ on $\mathcal{U}$ given in Chapter 3 , we see that

$$
\Delta_{\mathcal{A}}\left(\chi_{A}\right)=\chi_{B} \otimes A^{B}{ }_{A} .
$$

For consistency with the defining properties of the QLA, the adjoint matrices $A$ must satisfy the following:

$$
\begin{gathered}
\Delta\left(\mathrm{A}^{A}{ }_{B}\right)=\mathrm{A}^{A}{ }_{C} \otimes \mathrm{A}^{C}{ }_{B}, \quad \epsilon\left(\mathrm{A}^{A}{ }_{B}\right)=\delta_{B}^{A}, \\
S\left(\mathrm{~A}^{A}{ }_{B}\right)=\left(\mathrm{A}^{-1}\right)^{A}{ }_{B}, \hat{\mathrm{R}} \mathrm{A}_{1} \mathrm{~A}_{2}=\mathrm{A}_{1} \mathrm{~A}_{2} \hat{\mathrm{R}}, \\
f_{A B}{ }^{D} \mathrm{~A}^{C}{ }_{D}=\mathrm{A}^{D}{ }_{A} \mathrm{~A}^{E_{B}}{ }_{B} f_{D E}{ }^{C} .
\end{gathered}
$$

Once again, even though we did not assume that $\mathcal{U}$ was quasitriangular, the dual appearing here has a very quantum-grouplike structure to it.

We can use the above properties of $A$ to find several numerical relations among the R-matrix and structure constants; for instance, if we take the inner product of $\mathrm{A}^{M}{ }_{N}$ and (4.10), we find the deformed version of the Jacobi identity:

$$
f_{A L}{ }^{M} f_{B N}{ }^{L}-\hat{\mathbf{R}}^{C D}{ }_{A B} f_{C L}{ }^{M} f_{D N}{ }^{L}=f_{A B}{ }^{C} f_{C N}{ }^{M} .
$$

Repeating this for the first of (4.11) just recovers the numerical Yang-Baxter relation for $\hat{\mathbf{R}}$; the other two give

$$
\begin{aligned}
\hat{\mathbf{R}}^{D C}{ }_{B N} f_{A D}{ }^{M}-\hat{\mathbf{R}}^{D E}{ }_{A B} \hat{\mathbf{R}}^{M C}{ }_{D F} f_{E N} F & =\hat{\mathbf{R}}^{M C}{ }_{D N} f_{A B}{ }^{D}-\hat{\mathrm{R}}^{D F}{ }_{B N} \hat{\mathrm{R}}^{M E}{ }_{A D} f_{E F}{ }^{C} \\
\hat{\mathbf{R}}^{M B}{ }_{A D} f_{C N} D & =\hat{\mathbf{R}}^{D E}{ }_{A C} \hat{\mathbf{R}}^{F B}{ }_{E N} f_{D F}{ }^{M}
\end{aligned}
$$

These are the numerical relations alluded to earlier which to specify the QLA.

The commutation relations between the elements of $\mathcal{U}$ and the adjoint matrices can be determined by using the inner products given above and the smash product. They take the form

$$
\begin{aligned}
\chi_{A} A^{B}{ }_{C} & =\hat{\mathrm{R}}^{D E}{ }_{A C} \mathrm{~A}^{B}{ }_{D} \chi_{E}+f_{A C}{ }^{D} \mathrm{~A}^{B}{ }_{D}, \\
\mathcal{O}_{A}{ }^{B} \mathrm{~A}^{C}{ }_{D} & =\hat{\mathrm{R}}^{E F}{ }_{A D} \mathrm{~A}^{C}{ }_{E} \mathcal{O}_{F}{ }^{B} .
\end{aligned}
$$

\subsection{Quasitriangular Quantum Lie Algebras}

Now, in the case where $\mathcal{U}$ is in fact quasitriangular, we can use the contents of Chapter 2.3 to immediately obtain a QLA [21]. This is done as follows: let $\rho$ be 
a representation of $\mathcal{U}$; we therefore have the matrices $L^{ \pm}$which satisfy $(2.21)$ and (2.22). We define the matrix $Y$ by $[22,13]$

$$
Y:=L^{+} S\left(L^{-}\right) \equiv(\rho \otimes \mathrm{id}) \mathcal{R}_{21} \mathcal{R}
$$

this matrix therefore satisfies

$$
\begin{aligned}
& L_{1}^{+} Y_{2}=R_{21}^{-1} Y_{2} R_{21} L_{1}^{+}, \quad L_{1}^{-} Y_{2}=R Y_{2} R^{-1} L_{1}^{-} \\
& R_{21} Y_{1} R Y_{2}=Y_{2} R_{21} Y_{1} R .
\end{aligned}
$$

$Y$ has coproduct, counit and antipode given by

$$
\begin{aligned}
& \Delta\left(Y^{i}{ }_{j}\right)=\left(L^{+}\right)^{i}{ }_{k} S\left(\left(L^{-}\right)^{\ell}{ }_{j}\right) \otimes Y^{k}{ }_{\ell}, \quad \epsilon\left(Y^{i}{ }_{j}\right)=\delta_{j}^{i}, \\
& S\left(Y^{i}{ }_{j}\right)=S^{2}\left(\left(L^{-}\right)^{k}{ }_{j}\right) S\left(\left(L^{+}\right)^{i}{ }_{k}\right) .
\end{aligned}
$$

We would naturally like to know what the coactions of $\mathcal{A}$ are on $Y$; since $L^{ \pm}$are left-invariant, so is $Y$. The right coaction is a bit more problematic, since we do not have explicit forms for the right coactions of $L^{ \pm}$. However, we can get around this in the following way: for $a \in \mathcal{A}$, we define $\Upsilon_{a} \in \mathcal{U}$ as

$$
\Upsilon_{a}:=\left\langle\mathcal{R}_{21} \mathcal{R}, a \otimes \mathrm{id}\right\rangle
$$

Thus, by definition,

$$
Y_{j}^{i}=\Upsilon_{A^{i}{ }_{j}}
$$

Now, we note that for $x \in \mathcal{U}$,

$$
\begin{aligned}
\underset{x \triangleright \Upsilon_{a}}{\mathrm{ad}} & =x \stackrel{\mathrm{ad}}{\Upsilon}\left(\mathcal{R}_{21} \mathcal{R}, a \otimes \mathrm{id}\right\rangle \\
& =\left\langle\left(1_{\mathcal{U}} \otimes x_{(1)}\right) \mathcal{R}_{21} \mathcal{R}\left(1_{\mathcal{U}} \otimes S\left(x_{(2)}\right)\right), a \otimes \mathrm{id}\right\rangle \\
& =\left\langle\left(1_{\mathcal{U}} \otimes x_{(1)}\right) \mathcal{R}_{21} \mathcal{R}\left(S\left(x_{(3)}\right) x_{(4)} \otimes S\left(x_{(2)}\right)\right), a \otimes \mathrm{id}\right\rangle \\
& =\left\langle\left(1_{\mathcal{U}} \otimes x_{(1)}\right) \mathcal{R}_{21} \mathcal{R} \Delta\left(S\left(x_{(2)}\right)\right)\left(x_{(3)} \otimes 1_{\mathcal{U}}\right), a \otimes \mathrm{id}\right\rangle \\
& =\left\langle\left(1_{\mathcal{U}} \otimes x_{(1)}\right) \Delta\left(S\left(x_{(2)}\right)\right) \mathcal{R}_{21} \mathcal{R}\left(x_{(3)} \otimes 1_{\mathcal{U}}\right), a \otimes \mathrm{id}\right\rangle \\
& =\left\langle\left(S\left(x_{(3)}\right) \otimes x_{(1)} S\left(x_{(2)}\right)\right) \mathcal{R}_{21} \mathcal{R}\left(x_{(4)} \otimes 1_{\mathcal{U}}\right), a \otimes \mathrm{id}\right\rangle \\
& =\left\langle\left(S\left(x_{(1)}\right) \otimes 1_{\mathcal{U}}\right) \mathcal{R}_{21} \mathcal{R}\left(x_{(2)} \otimes 1_{\mathcal{U}}\right), a \otimes \mathrm{id}\right\rangle \\
& =\left\langle S\left(x_{(1)}\right) \otimes x_{(2)}, a_{(1)} \otimes a_{(3)}\right\rangle\left\langle\mathcal{R}_{21} \mathcal{R}, a_{(2)} \otimes \mathrm{id}\right\rangle \\
& =\left\langle x, S\left(a_{(1)}\right) a_{(3)}\right\rangle \Upsilon_{a_{(2)}},
\end{aligned}
$$


where we have made ample use of the various properties of dually paired Hopf algebras, and used the very important fact that $\mathcal{R}_{21} \mathcal{R}$ commutes with all of $\Delta(\mathcal{U})$. Thus, from (3.34),

$$
\begin{aligned}
\Delta_{\mathcal{A}}\left(\Upsilon_{a}\right) & =\left(e_{i}^{\text {ad }} \Upsilon_{a}\right) \otimes f^{i} \\
& =\left\langle e_{i}, S\left(a_{(1)}\right) a_{(3)}\right\rangle \Upsilon_{a_{(2)}} \otimes f^{i} \\
& =\Upsilon_{a_{(2)}} \otimes S\left(a_{(1)}\right) a_{(3)} .
\end{aligned}
$$

(Note the appearance of the adjoint coaction (3.23) in this equation.) Therefore, we find that $\mathcal{A}$ right coacts on $Y$ as

$$
\Delta_{\mathcal{A}}\left(Y^{i}{ }_{j}\right)=Y_{\ell}^{k} \otimes S\left(A^{i}{ }_{k}\right) A^{\ell}{ }_{j}
$$

The above calculation has an added bonus: (4.26) tells us that the adjoint action of $\mathcal{U}$ on any element in the subspace $\left\{\Upsilon_{a} \mid a \in \mathcal{A}\right\}$ returns another element of the same subspace. In particular,

$$
\underset{x \triangleright Y_{j}^{i}}{\operatorname{ad}}=\left\langle x, S\left(A_{k}^{i}\right) A_{j}^{\ell}\right\rangle Y_{\ell}^{k}
$$

which is simply a linear combination of the entries of $Y$.

Notice that in the classical limit, since $\mathcal{R} \rightarrow 1_{\mathcal{U}} \otimes 1_{\mathcal{U}}, Y \rightarrow I 1_{\mathcal{U}}$; therefore, we can define the matrix $X$ by

$$
X:=\frac{I 1_{u}-Y}{\lambda}
$$

where, as always, $\lambda=q-\frac{1}{q}$. Thus, in the $q \rightarrow 1$ limit, $X$ is well-defined. However, the real reason for defining this new matrix becomes apparent when we look at its properties which follow from those of $Y$ : the relevant commutation relations are

$$
\begin{gathered}
L_{1}^{+} X_{2}=R_{21}^{-1} X_{2} R_{21} L_{1}^{+}, L_{1}^{-} X_{2}=R X_{2} R^{-1} L_{1}^{-}, \\
R_{21} X_{1} R X_{2}-X_{2} R_{21} X_{1} R=\frac{1}{\lambda}\left(R_{21} R X_{2}-X_{2} R_{21} R\right),
\end{gathered}
$$

and the Hopf algebra properties of $X$ are

$$
\begin{gathered}
\Delta\left(X^{i}{ }_{j}\right)=X^{i}{ }_{j} \otimes 1_{u}+\left(L^{+}\right)_{k}^{i} S\left(\left(L^{-}\right)^{\ell}{ }_{j}\right) \otimes X^{k}{ }_{\ell,} \quad \epsilon(X)=0, \\
S\left(X^{i}{ }_{j}\right)=-S^{2}\left(\left(L^{-}\right)^{\ell}{ }_{j}\right) S\left(\left(L^{+}\right)^{i}{ }_{k}\right) X^{k}{ }_{\ell} .
\end{gathered}
$$

It follows immediately that $\mathcal{A}$ left and right coacts on $X$ exactly as it does on $Y$, i.e.

$$
{ }_{\mathcal{A}} \Delta\left(X^{i}{ }_{j}\right)=1_{\mathcal{A}} \otimes X^{i}{ }_{j}, \quad \Delta_{\mathcal{A}}\left(X^{i}{ }_{j}\right)=X^{k}{ }_{\ell} \otimes S\left(A^{i}{ }_{k}\right) A^{\ell}{ }_{j}
$$


The adjoint action of $x \in \mathcal{U}$ on an entry of $X$ is given by (4.29) with $Y$ replaced by $X$, and this returns an element in $\mathrm{g}$, the subspace of $\mathcal{U}$ defined to be the span of the entries of $X$ over $k$. Furthermore, $\epsilon(\mathrm{g})=0$; thus, the UEA $U_{q}(\mathrm{~g})$ satisfies all criteria needed for a QLA. We therefore see that any quasitriangular Hopf algebra, together with a representation, allows the construction of a QLA. The connection to the contents of Section 4.1 is made by taking the capital roman indices to correspond to pairs of small roman indices in the present quasitriangular case. To see how this is done, we compute the adjoint action of an element of $X$ on another:

$$
\begin{aligned}
X^{i}{ }_{j \triangleright X_{\ell} X_{\ell}} & =X^{i}{ }_{j} X^{k}{ }_{\ell}-\left(R_{21}^{-1} X_{2} R_{21} X_{1} R\right)^{i m}{ }_{n \ell} \tilde{R}^{n k}{ }_{j m} \\
& =\tilde{R}^{s k}{ }_{j r} R^{r i}{ }_{n m}\left(R_{21} X_{1} R X_{2}-X_{2} R_{21} X_{1} R\right)^{m n}{ }_{s \ell} \\
& =\frac{1}{\lambda}\left[\delta_{j}^{i} X^{k}{ }_{\ell}-\left(R_{21}^{-1} X_{2} R_{21} R\right)^{i m}{ }_{n \ell} \tilde{R}^{n k}{ }_{j m}\right] .
\end{aligned}
$$

Comparison with (4.10) motivates the definitions of the generators, $\mathcal{O}_{s}$, and adjoint matrices as

$$
\chi_{(i j)}:=X^{i}{ }_{j}, \quad \mathcal{O}_{(i j)}{ }^{(k \ell)}:=\left(L^{+}\right)^{i}{ }_{k} S\left(\left(L^{-}\right)^{\ell}{ }_{j}\right), \quad \mathrm{A}^{(i j)}{ }_{(k \ell)}:=S\left(A^{k}{ }_{i}\right) A^{j}{ }_{\ell},
$$

and the R-matrix and structure constants as

$$
\begin{aligned}
\hat{\mathrm{R}}^{(a b)(c d)}{ }_{(i j)(k \ell)} & :=\tilde{R}^{m k}{ }_{j n} \hat{R}^{s d}{ }_{m \ell}\left(\hat{R}^{-1}\right)^{n i}{ }_{r a} \hat{R}^{r b}{ }_{s c}, \\
f_{(i j)(k \ell)}{ }^{(r s)} & :=\frac{1}{\lambda}\left[\delta_{j}^{i} \delta_{r}^{k} \delta_{\ell}^{s}-\tilde{R}^{m k}{ }_{j n}\left(\hat{R}^{-1}\right)^{n i}{ }_{t r}\left(\hat{R}^{2}\right)^{t s}{ }_{m \ell}\right] .
\end{aligned}
$$

However, notice that the universal R-matrix in this representation, i.e. $\mathrm{R}^{A B} C D:=$ $\left\langle\mathcal{R}, \mathrm{A}^{A}{ }_{C} \otimes \mathrm{A}^{B}{ }_{D}\right\rangle$, is

$$
\begin{aligned}
\mathbf{R}_{(i j)(k \ell)}^{(a b)(c d)} & =\left\langle\mathcal{R}, S\left(A_{a}^{i}\right) A^{b}{ }_{j} \otimes S\left(A^{k}{ }_{c}\right) A^{d}{ }_{\ell}\right\rangle \\
& =\tilde{R}^{m k}{ }_{j n} \hat{R}^{s b}{ }_{m \ell} \hat{R}^{n i}{ }_{r c}\left(\hat{R}^{-1}\right)^{r d}{ }_{s a}
\end{aligned}
$$

which is not equal to $\hat{\mathbf{R}}^{(c d)(a b)}{ }_{(i j)(k \ell)}$.

\subsubsection{Example: $U_{q}(s l(2))$}

To provide a concrete example of the results of the previous subsection, we consider the QLA $U_{q}(s l(2))$ : define $\chi_{1}, \chi_{+}, \chi_{-}$and $\chi_{2}$ to be the entries of the $2 \times 2$ matrix of generators $X$, i.e.

$$
X=\left(\begin{array}{cc}
\chi_{1} & \chi_{+} \\
\chi_{-} & \chi_{2}
\end{array}\right)
$$


Now, putting the R-matrix for $S L_{q}(2)$, i.e. (2.32), into the expression for $\hat{\mathrm{R}}$ from above, we find the $16 \times 16$ matrix

$$
\left(\begin{array}{cccccccccccccccc}
1 & 0 & 0 & 0 & 0 & 0 & 0 & 0 & 0 & 0 & 0 & 0 & 0 & 0 & 0 & 0 \\
0 & \frac{\lambda}{q} & 0 & 0 & 1 & 0 & 0 & 0 & 0 & 0 & 0 & 0 & 0 & 0 & 0 & 0 \\
0 & 0 & -q \lambda & 0 & 0 & 0 & 0 & 0 & 1 & 0 & 0 & 0 & 0 & 0 & 0 & 0 \\
\frac{\lambda^{2}}{q^{2}} & 0 & 0 & -\lambda^{2} & 0 & 0 & -\frac{\lambda}{q} & 0 & 0 & \frac{\lambda}{q} & 0 & 0 & 1 & 0 & 0 & 0 \\
0 & \frac{1}{q^{2}} & 0 & 0 & 0 & 0 & 0 & 0 & 0 & 0 & 0 & 0 & 0 & 0 & 0 & 0 \\
0 & 0 & 0 & 0 & 0 & 1 & 0 & 0 & 0 & 0 & 0 & 0 & 0 & 0 & 0 & 0 \\
\frac{\lambda}{q} & 0 & 0 & -q \lambda & 0 & 0 & 0 & 0 & 0 & 1 & 0 & 0 & 0 & 0 & 0 & 0 \\
0 & \frac{\left(q^{4}-1\right) \lambda}{q^{3}} & 0 & 0 & \frac{\lambda}{q} & 0 & 0 & -q \lambda & 0 & 0 & 0 & 0 & 0 & q^{2} & 0 & 0 \\
0 & 0 & q^{2} & 0 & 0 & 0 & 0 & 0 & 0 & 0 & 0 & 0 & 0 & 0 & 0 & 0 \\
-\frac{\lambda}{q} & 0 & 0 & q \lambda & 0 & 0 & 1 & 0 & 0 & 0 & 0 & 0 & 0 & 0 & 0 & 0 \\
0 & 0 & 0 & 0 & 0 & 0 & 0 & 0 & 0 & 0 & 1 & 0 & 0 & 0 & 0 & 0 \\
0 & 0 & 0 & 0 & 0 & 0 & 0 & 0 & -\frac{\lambda}{q^{3}} & 0 & 0 & \frac{\lambda}{q} & 0 & 0 & \frac{1}{q^{2}} & 0 \\
0 & 0 & 0 & 1 & 0 & 0 & 0 & 0 & 0 & 0 & 0 & 0 & 0 & 0 & 0 & 0 \\
0 & -\frac{\lambda}{q} & 0 & 0 & 0 & 0 & 0 & 1 & 0 & 0 & 0 & 0 & 0 & 0 & 0 & 0 \\
0 & 0 & q \lambda & 0 & 0 & 0 & 0 & 0 & 0 & 0 & 0 & 1 & 0 & 0 & 0 & 0 \\
-\frac{\lambda^{2}}{q^{2}} & 0 & 0 & \lambda^{2} & 0 & 0 & \frac{\lambda}{q} & 0 & 0 & -\frac{\lambda}{q} & 0 & 0 & 0 & 0 & 0 & 1
\end{array}\right)
$$

where we have taken the ordered basis $\left\{\chi_{1}, \chi_{+}, \chi_{-}, \chi_{2}\right\}$. The nonvanishing structure constants, also using the expression given, are

$$
\begin{array}{llll}
f_{11}{ }^{1}=-\frac{\lambda}{q^{2}}, & f_{11}{ }^{2}=\frac{\lambda}{q^{2}}, & f_{1+}{ }^{+}=\frac{1}{q}+\frac{1}{q^{3}}-q, & f_{1-}{ }^{-}=-q, \\
f_{12}{ }^{2}=\lambda, & f_{12}{ }^{2}=-\lambda, & f_{2+}{ }^{+}=-q, & f_{2-}=\frac{1}{q}, \\
f_{+1}{ }^{+}=-\frac{1}{q}, & f_{+-}{ }^{1}=\frac{1}{q}, & f_{+-}{ }^{2}=-\frac{1}{q}, & f_{+2}{ }^{+}=q, \\
f_{-1}{ }^{-}=\frac{1}{q^{3}}, & f_{-+}{ }^{1}=-\frac{1}{q}, & f_{-+}{ }^{2}=\frac{1}{q}, . & f_{-2^{-}}=-\frac{1}{q} .
\end{array}
$$

Naturally, in the $q \rightarrow 1$ limit, we get $\hat{\mathrm{R}}^{A B} C D=\delta_{D}^{A} \delta_{C}^{B}$ and the correct (antisymmetric) structure constants for $s l(2)$.

So what commutation relations do these give? They take the form

$$
\begin{array}{r}
\chi_{1} \chi_{+}=\chi_{+} \chi_{1}+\frac{1}{q} \chi_{+}-\frac{\lambda}{q} \chi_{+} \chi_{2}, \quad \chi_{1} \chi_{-}=\chi_{-} \chi_{1}-\frac{1}{q} \chi_{-}+\frac{\lambda}{q} \chi_{2} \chi_{-}, \\
\chi_{2} \chi_{+}=q^{2} \chi_{+} \chi_{2}-q \chi_{+}, \quad \chi_{2} \chi_{-}=\frac{1}{q^{2}} \chi_{-} \chi_{2}+\frac{1}{q} \chi_{-}, \\
\chi_{+} \chi_{-}=\chi_{-} \chi_{+}+\frac{1}{q}\left(1_{\mathcal{U}}-\lambda \chi_{2}\right)\left(\chi_{1}-\chi_{2}\right), \\
\chi_{1} \chi_{2}=\chi_{2} \chi_{1} .
\end{array}
$$


However, for the purposes of the next subsection, it becomes convenient to change bases by defining the generators $\chi_{0}:=\chi_{1}-\chi_{2}$ and $\mathcal{X}:=\chi_{1}+\frac{1}{q^{2}} \chi_{2}$. The usefulness of these is apparent when we consider the adjoint actions in this new basis: we find

$$
\mathcal{X}_{\triangleright}^{\mathrm{ad}} \mathcal{X}=0, \quad \chi_{i}^{\mathrm{ad}} \stackrel{\mathcal{X}}{\mathrm{a}}=0, \quad \mathcal{X}_{\triangleright}^{\mathrm{ad}} \chi_{i}=-\frac{1}{q}\left(q^{2}-\frac{1}{q^{2}}\right) \chi_{i}
$$

(where $i=0,+,-$ ), as well as

$$
\chi_{0} \triangleright \chi_{0}^{\mathrm{ad}}=-\frac{1}{q}\left(q^{2}-\frac{1}{q^{2}}\right) \chi_{0}, \quad \chi_{0}^{\mathrm{ad}} \chi_{ \pm}= \pm q^{\mp 1}\left(1+\frac{1}{q^{2}}\right) \chi_{ \pm} .
$$

It is also interesting to note that by using (4.41), we discover that $\mathcal{X}$ is central in the algebra; we will have more to say on this a bit later.

\subsection{The Killing Metric}

\subsubsection{The Killing Form for a Quasitriangular Hopf Alge- bra}

Let $\mathcal{U}$ be a quasitriangular Hopf algebra with R-matrix $\mathcal{R}$, and $\rho: \mathcal{U} \rightarrow M_{N}(k)$ be an $N \times N$ matrix representation of $\mathcal{U}$ with entries in $k$. We may define the bilinear map $\eta^{(\rho)}: \mathcal{U} \otimes \mathcal{U} \rightarrow k$, the Killing form associated with the representation $\rho$, as

$$
\eta^{(\rho)}(x \otimes y):=\operatorname{tr}_{\rho}(u x y)
$$

where $x, y \in \mathcal{U}, \operatorname{tr}_{\rho}$ is the trace over the given representation, and $u$ is the generator of the square of the antipode (see Appendix A). $\eta^{(\rho)}$ has the following properties:

$$
\begin{aligned}
\eta^{(\rho)}(y \otimes x) & =\eta^{(\rho)}\left(x \otimes S^{2}(y)\right), \\
\eta^{(\rho)}\left(\left(z_{(1)} \stackrel{\text { ad }}{\triangleright} x\right) \otimes\left(z_{(2)} \stackrel{\text { dd }}{\triangleright}\right)\right) & =\eta^{(\rho)}(x \otimes y) \epsilon(z),
\end{aligned}
$$

for all $x, y, z \in \mathcal{U}$. The first of these identities expresses the "symmetry" of $\eta^{(\rho)}$, and immediately follows from the properties of $u$; the second is a statement of the invariance of the Killing form under the adjoint action of $\mathcal{U}$ on itself, and comes from the fact that

$$
\operatorname{tr}_{\rho}(u(x \triangleright d))=\operatorname{tr}_{\rho}\left(u x_{(1)} y S\left(x_{(2)}\right)\right)
$$




$$
\begin{aligned}
& =\eta^{(\rho)}\left(x_{(1)} \otimes y S\left(x_{(2)}\right)\right) \\
& =\eta^{(\rho)}\left(y S\left(x_{(2)}\right) \otimes S^{2}\left(x_{(1)}\right)\right) \\
& =\operatorname{tr}_{\rho}\left(u y S\left(S\left(x_{(1)}\right) x_{(2)}\right)\right) \\
& =\operatorname{tr}_{\rho}(u y) \epsilon(x) .
\end{aligned}
$$

The invariance under the adjoint action may be thought of as how the Killing form behaves under an "infinitesimal" transformation; as remarked in Chapter 3, the "finite" transformation is given by the right coaction (3.34) of the dually paired Hopf algebra $\mathcal{A}$ on $\mathcal{U}$, and the Killing form has the property

$$
\begin{aligned}
\eta^{(\rho)}\left(x^{(1)} \otimes y^{(1)}\right) x^{(2)^{\prime}} y^{(2)^{\prime}} & =\eta^{(\rho)}\left(\left(e_{i}^{\mathrm{ad} \triangleright x}\right) \otimes\left(e_{j}^{\mathrm{ad}} y\right)\right) f^{i} f^{j} \\
& =\eta^{(\rho)}\left(\left(\left(e_{i}\right)_{(1)} \stackrel{\mathrm{ad}}{\triangleright}\right) \otimes\left(\left(e_{i}\right)_{(2)} \underset{\mathrm{ad}}{\triangleright} y\right)\right) f^{i} \\
& =\eta^{(\rho)}(x \otimes y) \epsilon\left(e_{i}\right) f^{i} \\
& =\eta^{(\rho)}(x \otimes y) 1_{\mathcal{A}} .
\end{aligned}
$$

This is therefore the "finite" version of the invariance of $\eta^{(\rho)}$.

\subsubsection{The Killing Metric for a Quantum Lie Algebra}

In the case when $\mathcal{U}$ is not only quasitriangular, but also a QLA with generators $\left\{\chi_{A}\right\}$, we can define the Killing metric associated with the representation $\rho$ as

$$
\eta_{A B}^{(\rho)}:=\eta^{(\rho)}\left(\chi_{A} \otimes \chi_{B}\right)
$$

It is now convenient to define the quantity $\mathcal{I}_{A}^{(\rho)}:=-\operatorname{tr}_{\rho}\left(u \chi_{A}\right)$ (the sign is merely a convention); from the results of the previous subsection,

$$
\begin{aligned}
\operatorname{tr}_{\rho}\left(u\left(\chi_{A} \stackrel{\mathrm{ad}}{\triangleright} \chi_{B}\right)\right) & =-f_{A B}{ }^{C} \mathcal{I}_{C}^{(\rho)}=0, \\
\operatorname{tr}_{\rho}\left(u\left(\mathcal{O}_{A}{ }^{B_{\triangleright}^{\mathrm{ad}}} \chi_{C}\right)\right) & =-\hat{\mathrm{R}}^{D B} \cdot{ }_{A C} \mathcal{I}_{D}^{(\rho)}=-\delta_{B}^{A} \mathcal{I}_{C}^{(\rho)}, \\
\operatorname{tr}_{\rho}\left(u \chi_{B}\right) A^{B}{ }_{A} & =-\mathcal{I}_{B}^{(\rho)} A^{B}{ }_{A}=-\mathcal{I}_{A}^{(\rho)} 1_{A} .
\end{aligned}
$$

The first of (4.49) implies that if we multiply (4.10) by $u$ and trace over a representation $\rho$, we find that the Killing metric satisfies

$$
\eta_{A B}^{(\rho)}=\hat{\mathbf{R}}_{A B}^{C D} \eta_{C D}^{(\rho)}
$$


which gives the "symmetry" of the Killing metric". We can also obtain the "total antisymmetry" of the structure constants in a similar way; since the counit of all the generators vanish, (4.45) requires that

$$
\begin{aligned}
& 0=\eta^{(\rho)}\left(\left(\chi_{C(1)} \stackrel{a d}{\triangleright} \chi_{A}\right) \otimes\left(\chi_{C(2)} \stackrel{a d}{\triangleright d} \chi_{B}\right)\right) \\
& =\eta^{(\rho)}\left(\left(\chi_{C} \stackrel{\text { ad }}{\triangleright \chi_{A}}\right) \otimes \chi_{B}\right)+\eta^{(\rho)}\left(\left(\mathcal{O}_{C}{ }^{D \stackrel{a d}{\triangleright} \chi_{A}}\right) \otimes\left(\chi_{D} \stackrel{\text { ad }}{\triangleright \chi_{B}}\right)\right) \\
& =f_{C A}{ }^{D} \eta^{(\rho)}\left(\chi_{D} \otimes \chi_{B}\right)+\hat{\mathbf{R}}^{E D}{ }_{C A} f_{D B}{ }^{F} \eta^{(\rho)}\left(\chi_{E} \otimes \chi_{F}\right) \text {, }
\end{aligned}
$$

so we find that

$$
f_{C A}{ }^{D} \eta_{D B}^{(\rho)}+\hat{\mathbf{R}}^{E D}{ }_{C A} f_{D B}{ }^{F} \eta_{E F}^{(\rho)}=0 .
$$

If we use (4.16), together with (4.47), we see that the invariance of the Killing metric under finite rotations takes the form

$$
\eta_{C D}^{(\rho)} A^{C}{ }_{A} A_{B}^{D}=\eta_{A B}^{(\rho)} 1_{A}
$$

\section{Quadratic Casimirs}

Now, suppose that $\eta_{A B}^{(\rho)}$ is invertible, i.e. there exists a numerical matrix $\eta^{(\rho) A B}$ such that

$$
\eta_{A C}^{(\rho)} \eta^{(\rho) C B}=\eta^{(\rho) B C} \eta_{C A}^{(\rho)}=\delta_{A}^{B} .
$$

Then (4.53) implies that $A^{A}{ }_{C} A^{B}{ }_{D} \eta^{(\rho) C D}=\eta^{(\rho) A B} 1_{\mathcal{A}}$; this in turn indicates that the quantum quadratic Casimir defined by

$$
Q_{2}^{(\rho)}:=\eta^{(\rho) A B} \chi_{A} \chi_{B}
$$

is central. Why? Firstly, note that $Q_{2}^{(\rho)}$ is right-invariant:

$$
\begin{aligned}
\Delta_{\mathcal{A}}\left(Q_{2}^{(\rho)}\right) & =\eta^{(\rho) A B} \Delta_{\mathcal{A}}\left(\chi_{A}\right) \Delta_{\mathcal{A}}\left(\chi_{B}\right) \\
& =\chi_{C} \chi_{D} \otimes \eta^{(\rho) A B} \mathrm{~A}^{C}{ }_{A} \mathrm{~A}^{D}{ }_{B} \\
& =\chi_{C} \chi_{D} \eta^{(\rho) C D} \otimes 1_{\mathcal{A}} \\
& =Q_{2}^{(\rho)} \otimes 1_{\mathcal{A}} .
\end{aligned}
$$

Now, recall (3.31) and (4.8); the first of these states that if $x$ is right-invariant,

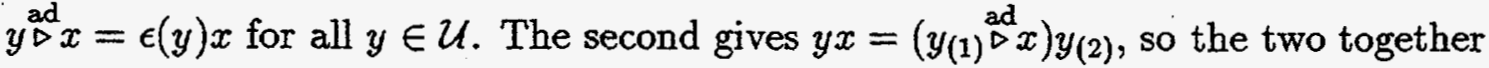

\footnotetext{
${ }^{1}$ This equation also explicitly shows the existence of an eigenvector of $\hat{\mathbf{R}}$ with eigenvalue 1 , so the frequently occuring combination $\hat{R}-I$ is noninvertible.
} 
imply that $x y=y x$, namely, any right-invariant element of $\mathcal{U}$ is central. Since we have just shown right-invariance of $Q_{2}^{(\rho)}$, it follows that the quantum quadratic Casimir commutes with everything in the algebra, just as in the classical case.

\subsubsection{Examples}

We now present some explicit examples of some of the results in the previous subsections. These will hopefully illustrate many of the concepts we have just encountered.

Fundamental Representations of $G L_{q}(2)$ and $S L_{q}(2)$

As our first example, we compute what the Killing metrics for the fundamental representations of $G L_{q}(2)$ and $S L_{q}(N)$. These may be considered together due to the fact that their R-matrices differ only by a factor of $q^{-\frac{1}{2}}$. In the basis $\left(\chi_{1}, \chi_{+}, \chi_{-}, \chi_{2}\right)$ ) given by (4.38), we find

$$
\left(\chi_{i j}\right)_{\ell}^{k}=\left(\frac{I-\hat{R}^{2}}{\lambda}\right)_{j \ell}^{i k}
$$

where $\hat{R}$ is given through (2.32) for $S L_{q}(2)$, and $r^{-1}=q^{\frac{1}{2}}$ times this for $G L_{q}(2)$. However, it is somewhat more useful to use $\mathcal{X}$ and $\chi_{0}$ rather than $\chi_{1}$ and $\chi_{2}$; when we do this, we find that

$$
\begin{aligned}
& \mathcal{X}=\frac{1}{\lambda}\left(1+\frac{1}{q^{2}}-r^{2}\left(q^{2}+\frac{1}{q^{2}}\right)\right)\left(\begin{array}{cc}
1 & 0 \\
0 & 1
\end{array}\right), \chi_{+}=-r^{2}\left(\begin{array}{ll}
0 & 0 \\
1 & 0
\end{array}\right), \\
& \chi_{-}=-r^{2}\left(\begin{array}{ll}
0 & 1 \\
0 & 0
\end{array}\right), \chi_{0}=r^{2}\left(\begin{array}{cc}
-q & 0 \\
0 & \frac{1}{q}
\end{array}\right),
\end{aligned}
$$

and also

$$
u=\frac{1}{r q^{3}}\left(\begin{array}{cc}
1 & 0 \\
0 & q^{2}
\end{array}\right) .
$$

Thus, when we compute $\eta^{(\rho)}$ in the basis $\left(\mathcal{X}, \chi_{+}, \chi_{-}, \chi_{0}\right)$ with the appropriate value for $r$ stuck in, we find

$$
\eta^{\left(\text {fund } G L_{q}(2)\right)}=\frac{1}{q^{2}}\left(\begin{array}{cccc}
q^{2}\left(q+\frac{1}{q}\right) & 0 & 0 & 0 \\
0 & 0 & q & 0 \\
0 & \frac{1}{q} & 0 & 0 \\
0 & 0 & 0 & q+\frac{1}{q}
\end{array}\right)
$$




$$
\eta^{\text {(fund } \left.S L_{q}(2)\right)}=q^{-\frac{7}{2}}\left(\begin{array}{cccc}
\frac{1}{q^{2}\left(\frac{q^{3}-1}{q+1}\right)^{2}\left(q+\frac{1}{q}\right)} & 0 & 0 & 0 \\
0 & 0 & q & 0 \\
0 & \frac{1}{q} & 0 & 0 \\
0 & 0 & 0 & q+\frac{1}{q}
\end{array}\right)
$$

Except for an overall factor, we see that the lower right-hand $3 \times 3$ matrices are the same, whereas the upper left-hand entry vanishes in the classical limit for $S L_{q}(2)$. This is not surprising, since it corresponds to the fact that classical $S L(2)$ has only three generators, not four.

For $q \neq 1$, both of these Killing metrics are invertible, and thus the quadratic Casimirs can be found. When we do the calculations for these representations, we find

$$
Q_{2}^{\left(\mathrm{fund} G / S L_{q}(2)\right)}=r q[2]_{q}\left(\begin{array}{ll}
1 & 0 \\
0 & 1
\end{array}\right),
$$

so, as we'd expect, it is proportional to the $2 \times 2$ identity matrix.

\section{Adjoint Representation of $S L_{q}(2)$}

Using the structure constants for $U_{q}(s l(2))$ from before, we find the generators in the adjoint representation:

$$
\begin{gathered}
\mathcal{X}=-\frac{\lambda}{q}\left(\begin{array}{cccc}
\frac{1}{q} & -q & 0 & 0 \\
-\frac{1}{q} & q & 0 & 0 \\
0 & 0 & q+\frac{1}{q} & 0 \\
0 & 0 & 0 & q+\frac{1}{q}
\end{array}\right), \chi_{+}=\left(\begin{array}{cccc}
0 & 0 & 0 & \frac{1}{q} \\
0 & 0 & 0 & -\frac{1}{q} \\
-\frac{1}{q} & q & 0 & 0 \\
0 & 0 & 0 & 0
\end{array}\right), \\
\chi-=\frac{1}{q^{2}}\left(\begin{array}{cccc}
0 & 0 & -q & 0 \\
0 & 0 & q & 0 \\
0 & 0 & 0 & 0 \\
\frac{1}{q} & -q & 0 & 0
\end{array}\right), \chi_{0}=\frac{1}{q}\left(\begin{array}{cccc}
-\frac{\lambda}{q} & q \lambda & 0 & 0 \\
\frac{\lambda}{q} & -q \lambda & 0 & 0 \\
0 & 0 & 1+\frac{1}{q^{2}} & 0 \\
0 & 0 & 0 & -\left(1+q^{2}\right)
\end{array}\right),
\end{gathered}
$$

and $u$ is

$$
u=\left(\begin{array}{cccc}
1-\frac{1}{q^{2}}+\frac{1}{q^{4}} & \frac{\lambda}{q} & 0 & 0 \\
\frac{\lambda}{q^{3}} & \frac{1}{q^{2}} & 0 & 0 \\
0 & 0 & \frac{1}{q^{2}} & 0 \\
0 & 0 & 0 & \frac{1}{q^{6}}
\end{array}\right)
$$


The Killing metric, which is just $f_{A C}{ }^{D} f_{B D}{ }^{C}$ in this representation, is therefore

$$
\begin{aligned}
& \eta_{A B}^{\left(\text {adj } S L_{q}(2)\right)}= \\
& \frac{q+\frac{1}{q}}{q^{6}}\left(\begin{array}{cccc}
\frac{\lambda^{2}}{q^{2}}\left(q+\frac{1}{q}\right)[3]_{q} & 0 & 0 & 0 \\
0 & 0 & q\left(q^{2}+\frac{1}{q^{2}}\right) & 0 \\
0 & \frac{1}{q}\left(q^{2}+\frac{1}{q^{2}}\right) & 0 & 0 \\
0 & 0 & 0 & \left(q^{2}+\frac{1}{q^{2}}\right)\left(q+\frac{1}{q}\right)
\end{array}\right)
\end{aligned}
$$

Once again, for $q \neq 1$, this is invertible, and the quadratic Casimir comes out to be

$$
Q_{2}^{\left(\operatorname{adj} S L_{q}(2)\right)}=\frac{q^{4}[2]_{q}}{[3]_{q}}\left(\begin{array}{cccc}
1 & -q^{2} & 0 & 0 \\
-1 & q^{2} & 0 & 0 \\
0 & 0 & {[2]_{q}} & 0 \\
0 & 0 & 0 & {[2]_{q}}
\end{array}\right) .
$$

This matrix has a zero eigenvalue and three degenerate eigenvalues of $q^{4}[2]_{q}^{2} /[3]_{q}$, so it can be block-diagonalized into a $(1 \times 1) \oplus(3 \times 3)$ matrix. (This is the first indication that the adjoint representation for $S L_{q}(2)$ is reducible, and we will come back to this point shortly.)

Up to multiplicative factors, the lower right-hand $3 \times 3$ submatrices of $(4.60)$ and (4.64) are the same. However, recall that there is a general theorem for compact Lie algebras in the classical case: for a given basis of generators, all Killing metrics computed from irreducible representations are proportional. The appearance of the same matrix in the three cases considered above is an indication that perhaps there is an analagous theorem for the deformed case as well. In fact, consider the classical case of $S U(N)$; up to an overall normalization, the quadratic Casimir in the fundamental representation is proportional to $N^{2}-1$, and for the adjoint representation, it is the same constant times $N$, so the ratio between the former and the latter is $\frac{N^{2}-1}{N}$. For the $S L_{q}(2)$ cases we have just studied, this ratio is

$$
Q_{2}^{\left(\text {fund } S L_{q}(2)\right)} / Q_{2}^{\left(\text {adj } S L_{q}(2)\right)}=q^{-\frac{7}{2}} \frac{[3]_{q}}{[2]_{q}}
$$

which agrees exactly with the classical case in the $q \rightarrow 1$ limit for $N=2$.

We also note that if we instead choose the basis $\left(\mathcal{X}, \chi_{-},\left(q+\frac{1}{q}\right)^{-\frac{1}{2}} \chi_{0}, \chi_{-}\right)$, this $3 \times 3$ matrix would be proportional to

$$
\left(\begin{array}{ccc}
0 & 0 & \frac{1}{q} \\
0 & 1 & 0 \\
q & 0 & 0
\end{array}\right)
$$


which is the metric for $S \mathrm{q}_{q^{2}}(3)$. There is prior evidence for the equivalence of this quantum group with $S L_{q}(2)$ (just as in the classical case) [23], and our result here supports this.

\subsection{Some Comments on the Adjoint Represen- tation}

To conclude this chapter, we examine some of the implications of (4.49). Notice that unless $\mathcal{I}_{A}^{(\rho)}$ vanishes identically for all representations, we are able to deduce the existence of another numerical object $\mathcal{D}^{A}$ which satisfies

$$
f_{A B}^{C} \mathcal{D}^{B}=0
$$

Why should this quantity exist? From the last of $(4.49), \mathcal{I}_{A}^{(\rho)} 1_{\mathcal{A}}$ is an algebra-valued eigenvector of $A^{t}$ with eigenvalue unity. The transpose of any matrix has the same eigenvalues as the original, so this implies the existence of a numerical quantity $\mathcal{D}^{A}$ such that $\mathcal{D}^{A} 1_{\mathcal{A}}$ is the algebra-valued eigenvector of $A$ with unit eigenvalue, i.e.

$$
A^{A}{ }_{B} \mathcal{D}^{B}=\mathcal{D}^{A} 1_{\mathcal{A}}
$$

This in turn implies that

$$
f_{A B}^{C} \mathcal{D}^{B}=0, \quad \hat{\mathbf{R}}^{C A}{ }_{B D} \mathcal{D}^{D}=\delta_{B}^{A} \mathcal{D}^{C} .
$$

The first of these equations implies that $\mathcal{D}^{A}$ is a nonzero null eigenvector for all the generator matrices in the adjoint representation, so if there does indeed exist a representation for which $\operatorname{tr}_{\rho}\left(u \chi_{A}\right)$ does not vanish, the adjoint representation is reducible. (In fact, when we computed the quadratic Casimir for $S L_{q}(2)$ in this representation, there were already hints of this result.) Since we know that the adjoint is irreducible for the classical compact Lie algebras, this indicates that as $q \rightarrow 1, \operatorname{tr}_{\rho}\left(u \chi_{A}\right) \rightarrow 0$ for all representations, so tracelessness of the generators is recovered.

Another consequence is that the quantity $\mathcal{D}^{A} \chi_{A}$ is central, for precisely the same reason that $Q_{2}^{(\rho)}$ is, namely, it is right-invariant. This follows immediately from the definition of $\mathcal{D}^{A}$ given above in (4.69).

The normalizations of $\mathcal{I}_{A}^{(\rho)}$ and $\mathcal{D}^{A}$ are not fixed by their definitions; they are both arbitrary up to multiplicative factors. However, if we wanted to, we could 
eliminate one of these factors in terms of the other by fixing the product $\mathcal{I}_{A}^{(\rho)} \mathcal{D}^{A}$ to be some convenient number.

For an explicit example, we look at the case in which the QLA in question is one constructed from a quasitriangular Hopf algebra. Consider the third of equations (4.49); the explicit form of the adjoint matrices $A$ in (4.35) implies that if a nonvanishing $\mathcal{I}_{A}^{(\rho)}$ exists, the matrix $\mathcal{I}^{i}{ }_{j}:=\mathcal{I}_{(i j)}^{\rho}$ must satisfy $A \mathcal{I}=\mathcal{I} A$. The only matrices which satisfy this relation are multiples of the identity; it is easily shown that such matrices also satisfy the first two of (4.49) as well. Therefore, for such QLAs, we choose the canonical form $\mathcal{I}_{(i j)}^{(\rho)}:=\kappa \delta_{j}^{i}$, and compute $\kappa$ accordingly. We also find that $\mathcal{D}_{(i j)}$ must be proportional to $\left(D^{-1}\right)_{i}^{j}$, so that $\mathcal{I}_{A}^{(\rho)} \mathcal{D}^{A} \propto \operatorname{tr}\left(D^{-1}\right)$. This expression for $\mathcal{D}^{A}$ also indicates that $\mathcal{D}^{A} \chi_{A}=\operatorname{tr}\left(D^{-1} X\right)$, which we know from Appendix A.1 is right-invariant, and therefore commutes with every element of the QLA. In fact, for the $S L_{q}(2)$ case, this is just proportional to $\mathcal{X}$ from the previous section, which we saw from the explicit commutation relations was indeed central.

The fundamental representations of the quantum Lie groups in Appendix A satisfy the above criteria, i.e. the quantities $\mathcal{I}_{(i j)}^{(\text {fund) }}$ are all nonzero, provided $q \neq 1$. The values of $\kappa$ therefore can be computed, and are:

$$
\begin{aligned}
\kappa\left(G L_{q}(N)\right) & =1 \\
\kappa\left(S L_{q}(N)\right) & =q^{-\frac{1}{N}}\left(1-\left[\frac{1}{N}\right]_{q}[N]_{\frac{1}{q}}\right) \\
\kappa\left(S O_{q}(N) / S P_{q}\left(\frac{1}{2} N\right)\right) & =q^{N-\epsilon}-q^{\epsilon-N}
\end{aligned}
$$

(where we have combined the orthogonal and symplectic groups by using the quantity $\epsilon= \pm 1$ ). Looking at the values of $\kappa$ given above, we see that they vanish in the classical limit for $S L_{q}(N), S O_{q}(N)$, and $S P_{q}\left(\frac{1}{2} N\right)$. This must happen, since we know that in the classical case, the adjoint representation is irreducible. $\kappa\left(G L_{q}(N)\right)$ is nonzero for all values of $q$, but this is not surprising, since $G L(N)$ is not compact, and its adjoint representation is indeed reducible. 


\section{Chapter 5}

\section{Cartan Calculus on Hopf Algebras and Quantum Lie Algebras}

The purpose of this chapter is to generalize the classical case, and it builds upon the structure of the universal differential calculus associated with a Hopf algebra. (For readers unfamiliar with the classical Cartan calculus, Appendix B contains the basic background material and references.) The basics of this approach are discussed in Appendix C, and the reader unfamiliar with the subject should look therein before proceeding, if only to familiarize him- or herself with the notation we use here. Our method of attack will be to start with the UDC $(\Omega(\mathcal{A}), \delta)$ of a Hopf algebra $\mathcal{A}$, and introduce Lie derivatives and inner derivations which act on $\Omega(\mathcal{A})$. Our "deformed" version presented here will allow for possible noncommutativity of the elements of $\Omega(\mathcal{A})$, unlike the classical case. However, as in the latter, we need specify only how the derivations act on and commute with 0 - and I-forms; the extension to arbitrary $p$-forms in $\Omega(\mathcal{A})$ follows immediately.

\subsection{Universal Cartan Calculus}

We begin with two dually paired Hopf algebras $\mathcal{A}$ and $\mathcal{U}$, and the UDC associated with $\mathcal{A}$. As always, $\mathcal{U}$ is to be thought of as an algebra of left-invariant generalized derivations which act on elements of $\mathcal{A}$ in the manner described in Section 3.1. We now associate with each $x \in \mathcal{U}$ a new object, the Lie derivative $\mathcal{L}_{x}$; it is a 
linear function of $x$, has the same transformation properties as $x$ under $\mathcal{A}$-coactions (i.e. $\mathcal{L}_{x} \mapsto 1_{\mathcal{A}} \otimes \mathcal{L}_{x}$ and $\mathcal{L}_{x} \mapsto \mathcal{L}_{x^{(1)}} \otimes x^{(2)^{\prime}}$ ), and is a linear map taking $\Omega(\mathcal{A})$ into itself such that $p$-forms map to $p$-forms. Furthermore, we require that

$$
\mathcal{L}_{x} \delta=\delta \mathcal{L}_{x} .
$$

This relation allows us to uniquely recover the action of $\mathcal{L}_{x}$ on all of $\Omega(\mathcal{A})$ from its action on $\mathcal{A}$, i.e. 0 -forms. Just as in the classical case, the action of the Lie derivative on $a \in \mathcal{A}$ is defined to be the same as that of the corresponding differential operator, i.e.

$$
\mathcal{L}_{x}(a)=x \triangleright a=a_{(1)}\left\langle x, a_{(2)}\right\rangle,
$$

and its commutation relations with 0 -forms is the same as that given in $\mathcal{A} \rtimes \mathcal{U}$ :

$$
\mathcal{L}_{x} a=a_{(1)}\left\langle x_{(1)}, a_{(2)}\right\rangle \mathcal{L}_{x_{(2)}}=\mathcal{L}_{x_{(1)}}(a) \mathcal{L}_{x_{(2)}} .
$$

From (5.1) and (5.3) we can find the action on and commutation relation with any 1 -form $a \delta(b)$ :

$$
\begin{aligned}
\mathcal{L}_{x}(a \delta(b)) & =a_{(1)} \delta\left(b_{(1)}\right)\left\langle x, a_{(2)} b_{(2)}\right\rangle \\
\mathcal{L}_{x} a \delta(b) & =a_{(1)} \delta\left(b_{(1)}\right)\left\langle x_{(1)}, a_{(2)} b_{(2)}\right\rangle \mathcal{L}_{x_{(2)}}=\mathcal{L}_{x_{(1)}}(a \delta(b)) \mathcal{L}_{x_{(2)}} .
\end{aligned}
$$

At this point we introduce for each $x \in \mathcal{U}$ the corresponding inner derivation $i_{x}$. The guideline for this generalization of the classical case will be the classical Cartan identity

$$
\mathcal{L}_{x}=i_{x} \delta+\delta i_{x}
$$

(so $i_{x}$ is linear in $x$ ). To find the action of $i_{x}$ on $\Omega(\mathcal{A})$ we can now attempt to use (5.5) in the identity $\mathcal{L}_{x}(a)=i_{x}(\delta(a))+\delta\left(i_{x}(a)\right)$. We take as an assumption that the action of $i_{x}$ on 0 -forms like $a$ vanishes; therefore, we obtain

$$
i_{x}(\delta(a))=a_{(1)}\left\langle x, a_{(2)}\right\rangle .
$$

However, this cannot be true for any $x \in \mathcal{U}$ because $\delta(1)=0$. From $(5.6), i_{x}(\delta(1))=$ $1 \epsilon(x)$, which does not necessarily vanish identically (as we require). We see that the trouble arises when dealing with those $x \in \mathcal{U}$ with nonzero counit. This apparent inconsistency can be dealt with by noting that fof any $x$, the counit of $x-1_{\mathcal{U}} \epsilon(x)$ does vanish identically; thus, we modify equation (5.6) to read

$$
i_{x}(\delta(a))=a_{\{1)}\left\langle x-I_{\mathcal{U}} \epsilon(x), a_{(2)}\right\rangle,
$$


so that $i_{x}(\delta(1))$ does indeed vanish for all $x$. Also note that this requires the consistency condition

$$
i_{1 u} \equiv 0 \text {. }
$$

To define $\boldsymbol{i}_{x}$ for all $x \in \mathcal{U}$, therefore, we also need to modify equation (5.5) to

$$
\mathcal{L}_{x-1 u \epsilon(x)}=i_{x} \delta+\delta i_{x}
$$

or, in view of $(5.3)$, defining $\mathcal{L}_{1_{u}}:=\mathrm{id}$, and using the linearity of the Lie derivative,

$$
\mathcal{L}_{x}=i_{x} \delta+\delta i_{x}+\epsilon(x) \mathrm{id}
$$

(here id is the identity map on $\Omega(\mathcal{A})$, and therefore the unit in the algebra of generalized derivations, defined to be invariant under left- and right-coactions). We call this the universal Cartan identity. From this, it is apparent that $\mathcal{A}$ must coact on $i_{x}$ in the same way as on $\mathcal{L}_{x}$.

To find the complete commutation relations of $i_{x}$ with elements of $\Omega(\mathcal{A})$ rather than just its action on them, we need only determine how $i_{x}$ moves through 0 - and 1-forms. Both of these can be found by commuting $\mathcal{L}_{x}$ through a function $a \in \mathcal{A}$, using (5.3) and (5.10). The left-hand side of the former gives (using the Leibniz rule)

$$
\mathcal{L}_{x} a=i_{x} \delta(a)+i_{x} a \delta+\epsilon(x) a+\delta i_{x} a
$$

and the right-hand side gives

$$
\begin{aligned}
& a_{(1)}\left\langle x_{(1)}, a_{(2)}\right\rangle \mathcal{L}_{x_{(2)}}= \\
& \quad a_{(1)}\left\langle x_{(1)}, a_{(2)}\right\rangle \delta i_{x_{(2)}} \\
& \quad+a_{(1)}\left\langle x, a_{(2)}\right\rangle+a_{(1)}\left\langle x_{(1)}, a_{(2)}\right\rangle i_{x_{(2)}} \delta .
\end{aligned}
$$

Equating the two and using (C.2), (5.1), (5.7), and $i_{x}(a)=0$, we obtain

$$
i_{x} \delta(a)-i_{x}(\delta(a))+\mathcal{L}_{x_{(1)}}(\delta(a)) i_{x_{(2)}}=\left\{-i_{x} a+i_{x}(a)+\mathcal{L}_{x_{(1)}}(a) i_{x_{(2)}}, \delta\right\}
$$

Therefore, we propose the commutation relation

$$
i_{x} \phi=i_{x}(\phi)+(-1)^{p} \mathcal{L}_{x_{(1)}}(\phi) i_{x_{(2)}}
$$

for any $p$-form $\phi$, so that both sides of (5.13) vanish. 
Missing in our list are commutation relations of Lie derivatives with themselves and inner derivations. To find the $\mathcal{L}-\mathcal{L}$ relations, we the identity (4.8), and, as before, we extend the properties of the elements of $\mathcal{U}$ to those of the corresponding Lie derivatives. Therefore,

$$
\mathcal{L}_{x} \mathcal{L}_{y}=\mathcal{L}_{\left(x_{(1)}\right. \text { वd }} \mathcal{L}_{x_{(2)}},
$$

and therefore, using (5.10),

$$
\mathcal{L}_{x} i_{y}=i_{\left(x_{(1)}\right. \text { dd }} \mathcal{L}_{x_{(2)}} .
$$

(It would seem that $(5.10)$ could also give the relation

$$
i_{x} \mathcal{L}_{y}=\mathcal{L}_{\left(x_{(1)} \text { ad } y\right)} i_{x_{(2)}}+i_{\left(x-\mathcal{I}_{\mathcal{U}} \epsilon(x)\right) \triangleright(a d},
$$

but this is inconsistent with the commutation relation (5.14).)

After all these derivations (pun intended), it is probably convenient to pause for a while and recap our results from this section. Here is a summary of the actions of the Lie derivatives and inner derivations with 0 - and 1-forms:

$$
\begin{aligned}
\mathcal{L}_{x}(a) & =a_{(1)}\left\langle x, a_{(2)}\right\rangle, \\
\mathcal{L}_{x}(\delta(a)) & =\delta\left(a_{(1)}\right)\left\langle x, a_{(2)}\right\rangle, \\
\boldsymbol{i}_{x}(a) & =0 \\
\boldsymbol{i}_{x}(\delta(a)) & =a_{(1)}\left\langle x, a_{(2)}\right\rangle-\epsilon(x) a,
\end{aligned}
$$

where, as usual, $x \in \mathcal{U}, a \in \mathcal{A}$. These allow the actions of $\mathcal{L}$ and $i$ on an arbitrary $p$-form $\phi \in \Omega(\mathcal{A})$ to be found iteratively. Once this has been done, the commutation relations between the derivations and elements of $\Omega(\mathcal{A})$ are therefore

$$
\begin{aligned}
\mathcal{L}_{x} \phi & =\mathcal{L}_{x_{(1)}}(\phi) \mathcal{L}_{x_{(2)}} \\
\boldsymbol{i}_{x} \phi & =\boldsymbol{i}_{x}(\phi)+(-1)^{p} \mathcal{L}_{x_{(1)}}(\phi) \boldsymbol{i}_{x_{(2)}},
\end{aligned}
$$

(The actions and commutation relations for $\delta$ were already given when the UDC was introduced.) Finally, here are the relations between the derivations themselves:

$$
\begin{aligned}
& \{\boldsymbol{\delta}, \boldsymbol{\delta}\}=0, \\
& {\left[\delta, \mathcal{L}_{x}\right]=0,} \\
& \left\{\delta, i_{x}\right\}=\mathcal{L}_{x}-\epsilon(x) \text { id, } \\
& \mathcal{L}_{x} \mathcal{L}_{y}=\mathcal{L}_{\left(x_{(1) \triangleright} \text { ad }\right)} \mathcal{L}_{x_{(2)}} \\
& \mathcal{L}_{x} i_{y}=i_{\substack{\left(x_{(1)} \triangleright y\right) \\
\text { ad }}} \mathcal{L}_{x_{(2)}}
\end{aligned}
$$


Note that at this point we do not have $i-i$ commutation relations, which may at first seem a bit worrisome. However, this is not unexpected; $\boldsymbol{i}_{x} \boldsymbol{i}_{y}$ and $\boldsymbol{i}_{y} \boldsymbol{i}_{x}$ are simply elements of the calculus whose action on and commutation relations with $p$-forms are perfectly well-defined, in precisely the same way that $\delta(a) \delta(b)$ and $\delta(b) \delta(a)$ are simply elements of $\Omega(\mathcal{A})$. We have not assumed relations such as $\delta(a) \delta(b)+\delta(b) \delta(a) \equiv 0$ (unlike the "classical" case), so it is not surprising that we do not have any similar relations between the is. However, later in this chapter we will see that such restrictions between elements of $\Omega(\mathcal{A})$ may be imposed in some cases, and we will comment on the possibility of $\boldsymbol{i}-\boldsymbol{i}$ commutation relations.

\subsubsection{Cartan-Maurer Forms}

The most general left-invariant 1-form can be written [3]

$$
\omega_{a}:=S\left(a_{(1)}\right) \delta\left(a_{(2)}\right)=-\delta\left(S\left(a_{(1)}\right)\right) a_{(2)} ;
$$

we will refer to such an element of $\Omega(\mathcal{A})$ as the Cartan-Maurer form corresponding to the function $a \in \mathcal{A}$. This once again follows the familiar terminology: if $\mathcal{A}$ is an $m \times m$ matrix representation of some lie group with $\Delta(g)=g \dot{\otimes} g, S(g)=g^{-1}$ and $\epsilon(g)=I$ for $g \in \mathcal{A}$, then $\omega_{g}=g^{-1} \delta(g)$, i.e. $\omega_{g}$ is the well-known left-invariant classical Cartan-Maurer form. The exterior derivative of $\omega_{a}$ has a particularly nice form, given by

$$
\begin{aligned}
\delta\left(\omega_{a}\right) & =\delta\left(S\left(a_{(1)}\right)\right) \delta\left(a_{(2)}\right) \\
& =\delta\left(S\left(a_{(1)}\right)\right) a_{(2)} S\left(a_{(3)}\right) \delta\left(a_{(4)}\right) \\
& =-\omega_{a_{(1)}} \omega_{a_{(2)}} .
\end{aligned}
$$

The Lie derivative of $\omega_{a}$ is

$$
\begin{aligned}
\mathcal{L}_{x}\left(\omega_{a}\right) & =\mathcal{L}_{x_{(1)}}\left(S\left(a_{(1)}\right)\right) \mathcal{L}_{x_{(2)}}\left(\delta\left(a_{(2)}\right)\right) \\
& =\left\langle x_{(1)}, S\left(a_{(1)}\right)\right\rangle S\left(a_{(2)}\right) \delta\left(a_{(3)}\right)\left\langle x_{(2)}, a_{(4)}\right\rangle \\
& =\omega_{a_{(2)}}\left\langle x, S\left(a_{(1)}\right) a_{(3)}\right\rangle .
\end{aligned}
$$

The contraction of left-invariant forms with $\boldsymbol{i}_{x}$ gives a number in the field $k$, rather than a function in $\mathcal{A}$ (as was the case for $\delta(a)$ ):

$$
i_{x}\left(\omega_{a}\right)=-i_{x}\left(\delta\left(S\left(a_{(1)}\right)\right) a_{(2)}\right)
$$




$$
\begin{aligned}
& =-i_{x}\left(\delta\left(S\left(a_{(1)}\right)\right)\right) a_{(2)} \\
& =-\left\langle x-1 \mathcal{U} \epsilon(x), S\left(a_{(1)}\right)\right\rangle S\left(a_{(2)}\right) a_{(3)} \\
& =(-\langle x, S(a)\rangle+\epsilon(x) \epsilon(a)) 1 .
\end{aligned}
$$

As an exercise, as well as a demonstration of the consistency of our results, we will compute the same expression in a different way:

$$
\begin{aligned}
i_{x}\left(\omega_{a}\right) & =i_{x}\left(S\left(a_{(1)}\right) \delta\left(a_{(2)}\right)\right) \\
& =\left\langle x_{(1)}, S\left(a_{(1)}\right)\right\rangle S\left(a_{(2)}\right) i_{x_{(2)}}\left(\delta\left(a_{(2)}\right)\right) \\
& =\left\langle x_{(1)}, S\left(a_{(1)}\right)\right\rangle S\left(a_{(2)}\right) a_{(3)}\left\langle x_{(2)}-1_{\mathcal{U}} \epsilon\left(x_{(2)}\right), a_{(4)}\right\rangle \\
& =\left\langle x_{(1)}, S\left(a_{(1)}\right)\right\rangle\left\langle x_{(2)}-1_{\mathcal{U}} \epsilon\left(x_{(2)}\right), a_{(2)}\right\rangle 1 \\
& =(-\langle x, S(a)\rangle+\epsilon(x) \epsilon(a)) 1 .
\end{aligned}
$$

This result is a consequence of the fact that $\mathcal{U}$ was interpreted as an algebra of left-invariant differential operators, so $i_{x}\left(\omega_{a}\right)$ must be a left-invariant 0 -form, i.e. proportional to 1 .

As a final observation, if $\left\{e_{i}\right\}$ and $\left\{f^{i}\right\}$ are, respectively, (countable) bases of $\mathcal{U}$ and $\mathcal{A}$ with $\left\langle e_{i}, f^{j}\right\rangle=\delta_{i}^{j}$, the action of $\delta$ on functions $a \in \mathcal{A}$ may be expressed as

$$
\delta(a)=\mathcal{L}_{e_{i}}(a) \omega_{f^{i}}=-\omega_{S^{-1}}\left(f^{i}\right) \mathcal{L}_{e_{i}}(a)
$$

so that the Cartan-Maurer forms form a left-invariant basis for $\Gamma(\mathcal{A})$.

\subsubsection{General Cartan Calculus}

So far, we have only considered the case of the universal differential calculus of a Hopf algebra $\mathcal{A}$, as described in Appendix C.1, in which there is no à priori assumption of any commutation relations between 1-forms. However, in most cases which will appear in a physics context, we will want to consider situations in which there are such relations, i.e. the general differential calculus described in Appendix C.2. So the question is, how do we incorporate our Cartan calculus into this scheme? We start by assuming that we already have a general differential calculus on a Hopf algebra $\mathcal{A}$, and we define a subspace $\mathcal{T}_{\mathcal{M}} \subset \mathcal{U}$, given by

$$
\mathcal{T}_{\mathcal{M}}:=\{x \in \mathcal{U} \mid \epsilon(x)=0 ;\langle x, S(m)\rangle=0, m \in \mathcal{M}\}
$$

It is easily seen that the defining properties for $\mathcal{M}$ imply, respectively ${ }^{1}$,

\footnotetext{
${ }^{1}$ The converse is also true, i.e. we could start by defining $\mathcal{T}_{\mathcal{M}}$ as having the above properties, and taking $\mathcal{M}$ to be that subalgebra of $\mathcal{A}$ whose inner product with $S\left(\mathcal{T}_{\mathcal{M}}\right)$ vanishes.
} 
1. $1_{\mathcal{U}} \notin \mathcal{T}_{\mathcal{M}}$,

2. $\Delta\left(\mathcal{T}_{\mathcal{M}}\right) \subseteq \mathcal{U} \otimes\left(\mathcal{T}_{\mathcal{M}} \oplus 1_{\mathcal{U}}\right)$

3. $\mathcal{U}^{\mathrm{ad}} \mathcal{T}_{\mathcal{M}} \subseteq \mathcal{T}_{\mathcal{M}}$.

(These properties of $\mathcal{T}_{\mathcal{M}}$ should remind the reader of the definition of a QLA in Section 4.1. As we shall shortly see, this is not a coincidence.) Note that for $x \in \mathcal{T}_{\mathcal{M}}$ and $a \in \mathcal{A}$,

$$
i_{x}\left(\omega_{a}\right)=-\langle x, S(a)\rangle
$$

Suppose this vanishes; then either $x=0, a=1_{\mathcal{A}}$, or $a \in \mathcal{M}$. Therefore, if we restrict $a$ to be in $\mathcal{K} / \mathcal{M}$, then the vanishing of (5.28) implies that $x=0$ or $a=0$, i.e. the inner product $\langle\langle\rangle\rangle:, \mathcal{T}_{\mathcal{M}} \otimes \mathcal{K} / \mathcal{M} \rightarrow k$ defined by

$$
\langle\langle x, a\rangle\rangle:=-\langle x, S(a)\rangle
$$

is nondegenerate. Hence, $\mathcal{T}_{\mathcal{M}}$ and $\mathcal{K} / \mathcal{M}$ are dual to one another. The nondegeneracy of (5.29) guarantees that the map from $\mathcal{K} / \mathcal{M} \rightarrow \mathcal{T}_{\mathcal{M}}^{*}$ given by $a \mapsto \omega_{a}$ is bijective, insuring that $\Gamma_{\mathcal{M}}$ is the space of all 1-forms over $\mathcal{A}$. Therefore, to consistently define our Cartan calculus on all of $\Omega_{\mathcal{M}}$, we must restrict the arguments of the Lie derivative and inner derivation from $\mathcal{U}$ to $\mathcal{T}_{\mathcal{M}}$, and the argument of $\omega$ from $\mathcal{A}$ to $\mathcal{K} / \mathcal{M}$. As an example of how this works, note that for $x \in \mathcal{T}_{\mathcal{M}}$ and $a \omega_{m} \in \mathcal{N}_{\mathcal{M}}$,

$$
\begin{aligned}
\mathcal{L}_{x} a \omega_{m} & =a_{(1)} \omega_{m_{(2)}}\left\langle x_{(1)}, a_{(2)} S\left(m_{(1)}\right) m_{(3)}\right\rangle \mathcal{L}_{x_{(2)}} \\
\boldsymbol{i}_{x} a \omega_{m} & =-a_{(1)} \omega_{m_{(2)}}\left\langle x_{(1)}, a_{(2)} S\left(m_{(1)}\right) m_{(3)}\right\rangle i_{x_{(2)}} .
\end{aligned}
$$

Property (3) of $\mathcal{M}$ guarantees that $a_{(1)} \omega_{m_{(2)}}\left\langle x, a_{(2)} S\left(m_{(1)}\right) m_{(3)}\right\rangle \in \mathcal{N}_{\mathcal{M}}$ for all $x \in$ $\mathcal{U}$, so both sides of the two preceding equations are $\simeq 0$ in $\Gamma_{\mathcal{M}}$.

Note that we have not yet found a method for expressing any $\boldsymbol{i}-\boldsymbol{i}$ relations in a form depending manifestly on $\mathcal{M}$, i.e. in the manner of $\omega_{m_{(1)}} \omega_{m_{(2)}} \simeq 0$. However, in specific cases we can find such relations; this will be shown explicitly in the next chapter.

\subsection{Cartan Calculus for Quantum Lie Algebras}

If our Hopf algebra $\mathcal{U}$ is a $Q L A$, then the subspace $g$ satisfies precisely the same relations that $\mathcal{T}_{\mathcal{M}}$ does; this is of course the motivation for the definition of a QLA. 
Since the existence of the subspace $\mathcal{T}_{\mathcal{M}}$ implies the existence of the subalgebra $\mathcal{M}$ (and vice versa), we are dealing implicitly with the general, rather than the universal, case.

The first three of (5.20) look the same, and the second-to-last is simply (4.10) with the generators replaced by their corresponding Lie derivatives. The remaining commutation relation may be expressed using the explicit forms for the adjoint actions given in Section 4.1:

$$
\begin{aligned}
& \mathcal{L}_{A} i_{B}=i_{\chi_{A(1)} \text { ad } \chi_{B}} \mathcal{L}_{\chi_{A(2)}} \\
& =i_{\chi_{A} \triangleright \chi_{B}^{\text {ad }}} \mathcal{L}_{1_{\mathcal{H}}}+i_{\mathcal{O}_{A} D_{\square}^{\text {ad }} \chi_{B}} \mathcal{L}_{D} \\
& =f_{A B}{ }^{C} i_{C}+\hat{\mathbf{R}}^{C D}{ }_{A B} i_{C} \mathcal{L}_{D} \text {. }
\end{aligned}
$$

Once again, we see that in the $q \rightarrow 1$ limit, this becomes the familiar relation $\left[\mathcal{L}_{A}, i_{B}\right]=f_{A B}{ }^{C} i_{C}$.

\subsubsection{The Quasitriangular Case}

We now apply the results of the previous subsection to the case where our QLA is one derived from a quasitriangular Hopf algebra $\mathcal{U}$, with $\mathcal{A}$ being the dually paired Hopf algebra defined by a representation $\rho$ of $\mathcal{U}$ in the manner which the reader is certainly accustomed to by now.

We introduce the Lie derivative matrix $\mathcal{L}_{X}$ and inner derivation matrix $i_{X}$ as follows: $X$ is the matrix of elements of $\mathcal{U}$ defined by (4.30), and

$$
\left(\mathcal{L}_{X}\right)_{j}^{i}:=\mathcal{L}_{X^{i}{ }_{j}}, \quad\left(i_{X}\right)_{j}^{i}:=i_{X^{i}{ }_{j}} .
$$

These are of course related by the universal Cartan identity (5.10), i.e.

$$
\mathcal{L}_{X}=i_{X} \delta+\delta i_{X},
$$

where the term involving id does not appear because $\epsilon(X)=0$. The induced coactions of $\mathcal{A}$ on both these matrices are taken to be the same as those of $X$ itself.

The 0 -forms in $\Omega(\mathcal{A})$ are taken to be the elements of $\mathcal{A}$, as usual, and the basis for the 1 -forms are the elements of the matrix $\delta(A)$ (with coefficients in $\mathcal{A}$ ). However, as discussed previously, we will instead use the entries of the Cartan-Maurer matrix $\Omega$, given by

$$
\Omega^{i}{ }_{j}:=(S(A) \delta(A))_{j}^{i} .
$$


This matrix will figure prominently in the next chapter.

What do (5.20) look like in this formulation? The first four are just $\delta^{2}=0$, (5.33), $\delta \mathcal{L}_{X}=\mathcal{L}_{X} \delta$, and (4.31) with $X \rightarrow \mathcal{L}_{X}$, but by using the explicit forms of $\hat{\mathrm{R}}$ and the structure constants, the last can be written using the numerical R-matrix:

$$
R_{21} \mathcal{L}_{X_{1}} R i_{X_{2}}-i_{X_{2}} R_{21} \mathcal{L}_{X_{1}} R=\frac{1}{\lambda}\left(R_{21} R i_{X_{2}}-i_{X_{2}} R_{21} R\right)
$$

We also have the commutation relations with the 0 - and 1-forms:

$$
\begin{aligned}
\mathcal{L}_{X_{1}} A_{2} & =A_{2} R_{21} \mathcal{L}_{X_{1}} R+A_{2}\left(\frac{I-R_{21} R}{\lambda}\right), \\
R_{21} \mathcal{L}_{X_{1}} R \Omega_{2}-\Omega_{2} R_{21} \mathcal{L}_{X_{1}} R & =\frac{1}{\lambda}\left(R_{21} R \Omega_{2}-\Omega_{2} R_{21} R\right), \\
i_{X_{1}} A_{2} & =A_{2} R_{21} i_{X_{1}} R \\
R_{21} i_{X_{1}} R \Omega_{2}+\Omega_{2} R_{21} i_{X_{1}} R & =\frac{I-R_{21} R}{\lambda} .
\end{aligned}
$$

We can introduce another matrix of 1-forms, $\Omega^{\prime}$, which is defined in terms of the exterior derivative on 0 -forms:

$$
\delta(a) \equiv \operatorname{tr}\left(D^{-1} \Omega^{\prime} \mathcal{L}_{X}(a)\right),
$$

where $D$ is the numerical matrix defined in Appendix A.12. If we take $a$ as an entry of $A$, and require that the Leibniz rule holds, i.e.

$$
\operatorname{tr}\left(D^{-1} \Omega^{\prime} \mathcal{L}_{X}\right) A=\delta(A)+A \operatorname{tr}\left(D^{-1} \Omega^{\prime} \mathcal{L}_{X}\right)
$$

then by using the first of (5.36), we find that

$$
\begin{aligned}
\Omega_{1}^{\prime} A_{2} & =A_{2} R^{-1} \Omega_{1}^{\prime} R_{21}^{-1}, \\
\Omega & =\frac{1}{\lambda}\left[\operatorname{tr}_{1}\left(D_{1}^{-1} R^{-1} \Omega_{1}^{\prime} R_{21}^{-1}\right)-\operatorname{tr}\left(D^{-1} \Omega^{\prime}\right) I\right],
\end{aligned}
$$

Since the defining properties of a QLA are equivalent to starting with a general differential calculus, it is no surprise that we obtain these commutation relations between 0 - and 1-forms. Computing the entries of $\Omega$ in terms of those of $\Omega^{\prime}$ is of course immediate if we have the R-matrix; however, it may not always be possible to do the reverse, namely, to express these commutation relations in terms of the Cartan-Maurer forms rather than $\Omega^{\prime}$, since the second of the above equations may not be invertible. Whether or not this can be done will depend on the characteristic equation of the numerical R-matrix.

\footnotetext{
${ }^{2}$ We include the $D^{-1}$ in the trace so that $\Omega^{\prime}$ is left-invariant and right-covariant under the usual coactions.
} 


\section{Chapter 6}

\section{The Linear Quantum Groups $G L_{q}(N)$ and $S L_{q}(N)$}

\subsection{The Quantum Plane and the Quantum De- terminant}

In Appendix A.2.3, we defined the projectors associated with a given numerical $N^{2} \times N^{2}$-dimensional R-matrix. Now, we define the $N$-dimensional quantum hyperplane $[24,1,25]$ as follows: let $\left\{x^{i} \mid i=1, \ldots, N\right\}$ be coordinates and $\left\{d x^{i}\right\}$ be the associated differentials of a vector space on which the quantum group $\mathcal{A}$ associated with $\hat{R}$ coacts as

$$
x^{i} \mapsto A^{i}{ }_{j} \otimes x^{j}, \quad d x^{i} \mapsto A^{i}{ }_{j} \otimes d x^{j} .
$$

Furthermore, the quantum hyperplane is given a unital algebra structure by specifying commutation relations:

$$
\begin{aligned}
\left(P_{a}\right)^{i j}{ }_{k \ell} x^{k} x^{\ell} & =0, \quad a \in \mathcal{J}, \\
\left(P_{a}\right)_{k \ell}^{i j} d x^{k} d x^{\ell} & =0, \quad a \in \mathcal{J}^{\prime}
\end{aligned}
$$

where $\left\{P_{a} \mid a=1, \ldots, m\right\}$ are the projectors, and $\mathcal{J}$ and $\mathcal{J}^{\prime}$ are certain subsets of $\{1, \ldots, m\}$. Since $A$ satisfies (2.15), these commutation relations are consistent with the transformations (6.1). There must of course be further commutation relations between $x^{i}$ and $d x^{i}$, consistent not only with the above but also with the interpretation of $d x^{i}$ as the exterior derivative of $x^{i}$, but the form of these will depend on the characteristic equation of $\hat{R}$. 
Once the commutation relations between the differentials are specified, we can define $\epsilon_{q}$, the deformed version of the Levi-Civita tensor. This is done in the same way as in the undeformed case, i.e.

$$
d x^{i_{1}} d x^{i_{2}} \ldots d x^{i_{N}}=\epsilon_{q}^{i_{1} i_{2} \ldots i_{N}} d x^{1} d x^{2} \ldots d x^{N} .
$$

Once we have this, we can $\operatorname{define} \operatorname{det}_{q} A$, the quantum determinant of the matrix $A$, again in analogy with the classical case:

$$
A^{i_{1}}{ }_{j_{1}} \ldots A^{i_{N}}{ }_{j_{N}} \epsilon_{q}^{j_{1} \ldots j_{N}}=\epsilon_{q}^{i_{1} \ldots i_{N}} \operatorname{det}_{q} A .
$$

The cases we are most interested in in this chapter are $G L_{q}(N)$ and $S L_{q}(N)$; for these cases, there are the two projectors $P_{ \pm}$, and we take the coordinates to "commute" and their differentials to "anticommute", i.e.

$$
\left(P_{-}\right)^{i j}{ }_{k \ell} x^{k} x^{\ell}=\left(P_{+}\right)^{i j}{ }_{k \ell} d x^{k} d x^{\ell}=0 .
$$

In R-matrix notation, these commutation relations take the form

$$
\begin{aligned}
x^{j} x^{i} & =(r q)^{-1} R^{i j}{ }_{k \ell} x^{k} x^{\ell}, \\
d x^{j} d x^{i} & =-\frac{q}{r} R_{k \ell}^{i j} d x^{k} d x^{\ell} .
\end{aligned}
$$

The mixed commutation relation is then

$$
x^{j} d x^{i}=\frac{q}{r} R_{k \ell}^{i j} d x^{k} x^{\ell}
$$

This is obviously covariant under the coaction of $\mathcal{A}$, but it also respects the action of the exterior derivative; applying $d$ to this equation (with the Leibniz rule and $\left.d^{2}=0\right)$ just gives the second of $(6.6)$.

Now, we go ahead and use the $d x-d x$ commutation relation above to find $\epsilon_{q}$ from (6.3); note that since $r^{-1} \hat{R}$ is the same numerical matrix for both $G L_{q}(N)$ and $S L_{q}(N), \epsilon_{q}$ is independent of $r$, so it is the same for both $G L_{q}(N)$ and $S L_{q}(N)$. Furthermore, it satisfies the relations

$$
\begin{gathered}
\left(R_{0 N} \ldots R_{02} R_{01}\right)^{i_{0} i_{1} i_{2} \ldots i_{N}}{ }_{j_{0} j_{1} j_{2} \ldots j_{N}} \epsilon_{q}^{j_{1} j_{2} \ldots j_{N}}= \\
\left(R_{10} R_{20} \ldots R_{N 0}\right)^{i_{0} i_{1} i_{2} \ldots i_{N}}= \\
q r^{N} \delta_{j_{0} j_{1} \epsilon_{1} \epsilon_{2} \ldots j_{N}}^{i_{0} \epsilon_{q}^{i_{1} i_{2} \ldots j_{2} \ldots j_{N}}}=
\end{gathered}
$$


This relation implies an extremely important result which follows from (2.15), namely, $\operatorname{det}_{q} A$ commutes with all elements of $A$, and thus the entire Hopf algebra $\mathcal{A}$.

Note: This centrality of $\operatorname{det}_{g} A$ is by no means a result unique to $G L_{q}(N)$ and $S L_{q}(N)$; it turns out to be true for many other cases of interest as well. For example, for $S O_{q}(N)$, the commutation relations between the differentials are defined not only to be "antisymmetric", but also so that their contraction with the metric vanishes. In other words,

$$
\left(P_{1}\right)^{i j}{ }_{k \ell} d x^{k} d x^{\ell}=\left(P_{0}\right)^{i j}{ }_{k \ell} d x^{k} d x^{\ell}=0 .
$$

When we put this into $R$-matrix notation, we find that the commutation relations for the differentials take the form of the second of (6.6) with $r^{-1} R$ replaced by the $S O_{q}(N) \mathrm{R}$-matrix, and therefore (6.8) holds as well (with $q r^{N}$ replaced by 1 , that is). Thus, since (2.15) still holds, the centrality of the quantum determinant follows. It is this fact that allows us to give meaning to the " $S$ " in $S L_{q}(N), S O_{q}(N)$, and $S P_{q}\left(\frac{1}{2} N\right)$, since we could not interpret the matrices in these quantum groups as having unit determinant if it were not central. However, there are cases where $\operatorname{det}_{q} A$ is not central, most notably in multiparametric deformations such as $G L_{p q}(2)$ [26].

\subsubsection{The Cartan Calculus for $G L_{q}(N)$}

In order to apply the results of the previous section to the quantum group $G L_{q}(N)$, we first note that there does indeed exist a subalgebra $\mathcal{M}$ which satisfies the three criteria given in Appendix C.2, namely the one generated by the $N^{4}$ elements

$$
m_{k \ell}^{i j}:=\left(A_{1} A_{2}-A_{2}-R^{-1} A_{1} R_{21}^{-1}+R^{-1} R_{21}^{-1}\right)^{i j}{ }_{k \ell} .
$$

In checking that these elements generate $\mathcal{M}$, we must explicitly use the fact that the R-matrix for $G L_{q}(N)$ satisfies the quadratic characteristic equation

$$
\hat{R}^{2}-\lambda \hat{R}-I=0 \text {. }
$$

When we impose $\omega_{m} \simeq 0$ on these elements, we obtain the $A-\delta(A)$ commutation relations $[27,28,29,30]$

$$
\delta(A)_{1} A_{2}=R^{-1} A_{2} \delta(A)_{1} R_{21}^{-1} .
$$

Upon differentiation, the $\delta(A)-\delta(A)$ relations follow:

$$
\delta(A)_{1} \delta(A)_{2}+R^{-1} \delta(A)_{2} \delta(A)_{1} R_{21}^{-1}=0 .
$$


(Alternatively, we could have taken $\mathcal{M}$ as generated by

$$
m_{k \ell}^{i j}=\left(A_{1} A_{2}-A_{2}-R_{21} A_{1} R+R_{21} R\right)^{i j}{ }_{k \ell} .
$$

The resulting $A-\delta(A)$ commutation relations are

$$
A_{1} \delta(A)_{2}=R^{-1} \delta(A)_{2} A_{1} R_{21}^{-1}
$$

but the $\delta(A)-\delta(A)$ relations do not change.) The Cartan-Maurer matrix $\Omega=$ $S(A) \delta(A)$ therefore satisfies the following relations:

$$
\begin{aligned}
\Omega_{1} A_{2}-A_{2} R^{-1} \Omega_{1} R_{21}^{-1} & =0 \\
\Omega_{1} \delta(A)_{2}+\delta(A)_{2} R^{-1} \Omega_{1} R & =0 \\
\Omega_{1} R_{21}^{-1} \Omega_{2} R_{21}+R_{21}^{-1} \Omega_{2} R^{-1} \Omega_{1} & =0
\end{aligned}
$$

To relate $\Omega$ to $\Omega^{\prime}$ from the previous chapter, we merely use (6.11) and the Rmatrix trace relations from Appendix A.1. We find the simple relation $\Omega^{\prime}=-\alpha \Omega$, which of course is consistent with the commutation relations immediately above ${ }^{1}$.

We know from Appendix C.1.1 that the Cartan-Maurer forms are left-invariant and right-covariant, so the coactions of $G L_{q}(N)$ on $\Omega$ are the same as (4.33) with $X$ replaced by $\Omega$. Therefore, the 1 -form $\xi$ defined by taking the invariant trace of $\Omega$, namely

$$
\xi \equiv-\alpha \operatorname{tr}\left(D^{-1} \Omega\right)
$$

is left- and right-invariant. There is more than this to $\xi$, however; as a consequence of $(6.16),(6.11)$, and the various trace properties of $D$, we find

$$
\delta(A)=\lambda^{-1}[\xi, A], \quad \delta(\Omega)=-\Omega^{2}=\lambda^{-1}\{\xi, \Omega\},
$$

so $\xi$ is in fact the generator of the exterior derivative. These imply that the exterior derivative of any $p$-form $\phi$ is given by

$$
\delta(\phi)=\lambda^{-1}[\xi, \phi]_{ \pm}=\lambda^{-1}\left(\xi \phi-(-1)^{p} \phi \xi\right) .
$$

This may seem a bit weird, since in the classical case there is no such 1-form which generates the exterior derivative, but notice that the $\lambda$ in the above equation goes

\footnotetext{
${ }^{1}$ Note that in some treatments of this subject, such as [13], the 1-form matrix used is actually $\Omega^{\prime}$ and not $\Omega$.
} 
to zero in the classical limit. Since $\delta(\phi)$ still exists in this limit, this just implies that $\xi$ (anti)commutes with everything.

Now, we consider $\operatorname{det}_{q} A$; it is a 0 -form, and the above equations imply that

$$
\begin{array}{r}
\Omega \operatorname{det}_{q} A=q^{-2} \operatorname{det}_{q} A \Omega, \\
\delta\left(\operatorname{det}_{q} A\right)=-q^{-1} \operatorname{det}_{q} A \xi=-q \xi \operatorname{det}_{q} A .
\end{array}
$$

(A consequence of these equations is that both $\delta(\xi)$ and $\xi^{2}$ vanish.)

Now, we bring in the Lie derivatives and inner derivations, which we have already shown must satisfy (5.36) for any matrices $A$ and $\Omega$, including those from $G L_{q}(N)$. However, since we are now dealing with a specific Hopf algebra, with an R-matrix, commutation relations, and the works, it's no surprise that we have some more identities. For instance, by using (6.11), the combination $\frac{I-R_{21} R}{\lambda}$ could be replaced by $-\hat{R}$ if we wanted. In fact, this allows us to use the last of (5.36) to obtain the action of $i_{X}$ on the Cartan-Maurer matrix, which turns out to be

$$
i_{X_{1}}\left(\Omega_{2}\right)=-\alpha D_{2} P .
$$

In addition, it can be shown that $\xi$ and $\operatorname{det}_{q} A$ satisfy the following:

$$
\begin{aligned}
\mathcal{L}_{X} \xi=\xi \mathcal{L}_{X}, & i_{X} \xi+\xi i_{X}=I, \\
\mathcal{L}_{X} \operatorname{det}_{q} A=q^{2} \operatorname{det}_{q} A \mathcal{L}_{X}-q \operatorname{det}_{q} A, & i_{X} \operatorname{det}_{q} A=q^{2} \operatorname{det}_{q} A i_{X} .
\end{aligned}
$$

But perhaps the most meaningful results we obtain by considering a specific case are the commutation relations between the inner derivation matrices, as promised in the last chapter. They are reminiscent of the ones for $\Omega$, not surprisingly, and take the form

$$
R^{-1} i_{X_{1}} R i_{X_{2}}+i_{X_{2}} R_{21} i_{X_{1}} R=0 .
$$

Many of these relations take a much simpler form if we introduce a new matrix $\mathcal{Y}$, which corresponds to the matrix $Y$ from (4.21) in the same way $\mathcal{L}_{X}$ corresponds to $X$, i.e.

$$
\mathcal{Y}=\operatorname{Iid}-\lambda \mathcal{L}_{X}
$$

$\mathcal{Y}$ now is an operator within our differential calculus, and we obtain

$$
\begin{aligned}
\mathcal{Y} \delta & =\delta \mathcal{Y} \\
R_{21} \mathcal{Y}_{1} R i_{X_{2}} & =i_{X_{2}} R_{21} \mathcal{Y}_{1} R \\
R_{21} \mathcal{Y}_{1} R \mathcal{Y}_{2} & =\mathcal{Y}_{2} R_{21} \mathcal{Y}_{1} R \\
\mathcal{Y}_{1} A_{2} & =A_{2} R_{21} \mathcal{Y}_{1} R \\
R_{21} \mathcal{Y}_{1} R \Omega_{2} & =\Omega_{2} R_{21} \mathcal{Y}_{1} R
\end{aligned}
$$


as well as

$$
\mathcal{Y} \xi=\xi \mathcal{Y}, \quad \mathcal{Y} \operatorname{det}_{q} A=q^{2} \operatorname{det}_{q} A \mathcal{Y}
$$

However, $\mathcal{Y}$ is useful for more than making our equations prettier. Since its leading term is unity, it is invertible. More importantly, we can define a quantity Det $\mathcal{Y}$, which we identify as the determinant of $\mathcal{Y}$, satisfying

$$
\mathcal{Y} \operatorname{Det} \mathcal{Y}=\operatorname{Det} \mathcal{Y} \mathcal{Y}
$$

This quantity is defined through

$$
\left(\mathcal{Y}_{1 \ldots N}^{(1)} \ldots \mathcal{Y}_{1 \ldots N}^{(N)}\right)^{i_{1} \ldots i_{N}}{ }_{j_{1} \ldots j_{N}} \epsilon_{q}^{j_{1} \ldots j_{N}}=\epsilon_{q}^{i_{1} \ldots i_{N}} \operatorname{Det} \mathcal{Y}
$$

where

$$
\mathcal{Y}_{1 \ldots N}^{(k)}= \begin{cases}\left(R_{k N} \ldots R_{k(k+1)}\right)^{-1} \mathcal{Y}_{k}\left(R_{k N} \ldots R_{k(k+1)}\right) & \text { for } k=1, \ldots, N-1, \\ \mathcal{Y}_{N} & \text { for } k=N\end{cases}
$$

This determinant is invariant under transformations of $\mathcal{Y}$, and satisfies the following as a consequence of the above:

$$
\begin{aligned}
\delta \operatorname{Det} \mathcal{Y} & =\operatorname{Det} \mathcal{Y} \delta \\
\operatorname{Det} \mathcal{Y} i_{X} & =i_{X} \operatorname{Det} \mathcal{Y} \\
\operatorname{Det} \mathcal{Y} A & =q^{2} A \operatorname{Det} \mathcal{Y} \\
\operatorname{Det} \mathcal{Y} \Omega & =\Omega \operatorname{Det} \mathcal{Y} \\
\operatorname{Det} \mathcal{Y} \xi & =\xi \operatorname{Det} \mathcal{Y} \\
\operatorname{Det} \mathcal{Y} \operatorname{det}_{q} A & =q^{2 N} \operatorname{det}_{q} A \operatorname{Det} \mathcal{Y}
\end{aligned}
$$

The above equations for Det $\mathcal{Y}$ suggest the definition of an operator $\boldsymbol{H}_{\mathrm{o}}$ as

$$
\operatorname{Det} \mathcal{Y} \equiv q^{2 H_{\circ}}=I \text { id }+q \lambda\left[H_{\circ}\right]_{q} .
$$

$\boldsymbol{H}_{\mathrm{o}}$ defined in this way commutes with $\mathcal{Y}, \boldsymbol{\delta}, \boldsymbol{i}_{X}, \Omega$, and $\xi$, and satisfies

$$
\left[\boldsymbol{H}_{\mathrm{o}}, A\right]=A, \quad\left[\boldsymbol{H}_{\mathrm{o}}, \operatorname{det}_{q} A\right]=N\left(\operatorname{det}_{q} A\right) .
$$

This operator will be important in the next section. 


\section{$6.2 S L_{q}(N)$}

\subsubsection{The Quantum Group $S L_{q}(N)$}

There seems to be an obvious way to specify the quantum group $S L_{q}(N)$ : take the matrix $A$ and set its determinant to unity. This seems reasonable; $\operatorname{det}_{q} A$ is central, so within the context of the Hopf algebra, this would appear to be the right thing to do. Unfortunately, this doesn't work. True, $\operatorname{det}_{q} A$ commutes with the entries of $A$, but it does not commute with such quantities as $\Omega$ and $\mathcal{Y}$. Therefore, to restrict $G L_{q}(N)$ to $S L_{q}(N)$, instead of imposing $\operatorname{det}_{q} A=1_{\mathcal{A}}$ we define matrices $T$ as

$$
T=\left(\operatorname{det}_{q} A\right)^{-\frac{1}{N}} A .
$$

With $\operatorname{det}_{q} T$ defined as in (6.4), the centrality of $\operatorname{det}_{q} A$ automatically gives $T$ determinant unity. Furthermore, we also find that $\Delta(T)=T \dot{\otimes} T, \epsilon(T)=I$, and $S(T)=T^{\mathbf{- 1}}$. Therefore, this matrix $T$ is what we identify as an element of the defining representation of $S L_{q}(N)$, since it also satisfies (2.15) with $A$ replaced by $T$. However, as we will see in the next section, it becomes convenient to introduce the matrix

$$
\Re=q^{-\frac{1}{N}} R,
$$

which we identify as the R-matrix for $S L_{q}(N)$. Thus, we shall write (2.15) as

$$
\Re T_{1} T_{2}=T_{2} T_{1} \Re
$$

\subsubsection{The Calculus for $S L_{q}(N)$}

The exterior derivative on $S L_{q}(N)$ can be taken to be the same as that introduced on $G L_{q}(N)$; this is because $T$ is a function of elements of $A$, so its differentials are still given by

$$
\delta(T)=\lambda^{-1}[\xi, T] .
$$

Note that this implies that the Cartan-Maurer form $\tilde{\Omega}$ for $S L_{q}(N)$ is related to that of $G L_{q}(N)$ by

$$
\tilde{\Omega}:=S(T) \delta(T)=q^{\frac{2}{N}} \Omega+q\left[\frac{1}{N}\right]_{q} \xi .
$$

In the classical limit $q \rightarrow 1, \tilde{\Omega}$ is traceless, giving the appropriate reduction from $N^{2}$ to $N^{2}-1$ independent entries in $\tilde{\Omega}$, which of course agrees with the number 
of 1-forms of the classical group $S L(N)$. However, for $q \neq 1$, we have no such reduction, and we do indeed have $N^{2}$ linearly independent 1-forms for $S L_{q}(N)$.

We have thus found a way to set the determinant of our $S L_{q}(N)$ matrices to unity; for the calculus of the group, we must do something similar, namely impose a constraint so that the number of independent differential operators is reduced from $N^{2}$ to $N^{2}-1$. In a way, we have already done this, because (6.32) and (6.33) together imply $\left[\boldsymbol{H}_{0}, T\right]=0$, so that $\boldsymbol{H}_{\circ}$ commutes with everything of interest in $S L_{q}(N)$, i.e. matrices, forms, exterior derivative, etc. Thus, within the context

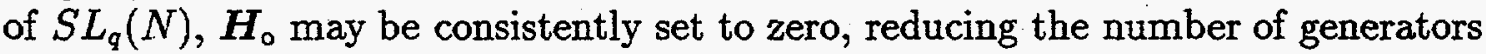
from $N^{2}$ to $N^{2}-1$, as desired. Explicitly, this restriction is accomplished by defining a new Lie derivative valued operator $\mathcal{Z}$ by $^{2}$

$$
\mathcal{Z}:=q^{-\frac{2}{2} \boldsymbol{H}_{0}} \mathcal{Y}
$$

Note that the determinant of $\mathcal{Z}$, computed using (6.28), is unity (or, to be more precise, id, which is the unit of the differential calculus). This is equivalent to the introduction of a set of $N^{2}$ "vector fields" $V^{i}{ }_{j}$ through $\mathcal{Z}=I$ id $-\lambda \mathcal{L}_{V}$, so that

$$
\mathcal{L}_{V}=\mathcal{L}_{X}+q^{-1}\left[\frac{\dot{\boldsymbol{H}}_{o}}{N}\right]_{q^{-1}} I-q^{-1} \lambda \mathcal{L}_{X}\left[\frac{\boldsymbol{H}_{o}}{N}\right]_{q^{-1}} .
$$

The fact that $\operatorname{Det} \mathcal{Z}=$ id implies that only $N^{2}-1$ of the elements of $\mathcal{L}_{V}$ are actually independent, which is precisely what we require for $S L_{q}(N)$. In the classical limit, $\boldsymbol{H}_{\mathrm{o}}=-\operatorname{tr}\left(\mathcal{L}_{X}\right)$, so $\mathcal{L}_{V}$ becomes traceless; thus, $V$ contains only $N^{2}-1$ linearly independent vector fields, as we'd expect.

Now that we have obtained all these quantities, we want to find the various relations they satisfy. As a starting point, note that the commutation relations between $\Omega$ and $T$ are given by

$$
\Omega_{1} T_{2}=q^{\frac{2}{N}} T_{2} R^{-1} \Omega_{1} R_{21}^{-1}=T_{2} \Re^{-1} \Omega_{1} \Re_{21}^{-1} .
$$

Here we see the appearance of the $S L_{q}(N) R$-matrix $\Re$, as promised. In fact, there is a general pattern: by using the substitutions $A \rightarrow T, R \rightarrow \Re$, and $\mathcal{L}_{X} \rightarrow \mathcal{L}_{V}$, we obtain most of the corresponding relations for $S L_{q}(N)$. This only goes so far, though; we do not generally make the substitution $\Omega \rightarrow \tilde{\Omega}$ or $i_{X} \rightarrow i_{V}$. This would seem to be a result of the fact that many of the relations satisfied by these quantities

\footnotetext{
${ }^{2}$ When restricted to acting on 0 -forms, this operator is identical to the operator $Y$ in [13].
} 
arise as a result of going from the universal differential calculus to the general. In any case, $\mathcal{L}_{V}$ satisfies

$$
\begin{aligned}
\Re_{21} \mathcal{L}_{V_{1}} \Re \mathcal{L}_{V_{2}}-\mathcal{L}_{V_{2}} \Re{ }_{21} \mathcal{L}_{V_{1}} \Re=\lambda^{-1}\left(\Re_{21} \Re \mathcal{L}_{V_{2}}-\mathcal{L}_{V_{2}} \Re{ }_{21} \Re\right), \\
\Re_{21} \mathcal{L}_{V_{1}} \Re i_{X_{2}}-i_{X_{2}} \Re{ }_{21} \mathcal{L}_{V_{1}} \Re=\lambda^{-1}\left(\Re_{21} \Re i_{X_{2}}-i_{X_{2}} \Re \Re_{21} \Re\right),
\end{aligned}
$$

and we also find the relations between our new differential operators and the 0 - and 1-forms of $S L_{q}(N)$ :

$$
\begin{aligned}
\mathcal{L}_{V_{1}} T_{2} & =T_{2} \Re_{21} \mathcal{L}_{V_{1}} \Re+T_{2}\left(\frac{I-\Re_{21} \Re}{\lambda}\right), \\
\Re_{21} \mathcal{L}_{V_{1}} \Re \Omega_{2}-\Omega_{2} \Re_{21} \mathcal{L}_{V_{1}} \Re & =\lambda^{-1}\left(\Re_{21} \Re \Omega_{2}-\Omega_{2} \Re{ }_{21} \Re\right), \\
i_{X_{1}} T_{2} & =T_{2} \Re_{21} i_{X_{1}} \Re, \\
\Re_{21} i_{X_{1}} \Re \tilde{\Omega}_{2}+\tilde{\Omega}_{2} \Re_{21} i_{X_{1}} \Re & =\frac{I-\Re_{21} \Re}{\lambda} .
\end{aligned}
$$

Since $\delta$ still commutes with $\mathcal{L}_{V}$ and is still generated by $\xi$, we obviously also have

$$
\mathcal{L}_{V} \xi=\xi \mathcal{L}_{V} .
$$

The relations for $\mathcal{Z}$ corresponding to (6.25) are easily obtained by using $\mathcal{L}_{V}=$ $\frac{\text { Iid }-\mathcal{Z}}{\lambda}$ in all of the above equations. 


\section{Chapter 7}

\section{Conclusions}

In this work, we have presented a general approach to the analysis of the differential geometry of Hopf algebras and quantum groups, primarily through the introduction of the Lie algebra of derivations and the resulting Cartan calculus. The concentration has been on those cases which often arise in physical problems, namely, the deformed versions of the groups $G L(N)$ and $S L(N)$, although we have tried to develop methods and approaches which will be useful for other cases as well. In this we have been largely successful, but there are still several avenues which have not been dealt with entirely, and in this final chapter, we will address some of them.

\subsection{The Killing Metric}

In Chapter 4.4, the Killing form for an arbirtary quasitriangular Hopf algebra was introduced. When the Hopf algebra was also a QLA, we could define the deformed version of the Killing metric, which we found had many of the same properties and uses as the classical one. However, there were also hints that it had more in common with the classical Killing metric than was first thought. The appearance of the same $3 \times 3$ submatrix for the fundamental and adjoint representations of $S L_{q}(2)$ (up to a factor) was immediately reminiscent of the classical case, where the Killing metric of an irreducible representation of a compact Lie algebra is proportional to some canonical form. This similarity was even more suggested by the fact that the quadratic Casimirs for these two representations had eigenvalues whose ratio had the correct classical limit.

All this evidence seems to point to the possibility that most, or perhaps even all, 
of the properties of the classical Killing metric have analogues in the deformed case. There may indeed exist some canonical form of the Killing metric; if so, this would allow the definition of the level of a representation as the proportionality constant between its Killing metric and the canonical one. The classification of irreducible representations of QLAs by the values of their deformed quadratic Casimirs also seems to be a distinct possibility. Many of the objects in classical physics depend on such representation-dependent quantities (e.g. the QCD $\beta$-function's dependence on the $S U\left(N_{f}\right)$ Casimir), so studying the properties of the Killing metric may be extremely fruitful in the context of a deformed field theory.

\subsection{Inner Derivations for a General Cartan Cal- culus}

When we developed the Cartan calculus in Chapter 5, the initial lack of any $\boldsymbol{i}-\boldsymbol{i}$ commutation relations was not a surprise; after all, we were dealing purely with the universal differential calculus, which was itself missing any commutation relations between 1-forms. Since the inner derivations and Cartan-Maurer forms are in a sense dual to each other (i.e. $i_{x}\left(\omega_{a}\right)$ is proportional to the unit), this was expected.

Unfortunately, the same cannot be said for the general differential calculus. There we do in fact have commutation relations between 0 - and 1 -forms, given by the vanishing of the Cartan-Maurer form on the subalgebra $\mathcal{M}$. We even have the dual version of this condition, the existence of the subspace $\mathcal{T}_{\mathcal{M}}$. It was shown that the previously existing Cartan calculus could easily accomodate the general differential calculus, by restricting the arguments of the Lie derivative and the inner derivation to $\mathcal{T}_{\mathcal{M}}$, but it should also follow that there are some sort of $i-i$ relations dual to the $\omega-\omega$ relations in $\Omega_{\mathcal{M}}$. Such (anti)commutation relations have yet to be found, and this remains one of the glaring faults in the treatment of the Cartan calculus in this work.

\section{3 $S O_{q}(N)$ and $S P_{q}\left(\frac{1}{2} N\right)$}

In Chapter 6, we considered the application of the results of Chapter 5 to the specific cases of $G L_{q}(N)$ and $S L_{q}(N)$, by using the fact that we knew the numerical $\mathrm{R}$-matrix explicitly, and could find the subalgebra $\mathcal{M}$ needed to restrict from the universal differential calculus to the general, from (6.10). We also found that there 
were consistent sets of equations, still given in terms of $R$, which gave the deformed anticommutation relations between the inner derivations. The differential geometry of $S L_{q}(N)$ was then shown to be obtainable from that of $G L_{q}(N)$ via the restriction (6.33).

Our success in doing so begs the question: can we do the same, or something similar, with the other quantum Lie algebras $S O_{q}(N)$ and $S P_{q}\left(\frac{1}{2} N\right)$ ? There are certainly still some relations which will hold for the differential calculus of all quasitriangular Lie algebras, namely those discussed in Chapter5.2.1. For instance, the construction of the smash product for the quasitriangular case was completely general, so one may introduce the differential operators $X$ and therefore the 1-forms $\Omega^{\prime}$ via the exterior derivative (5.37); this was done explicitly (albeit in somewhat different notation) in [31]. In fact, since the characteristic equations for $S O_{q}(N)$ and $S P_{q}\left(\frac{1}{2} N\right)$ are explicitly known, we can even find the relation between $\Omega$ and $\Omega^{\prime}$ given in (5.39):

$$
\Omega^{i}{ }_{j}=\left(\hat{R}^{-1}-q^{\epsilon-N} I\right)^{\ell n}{ }_{k j} g^{i k} g_{\ell m} \Omega^{\prime m}{ }_{n} .
$$

However, this only goes so far; recall that, in the fundamental representation, the quantum matrices $A$ for these quantum groups are not only restricted by a determinant condition, but also the metric condition

$$
g^{k \ell} A^{i}{ }_{k} A_{\ell}^{j}=g^{i j}
$$

where $g^{i j}$ is the appropriate numerical matrix from Appendix A.2. Consistency between this and (2.15) implies the numerical relations

$$
R^{i j}{ }_{k \ell}=g^{i m} \tilde{R}_{m \ell}^{n j} g_{n k}=g^{j m}\left(R^{-1}\right)^{i n}{ }_{k m} g_{n \ell},
$$

and these, together with (3.7), imply similar conditions on $L^{ \pm}$:

$$
g^{k \ell}\left(L^{ \pm}\right)_{\ell}^{j}\left(L^{ \pm}\right)_{k}^{i}=g^{i j}
$$

In terms of the matrix $Y:=L^{+} S\left(L^{-}\right)$, this translates into the condition

$$
\left(\hat{R}^{-1} Y_{2} \hat{R} Y_{2}\right)^{i j}{ }_{k \ell} g^{k \ell}=g^{i j} \text {. }
$$

Is it possible to start from the $G L_{q}(N)$ case, which we know well, and impose (7.2) and (7.5) somehow? The answer would seem to be no, since there is no obvious way to construct an $A$ satisfying (7.2) from a general $G L_{q}(N)$ matrix. Finding the differential geometry on these groups may therefore only be tractable by starting from scratch, by determining what subalgebra $\mathcal{M}$ not only satisfies the three criteria in Appendix C.2, but also respects the metric condition. At this point, such a subalgebra has not been found (at least not to our knowledge), so this remains an open problem. 


\subsection{Fiber Bundles and Deformed Gauge Theo- ries}

Finally, we reconsider the main motivation behind this work, namely, the formulation of a deformed gauge field theory. As alluded to in the Introduction, the main reason for including Appendix B in this work was not only to remind the reader of what the classical Cartan calculus is, but also to present the general method by which topological properties (such as continuity of a function) are related to algebraic concepts (e.g. the unital associative algebra Fun $(M)$ ). At least in the case where the manifold in question is a topological group, and therefore the corresponding function algebra a quantum group, we have largely succeeded, with the linear cases $G / S L_{q}(N)$ being the most fully realized. If we also limit ourselves to deformations of principal bundles, we are even closer to our goal, because then the entire bundle (as well as the structure group) is described by a Hopf algebra. Others have already had much success in this case [32,33], and this is generally the one of interest for most physical systems.

What remains is to consider the cases where the bundle is not principle. The structure group continues to be treated as a Hopf algebra, so the results herein still apply, but the base manifold and fiber are treated as unital associative algebras (or more precisely, collections of unital associative algebras, together with transition functions relating each algebra, $\grave{a}$ la sheaf theory). How our conclusions can be applied to these cases is as yet unknown, but there seems to be no reason to assume that our techniques would be utterly useless, so we are still (perhaps naively) optimistic that deformed gauge theories are within reach. 


\section{Bibliography}

[1] J. Wess and B. Zumino, Nucl. Phys. B (Proc. Suppl.) 18302 (1990)

[2] A. Connes, Pub. Math. (IHES) 62257 (1985)

[3] S. L. Woronowicz, Commun. Math. Phys. 122125 (1989)

[4] N. Yu. Reshetikhin, L. A. Takhtadzhyan and L. D. Faddeev, Leningrad Math. J. $1193(1990)$

[5] M. E. Sweedler, Hopf Algebras, Benjamin Press, 1969

[6] E. Abe, Hopf Algebras, Cambridge University Press, 1977

[7] S. Majid, Int. J. Mod. Phys. A 51 (1990)

[8] V. G. Drinfel'd, in: Proc. Int. Congr. Math., Berkeley 1985 (1986)

[9] S. L. Woronowicz, Commun. Math. Phys. 111613 (1987)

[10] M. Jimbo, Int. J. Mod. Phys. A 43759 (1989)

[11] V. G. Drinfel'd, Sov. Math. Dokl. 32254 (1985)

[12] C. Chryssomalakos, private communication

[13] B. Zumino, in: K. Schmüdgen (ed.), Math. Phys. X, Proc. $X^{\text {th }}$ IAMP Conf., Leipzig 1991, Springer-Verlag, 1992

[14] S. Majid, Int. J. Mod. Phys. A 84521 (1993)

[15] S. L. Woronowicz, preprint "Quantum $E(2)$ Group and its Pontryagin Dual" 
[16] M. Schlieker, W. Weich and R. Weixler, Z. Phys. C 5379 (1992)

[17] L. Castellani, Phys. Lett. B 298335 (1993)

[18] M. Rosso, Commun. Math. Phys. 124307 (1989)

[19] E. Celeghini, R. Giachetti, E. Sorace and M. Tarlini, J. Math. Phys. 312548 (1990)

[20] D. Bernard, Prog. Theor. Phys. Supp. 10249 (1990)

[21] B. Jurčo, Lett. Math. Phys. 22177 (1991)

[22] N. Yu. Reshetikhin and M. A. Semenov-Tian-Shansky, Lett. Math. Phys. 19 $133(1990)$

[23] B. Zumino, private communication

[24] Yu. Manin, Commun. Math. Phys. 123163 (1989)

[25] B. Zumino, Mod. Phys. Lett. A 131225 (1991)

[26] A. Schirrmacher, J. Wess and B. Zumino, Z. Phys. C 49317 (1991)

[27] Yu. Manin, Bonn preprint MPI/91-47 (1991)

Yu. Manin, Bonn preprint MPI/91-60 (1991)

[28] G. Maltsiniotis, C. R. Acad. Sci. Paris 331831 (1990)

G. Maltsiniotis, ENS preprint "Calcul Differentiel sur le Groupe Linéaire Quantique" (1990)

[29] A. Schirrmacher, in: Proc. $1^{\text {st }}$ Max Born Symp. Theor. Phys., Wroclaw 1991

[30] A. Sudbery, York preprint PRINT-91-0498 (1991)

A. Sudbery, Phys. Lett. B 28461 (1992)

[31] U. Carow-Watamura, M. Schlieker, S. Watamura and W. Weich, Commun. Math. Phys. 142605 (1991)

[32] T. Brzeziński and S. Majid, Commun. Math. Phys. 157591 (1993)

[33] M. J. Pflaum, preprint GK-MP-9403/1 (1994)

M. J. Pflaum and P. Schauenburg, preprint GK-MP-9407/7 (1994) 
[34] B. Schutz, Geometrical Methods of Mathematical Physics, Cambridge University Press, 1980

[35] F. W. Warner, Foundations of Differentiable Manifolds and Lie Groups; Springer-Verlag, 1983

[36] M. Göckeler and T. Schücker, Differential Geometry, Gauge Theories, and Gravity, Cambridge University Press, 1987

[37] R. Coquereaux, preprint CPT-88/P-2147 (1988)

[38] M. Schlieker and B. Zumino, preprint LBL-35299, UCB-PTH-94/03, hep-th 9403093 (1994) 


\section{Appendix A}

\section{Numerical R-Matrix Relations}

\section{A.1 The Element $u$}

Suppose $\mathcal{U}$ is a quasitriangular Hopf algebra with universal $\mathrm{R}$-matrix $\mathcal{R}$; then there exists an invertible element $u$ defined by [8]

$$
u:=m\left((S \otimes \mathrm{id})\left(\mathcal{R}_{21}\right)\right)=S\left(r^{\alpha}\right) r_{\alpha} .
$$

$u$ has counit $1_{k}$, and its inverse and coproduct are

$$
\begin{aligned}
u^{-1} & =r^{\alpha} S^{2}\left(r_{\alpha}\right) \\
\Delta(u) & =\left(\mathcal{R}_{21} \mathcal{R}\right)^{-1}(u \otimes u)=(u \otimes u)\left(\mathcal{R}_{21} \mathcal{R}\right)^{-1} .
\end{aligned}
$$

This element generates the square of the antipode via an inner automorphism:

$$
S^{2}(x)=u x u^{-1}
$$

for all $x \in \mathcal{U}$. A consequence of this is that the element $c:=u S(u)$ is central in $\mathcal{U}$.

Suppose we have a faithful $N \times N$ matrix representation $\rho$ on $\mathcal{U}$, and $A$ is the associated matrix of dual elements in $\mathcal{A}$ (see Chapter 2.3). We define the numerical matrix $D$ to be equal to $u$ in this representation, up to an overall multiplicative constant $\alpha$ :

$$
D^{i}{ }_{j}:=\alpha\left\langle u, A^{i}{ }_{j}\right\rangle .
$$

Several results follow immediately: first of all, an explicit computation using the definition of $u$ leads to the result

$$
I=\alpha \operatorname{tr}_{1}\left(D_{1}^{-1} \hat{R}^{-1}\right)=\alpha^{-1} \operatorname{tr}_{2}\left(D_{2} \hat{R}\right),
$$


where $\operatorname{tr}_{J}$ is shorthand for the contraction over the $J$ th pair of indices, e.g. the ${ }_{j}^{i}$ th element of the rightmost expression in the above equation is $\alpha^{-1} D^{m}{ }_{n} \hat{R}^{i n}{ }_{j m}$. These relations can be "inverted" in the sense of solving them for $D$ and $D^{-1}$; to do this, we introduce for any $N^{2} \times N^{2}$ matrix $K$ a matrix $\tilde{K}=\left[\left(K^{t_{1}}\right)^{-1}\right]^{t_{1}}\left(t_{J}\right.$ denotes transposing with respect to the $J$ th pair of indices). When this matrix exists, it satisfies

$$
K^{i m}{ }_{n \ell} \tilde{K}^{n k}{ }_{j m}=K_{\ell n}^{m i} \tilde{K}_{m j}^{k n}=\delta_{j}^{i} \delta_{\ell}^{k} .
$$

With this in hand, we find

$$
D=\alpha \operatorname{tr}_{2}(P \tilde{R}), \quad D^{-1}=\alpha^{-1} \operatorname{tr}_{2}\left(P\left(\tilde{R^{-1}}\right)\right) .
$$

Since the representation is faithful (by assumption), $c$ must be proportional to the unit matrix in the representation. We therefore define the constant $\beta$ by means of the identity

$$
\left\langle c, A_{j}^{i}\right\rangle=(\alpha \beta)^{-1} \delta_{j}^{i} .
$$

Using the explicit forms of $c$ and $u$ gives

$$
I=\beta^{-1} \operatorname{tr}_{1}\left(D_{1}^{-1} \hat{R}\right)=\beta \operatorname{tr}_{2}\left(D_{2} \hat{R}^{-1}\right),
$$

or, if we "invert",

$$
D=\beta^{-1} \operatorname{tr}_{1}\left(P\left(\tilde{R^{-1}}\right)\right), \quad D^{-1}=\beta \operatorname{tr}_{1}(P \tilde{R}) .
$$

From the fact that $\left(S^{2} \otimes S^{2}\right)(\mathcal{R})=\mathcal{R}$, we find the numerical relation

$$
D_{1} D_{2} R=R D_{1} D_{2} .
$$

The dual version in $\mathcal{A}$ of (A.3) is

$$
S^{2}(A)=D A D^{-1} .
$$

The definition of the $D$-matrix, together with (2.15), gives

$$
\left(D^{-1}\right)^{t} A^{t} D^{t} S(A)^{t}=S(A)^{t}\left(D^{-1}\right)^{t} A^{t} D^{t}=1_{\mathcal{A}},
$$

(2.15) and (A.13) together then imply the identities

$$
\tilde{R}=D_{1}^{-1} R^{-1} D_{1}=D_{2} R^{-1} D_{2}^{-1} .
$$


All of the above give the following important results: if $M$ is an $N \times N$ matrix, then

$$
\begin{aligned}
\operatorname{tr}_{1}\left(D_{1}^{-1} R^{-1} M_{1} R\right)^{i}{ }_{j} & =\operatorname{tr}_{1}\left(D_{1}^{-1} R_{21} M_{1} R_{21}^{-1}\right)_{j}^{i} \\
& =\operatorname{tr}\left(D^{-1} M\right) \delta_{j}^{i} .
\end{aligned}
$$

Also, if the elements of $M$ commute with the elements of $A$,

$$
\operatorname{tr}\left(D^{-1} S(A) M A\right)=\operatorname{tr}\left(D^{-1} M\right)
$$

In particular, if $M$ is a matrix on which $\mathcal{A}$ right coacts via $\Delta_{\mathcal{A}}\left(M^{i}{ }_{j}\right)=M_{\ell}^{k} \otimes$ $S\left(A^{i}{ }_{k}\right) A^{\ell}{ }_{j}$, then (A.13) implies

$$
\Delta_{\mathcal{A}}\left(\operatorname{tr}\left(D^{-1} M\right)\right)=\operatorname{tr}\left(D^{-1} M\right) \otimes 1_{\mathcal{A}}
$$

For this reason, $\operatorname{tr}\left(D^{-1} M\right)$ is called the invariant trace of $M$.

\section{A.2 R-Matrices for the Simple Lie Algebras}

In their seminal work [4], Reshetikhin, Takhtadzhyan and Faddeev give the numerical $\mathrm{R}$-matrices for the quantum versions of the fundamental representations of the Lie algebras $A_{n}, B_{n}, C_{n}$ and $D_{n}$; here, we review these forms, and include other results for the particular cases.

\section{A.2.1 R- and D-Matrices}

We take $E_{I J}$ to be the $N \times N$ numerical matrix whose only nonzero entry is a 1 at $(I, J)$. Furthermore, the tensor product which appears is that between numerical spaces; specifically, the $N^{2} \times N^{2}$-dimensional matrix $E_{I J} \otimes E_{K L}$ has entries

$$
\left(E_{I J} \otimes E_{K L}\right)^{i j}{ }_{k \ell}=\delta_{I}^{i} \delta_{J}^{j} \delta_{k}^{K} \delta_{\ell}^{L}
$$

The $D$ matrices take the standard diagonal form

$$
D=\sum_{I} q^{-2 \rho_{I}} E_{I I}
$$

where the $N$ values of $\rho_{I}$ will be given in each case. Where primed indices appear, they are defined to be $I^{\prime}=N+1-I$. 
1. $A_{n}=S L_{q}(n+1=N)$ :

$$
\begin{aligned}
R & =q^{-\frac{1}{N}}\left(q \sum_{I} E_{I I} \otimes E_{I I}+\sum_{I \neq J} E_{I I} \otimes E_{J J}+\lambda \sum_{I>J} E_{I J} \otimes E_{J I}\right), \\
\left(\rho_{1}, \ldots, \rho_{N}\right) & =(0,-1, \ldots,-n) .
\end{aligned}
$$

2. $B_{n}=S P_{q}\left(n=\frac{1}{2} N\right)$ :

$$
\begin{aligned}
R= & \sum_{I}\left(q E_{I I} \otimes E_{I I}+q^{-1} E_{I^{\prime} I^{\prime}} \otimes E_{I I}\right)+\sum_{I \neq J, J^{\prime}} E_{I I} \otimes E_{J J} \\
& +\lambda \sum_{I>J}\left(E_{I J} \otimes E_{J I}-q^{\rho_{I}-\rho_{J}} \epsilon_{I} \epsilon_{J} E_{I J} \otimes E_{I^{\prime} J^{\prime}}\right), \\
\left(\rho_{1}, \ldots, \rho_{N}\right)= & (n, n-1, \ldots, 1,-1, \ldots,-(n-1),-n), \\
\epsilon_{I}= & \begin{cases}+1 & I=1, \ldots, n, \\
-1 & I=(n+1), \ldots, N .\end{cases}
\end{aligned}
$$

3. $C_{n}=S O_{q}(2 n+1=N)$ :

$$
\begin{aligned}
R= & \sum_{I \neq n+1}\left(q E_{I I} \otimes E_{I I}+q^{-1} E_{I^{\prime} I^{\prime}} \otimes E_{I I}\right)+E_{n+1, n+1} \otimes E_{n+1, n+1} \\
& +\sum_{I \neq J, J^{\prime}} E_{I I} \otimes E_{J J}+\lambda \sum_{I>J}\left(E_{I J} \otimes E_{J I}-q^{\rho_{I}-\rho_{J}} E_{I J} \otimes E_{I^{\prime} J^{\prime}}\right), \\
\left(\rho_{1}, \ldots, \rho_{N}\right)= & \left(n-\frac{1}{2}, n-\frac{3}{2}, \ldots, \frac{1}{2}, 0,-\frac{1}{2}, \ldots,-\left(n-\frac{3}{2}\right),-\left(n-\frac{1}{2}\right)\right) .
\end{aligned}
$$

4. $D_{n}=S O_{q}(2 n=N)$ :

$$
\begin{aligned}
R= & \sum_{I}\left(q E_{I I} \otimes E_{I I}+q^{-1} E_{I^{\prime} I^{\prime}} \otimes E_{I I}\right)+\sum_{I \neq J, J^{\prime}} E_{I I} \otimes E_{J J} \\
& +\lambda \sum_{I>J}\left(E_{I J} \otimes E_{J I}-q^{\rho_{I}-\rho_{J}} E_{I J} \otimes E_{I^{\prime} J^{\prime}}\right), \\
\left(\rho_{1}, \ldots, \rho_{N}\right)= & (n-1, n-2 \ldots, 1,0,0,1, \ldots,-(n-2),-(n-1)) .
\end{aligned}
$$

\section{A.2.2 Characteristic Equations and Trace Relations}

The matrices $\hat{R}$ satisfy certain characteristic equations; in the context of knot theory, these are viewed as skein relations, relating particular sequences of strand crossings. 
For the deformed Lie algebras we consider here, these characteristic equations are of two types: the R-matrices of $G L_{q}(N)$ and $S L_{q}(N)$ satisfy the quadratic equation

$$
(\hat{R}-r q I)\left(\hat{R}+r q^{-1} I\right)=0,
$$

where $r=1$ for $G L_{q}(N)$ and $r=q^{-\frac{1}{N}}$ for $S L_{q}(N)$. The R-matrices for $S O_{q}(N)$ and $S P_{q}\left(\frac{1}{2} N\right)$, on the other hand, satisfy the cubic equation

$$
(\hat{R}-q I)\left(\hat{R}+q^{-1} I\right)\left(\hat{R}-\epsilon q^{\epsilon-N} I\right)=0,
$$

where $\epsilon=+1$ for $S O_{q}(N)$ and $\epsilon=-1$ for $S P_{q}\left(\frac{1}{2} N\right)$.

Now, we have all the trace relations discussed in the first section of this appendix; since the $\mathrm{R}$ - and $\mathrm{D}$-matrices have been given, we are now in a position to compute the constants $\alpha$ and $\beta$, as well at the trace of $D$ itself. Just like the characteristic equations, these computations split up into two types: for $G L_{q}(N)$ and $S L_{q}(N)$, we find

$$
\alpha=r q^{2 N-1}, \quad \beta=r q, \quad \operatorname{tr} D=\frac{q^{2 N}-1}{q^{2}-1},
$$

and for $S O_{q}(N)$ and $S P_{q}\left(\frac{1}{2} N\right)$,

$$
\alpha=\beta=q^{N-\epsilon}, \quad \operatorname{tr} D=\frac{q^{N-\epsilon}-q^{-(N-\epsilon)}}{q-q^{-1}}+\epsilon .
$$

( $\operatorname{tr} D^{-1}$ can be obtained simply by replacing $q$ with $q^{-1}$ in the expression for $\operatorname{tr} D$.)

\section{A.2.3 Projectors}

Suppose we have a $N^{2} \times N^{2}$-dimensional numerical $\mathrm{R}$-matrix which satisfies a characteristic equation of the form

$$
\prod_{a=1}^{m}\left(\hat{R}-\mu_{a} I\right)=0
$$

where $\left\{\mu_{a} \mid a=1, \ldots, m\right\}$ are the $m$ distinct eigenvalues of $\hat{R}$. We may therefore define $m$ projectors $P_{i}$ as

$$
P_{a}:=\prod_{b \neq a}\left(\frac{\mu_{b} I-\hat{R}}{\mu_{b}-\mu_{a}}\right) .
$$


These projectors satisfy

$$
P_{a} P_{b}=\delta_{a b} P_{a}, \quad \hat{R}^{M}=\sum_{a} \mu_{a}^{M} P_{a} .
$$

Now we turn our attention to the specific case where the R-matrix is that of one of the simple quantum Lie groups discussed above; for $G L_{q}(N)$ and $S L_{q}(N)$, there are two projectors $P_{+}$and $P_{-}$, given by

$$
P_{+}=\frac{q^{-1} I+r^{-1} \hat{R}}{q+q^{-1}}, \quad P_{-}=\frac{q I-r^{-1} \hat{R}}{q+q^{-1}},
$$

corresponding to the symmetrizer and antisymmetrizer respectively.

For $S O_{q}(N)$ and $S P_{q}\left(\frac{1}{2} N\right)$, there are three projectors:

$$
\begin{aligned}
& P_{1}=\frac{\left(q^{-1} I+\hat{R}\right)\left(\epsilon q^{\epsilon-N} I-\hat{R}\right)}{\left(q+q^{-1}\right)\left(\epsilon q^{\epsilon-N}-q\right)}, \quad P_{2}=\frac{(q I-\hat{R})\left(\epsilon q^{\epsilon-N} I-\hat{R}\right)}{\left(q+q^{-1}\right)\left(\epsilon q^{\epsilon-N}+q^{-1}\right)}, \\
& P_{0}=\frac{(q I-\hat{R})\left(q^{-1} I+\hat{R}\right)}{\left(q-\epsilon q^{\epsilon-N}\right)\left(\epsilon q^{\epsilon-N}+q^{-1}\right)} .
\end{aligned}
$$

Remember that there exists a metric for both these cases; it is defined as that matrix $g^{i j}$ which satisfies

$$
\begin{aligned}
& \left(P_{1}\right)^{i j}{ }_{k \ell} g^{k \ell}=\left(P_{2}\right)^{i j}{ }_{k \ell} g^{k \ell}=0 \\
& \left(P_{0}\right)^{i j}{ }_{k \ell} g^{k \ell}=g^{i j}
\end{aligned}
$$

For $S O_{q}(N), P_{1}$ projects out the symmetric traceless subspace, $P_{2}$ the antisymmetric subspace, and $P_{0}$ the trace; for $S P_{q}\left(\frac{1}{2} N\right)$, the metric is now antisymmetric, and $P_{1}$ and $P_{2}$ switch roles. $g^{i j}$ is invertible (with inverse $g_{i j}$ ), and in terms of the unit matrices in the fundamental representation takes the form

$$
g=\sum_{I} q^{-\rho_{I}} E_{I I^{\prime}}
$$

so that $D^{i}{ }_{j}=g^{i k} g_{j k}$. 


\section{Appendix B}

\section{Classical Differential Geometry}

We present here a quick review of the "Cartan calculus" on a classical differentiable manifold; for a more in-depth treatment of the subject, there are several texts which the reader may refer him/herself to $[34,35,36]$.

\section{B.1 The Tangent Space}

Let $M$ be a $C^{\infty} \mathrm{N}$-dimensional real differentiable manifold; the unital associative algebra $\operatorname{Fun}(M)$ is the space of all $C^{\infty}$ functions which map $M$ to $\mathbf{R}$, with addition, multiplication, and unit given by

$$
(f+g)(m)=f(m)+g(m), \quad(f g)(m)=f(m) g(m), \quad 1(m)=1 .
$$

for $m \in M, f, g \in \operatorname{Fun}(M)$.

For each subset $S \subseteq M$ we can define an equivalence relation $\stackrel{S}{\cong}$ on $\operatorname{Fun}(M)$ : two functions $f, g \in \operatorname{Fun}(M)$ satisfy $f \stackrel{S}{\cong} g$ if there exists an open set $U \subseteq M$ containing $S$ such that $\left.f\right|_{U}=\left.g\right|_{U} . f_{S}$, the germ of $f$ on $S$, is defined as the equivalence class of $f$ under $\stackrel{S}{\cong}$, and $\mathcal{F}_{S}$ is the space of all such classes (also a unital associative algebra). We may then introduce $T_{S}(M)$, the tangent space of $M$ on $S$, as the vector space over $\mathbf{R}$ consisting of derivations on $\mathcal{F}_{S}$, i.e. linear maps from $\mathcal{F}_{S}$ into itself such that for $X_{S} \in T_{S}(M)$ and $f_{S}, g_{S} \in \mathcal{F}_{S}$,

$$
X_{S}\left(f_{S} g_{S}\right)=X_{S}\left(f_{S}\right) g_{S}+f_{S} X_{S}\left(g_{S}\right), \quad X_{S}\left(1_{S}\right) \equiv 0
$$


This space is a Lie algebra, with the commutator being defined through

$$
\left[X_{S}, Y_{S}\right]\left(f_{S}\right):=X_{S}\left(Y_{S}\left(f_{S}\right)\right)-Y_{S}\left(X_{S}\left(f_{S}\right)\right) \text {. }
$$

As an example, take $S$ to be the single point $m$, and let $\left\{x^{\mu} \mid \mu=1, \ldots, N\right\}$ be a local coordinate system at $m . T_{m}(M)$ is a vector space over $\mathcal{F}_{m}$ with basis $\left\{\left(\partial_{\mu}\right)_{m}\right\}$, where

$$
\left(\partial_{\mu}\right)_{m}\left(f_{m}\right):=\left(\frac{\partial f}{\partial x^{\mu}}\right)_{m}
$$

Thus, $T_{m}(M)$ consists of differential operators on functions at $m$.

\section{B.2 The Exterior Derivative}

We now assume the existence of a linear map $\epsilon_{S}: \mathcal{F}_{S} \rightarrow \mathbf{R}$ (for instance, $\epsilon_{S}\left(f_{S}\right):=$ $\int_{S} f$ is such a map). The existence of such a map allows the definition of a linear map $\delta_{0}: \mathcal{F}_{S} \rightarrow T_{S}^{*}(M)$ as

$$
\left(\delta_{0}\left(f_{S}\right), X_{S}\right):=\epsilon_{S}\left(X_{S}\left(f_{S}\right)\right)
$$

where $T_{S}^{*}(M)$, the cotangent space on $S$, is the dual of $T_{S}(M)$, and $($,$) is the inner$ product pairing the two spaces. Notice that this inner product is degenerate; if - either $f_{S} \propto 1_{S}$ or $\epsilon_{S}\left(X_{S}\left(f_{S}\right)\right)$ vanishes, then the inner product above will be zero as well. However, if we define $\mathcal{F}_{S}^{1}:=\operatorname{ker} \epsilon_{S}$ and $\mathcal{F}_{S}^{2}:=\left\{f_{S} \in \mathcal{F}_{S}^{1} \mid X_{S}\left(f_{S}\right) \in \mathcal{F}_{S}^{1} \forall X_{S} \in\right.$ $\left.T_{S}(M)\right\}$, then (B.5) will vanish for $f_{S} \in \mathcal{F}_{S}^{1} / \mathcal{F}_{S}^{2}$ iff either $f_{S} \equiv 0$ or $X_{S} \equiv 0$. Thus, when $($,$) is restricted to \delta_{0}\left(\mathcal{F}_{S}^{1} / \mathcal{F}_{S}^{2}\right) \otimes T_{S}(M) \rightarrow \mathbb{R}$, the inner product is nondegenerate, and $\delta_{0}: \mathcal{F}_{S}^{1} / \mathcal{F}_{S}^{2} \rightarrow T_{S}^{*}(M)$ is a bijective map.

We define the $\mathcal{F}_{S}$-bimodule $\Gamma_{S}$ to be the space spanned by elements of the form $f_{S} \delta_{0}\left(g_{S}\right)$ and $\delta_{0}\left(f_{S}\right) g_{S}$ with $f_{S}, g_{S} \in \mathcal{F}_{S}$, where

$$
\left(f_{S} \delta_{0}\left(g_{S}\right), X_{S}\right):=\epsilon_{S}\left(f_{S} X_{S}\left(g_{S}\right)\right), \quad\left(\delta_{0}\left(f_{S}\right) g_{S}, X_{S}\right):=\epsilon_{S}\left(X_{S}\left(f_{S}\right) g_{S}\right)
$$

These lead to the following important result:

$$
\begin{aligned}
\left(\delta_{0}\left(f_{S} g_{S}\right), X_{S}\right) & =\epsilon_{S}\left(X_{S}\left(f_{S} g_{S}\right)\right) \\
& =\epsilon_{S}\left(X_{S}\left(f_{S}\right) g_{S}+f_{S} X_{S}\left(g_{S}\right)\right) \\
& =\left(\delta_{0}\left(f_{S}\right) g_{S}+f_{S} \delta_{0}\left(g_{S}\right), X_{S}\right)
\end{aligned}
$$


so $\delta_{0}$ satisfies the familiar Leibniz rule on functions. Note that this implies that $\delta_{0}\left(f_{S}\right) g_{S} \equiv \delta_{0}\left(f_{S} g_{S}\right)-f_{S} \delta_{0}\left(g_{S}\right)$, so even though $\Gamma_{S}$ was defined as a bimodule, it may be thought of as $\mathcal{F}_{S} \delta_{0}\left(\mathcal{F}_{S}^{1} / \mathcal{F}_{S}^{2}\right)$.

We can define the space of $p$-forms $\Lambda_{S}^{p}$ to be the span over $\mathbf{R}$ of elements of the form $f_{S}^{(0)} \delta_{0}\left(f_{S}^{(1)}\right) \wedge \ldots \wedge \delta_{0}\left(f_{S}^{(\bar{p})}\right)$, where $f_{S}^{(0)} \in \mathcal{F}_{S}, f_{S}^{(k)} \in \mathcal{F}_{S}^{1} / \mathcal{F}_{S}^{2}, k=1, \ldots, p$ (so $\Lambda_{S}^{0} \equiv \mathcal{F}_{S}$ and $\left.\Lambda_{S}^{1} \equiv \Gamma_{S}\right)$ and the wedge product $\wedge$ is defined such that $\delta_{0}\left(f_{S}\right) \wedge$ $\delta_{0}\left(g_{S}\right) \in\left(T_{S}^{*}(M)\right)^{\otimes 2}$ satisfies

$$
\left(\delta_{0}\left(f_{S}\right) \wedge \delta_{0}\left(g_{S}\right), X_{S} \otimes Y_{S}\right)=\left(\delta_{0}\left(f_{S}\right), X_{S}\right)\left(\delta_{0}\left(g_{S}\right), Y_{S}\right)-\left(X_{S} \leftrightarrow Y_{S}\right),
$$

and analogously for forms of higher degree (i.e. total antisymmetrization with respect to the vector fields). $\delta_{p}: \Lambda_{S}^{p} \rightarrow \Lambda_{S}^{p+1}$ is then defined as the linear map

$$
\delta_{p}\left(f_{S}^{(0)} \delta_{0}\left(f_{S}^{(1)}\right) \wedge \ldots \wedge \delta_{0}\left(f_{S}^{(p)}\right)\right):=\delta_{0}\left(f_{S}^{(0)}\right) \wedge \delta_{0}\left(f_{S}^{(1)}\right) \wedge \ldots \wedge \delta_{0}\left(f_{S}^{(p)}\right)
$$

so $\delta_{p}$ satisfies $\delta_{p+1} \delta_{p} \equiv 0$. (From this point onward, we will suppress the wedge product, with its presence being assumed whenever we multiply any $p$-forms together.)

We can now define the exterior algebra on $S$ to be the direct sum of each of the spaces of $p$-forms, i.e. $\Lambda_{S}(M):=\bigoplus_{p} \wedge_{S}^{p}$. The multiplication between elements of this space is just the wedge product, so the product of a $p$-form $\phi_{S}$ and a $q$-form $\psi_{S}$ is a $(p+q)$-form. We may extend $\delta_{0}$ to a linear map $\delta$ on all of $\Lambda_{S}(M)$ by defining

$$
\begin{aligned}
\delta\left(1_{S}\right) & =0 \\
\delta^{2}\left(\phi_{S}\right) & =0 \\
\delta\left(\phi_{S} \psi_{S}\right) & =\delta\left(\phi_{S}\right) \psi_{S}+(-1)^{p} \phi_{S} \delta\left(\psi_{S}\right)
\end{aligned}
$$

where $\phi_{S}$ is a $p$-form. This map is called the exterior derivative on $\Lambda_{S}(M)$, and is a derivation of degree +1 (a map of degree $d$ maps $\bigwedge_{S}^{p}$ into $\bigwedge_{S}^{p+d}$ ).

\section{B.3 The Inner Derivation}

We now introduce another linear map from $\Lambda_{S}(M)$ into itself, the inner derivation $i$, which in effect "undoes" the exterior derivative, in the sense that it maps $p$-forms to $(p-1)$-forms. It takes as an argument a vector field $X_{S} \in T_{S}(M)$, and is first defined on 0 - and 1 -forms:

$$
i_{X_{S}}\left(f_{S}\right):=0 \quad i_{X_{S}}\left(f_{S} \delta_{0}\left(g_{S}\right)\right):=f_{S} X_{S}\left(g_{S}\right)
$$


Its action on a $p$-form $\phi_{S}$ for $p>1$ is given via

$$
\begin{gathered}
\left(i_{X_{S}}\left(\phi_{S}\right), Y_{S}^{(1)} \otimes \ldots \otimes Y_{S}^{(p-1)}\right)= \\
\left(\phi_{S}, X_{S} \otimes Y_{S}^{(1)} \otimes \ldots \otimes Y_{S}^{(p-1)}\right)-\left(\phi_{S}, Y_{S}^{(1)} \otimes X_{S} \otimes \ldots \otimes Y_{S}^{(p-1)}\right)+\ldots \\
\quad+(-1)^{p-1}\left(\phi_{S}, Y_{S}^{(1)} \otimes \ldots \otimes Y_{S}^{(p-1)} \otimes X_{S}\right)
\end{gathered}
$$

so, for example,

$$
i_{X_{S}}\left(f_{S}^{(0)} \delta_{0}\left(f_{S}^{(1)}\right) \delta_{0}\left(f_{S}^{(2)}\right)\right)=f_{S}^{(0)} X_{S}\left(f_{S}^{(1)}\right) \delta_{0}\left(f_{S}^{(2)}\right)-f_{S}^{(0)} \delta_{0}\left(f_{S}^{(1)}\right) X_{S}\left(f_{S}^{(2)}\right)
$$

From the above, it is easily seen that the inner derivation is a derivation of degree -1 .

\section{B.4 The Lie Derivative and the Graded Deriva- tion Algebra}

It is now straightforward to define the Lie Derivative $\mathcal{L}$ as a linear map from the exterior algebra into itself which takes $p$-forms to $p$-forms. It is defined on a $p$-form $\phi_{S}$ by

$$
\mathcal{L}_{X_{S}}\left(\phi_{S}\right):=\delta\left(i_{X_{S}}\left(\phi_{S}\right)\right)+i_{X_{S}}\left(\delta\left(\phi_{S}\right)\right)
$$

and is therefore a derivation of degree 0 . From this definition, it immediately follows that $\mathcal{L}_{X_{S}}$ acts as $X_{S}$ on 0 -forms, and on 1 -forms as

$$
\mathcal{L}_{X_{S}}\left(f_{S} \delta_{0}\left(g_{S}\right)\right)=X_{S}\left(f_{S}\right) \delta_{0}\left(g_{S}\right)+f_{S} \delta_{0}\left(X_{S}\left(g_{S}\right)\right) .
$$

The utility of introducing the Lie derivative via the definition (B.14) lies in the fact that with its inclusion, the three derivations generate a graded algebra, the Cartan calculus, whose (anti)commutation relations are

$$
\begin{array}{cll}
{\left[\delta, \mathcal{L}_{X_{S}}\right]=0,} & \{\delta, \delta\}=0, & \left\{\delta, i_{X_{S}}\right\}=\mathcal{L}_{X_{S}}, \\
{\left[\mathcal{L}_{X_{S}}, i_{Y_{S}}\right]=i_{\left[X_{S}, Y_{S}\right]},} & \left\{i_{X_{S}}, i_{Y_{S}}\right\}=0, & {\left[\mathcal{L}_{X_{S}}, \mathcal{L}_{Y_{S}}\right]=\mathcal{L}_{\left[X_{S}, Y_{S}\right]} .}
\end{array}
$$

These relations, plus the actions of each of the derivations on 0 - and 1-forms, completely specify the differential geometry of the manifold $M$. 


\section{Appendix C}

\section{Differential Calculus on Hopf Algebras}

We present here a review of the standard way to introduce a differential calculus on an arbitrary Hopf algebra. The concepts of noncommutative geometry underlying this treatment were first examined by Connes [2]; a good treatment of these ideas for physicists may be found in [37]. The extension of this general structure to the case of a bicovariant Hopf algebra was dealt with in great detail by Woronowicz [3]. (Much of the material herein will of course be very reminiscent of the classical treatment given in Appendix B.)

\section{C.1 The Universal Differential Calculus}

Let $\mathcal{A}$ be a unital associative algebra over a field $k$, and $\Gamma(\mathcal{A})$ an $\mathcal{A}$-bimodule such that there exists a linear map $\delta: \mathcal{A} \rightarrow \Gamma(\mathcal{A})$ which satisfies the following:

$$
\begin{aligned}
\delta\left(1_{\mathcal{A}}\right) & =0 \\
\delta(a b) & =\delta(a) b+a \delta(b),
\end{aligned}
$$

where $1_{\mathcal{A}}$ is the unit in $\mathcal{A}$, and $a, b \in \mathcal{A}$. Note that the latter of these conditions implies that $\Gamma(\mathcal{A})$ is the span of elements of the form $a \delta(b)$.

As an example of this, take $\Gamma(\mathcal{A}) \subset \mathcal{A} \otimes \mathcal{A}$ as the kernel of the multiplication on $\mathcal{A}$, i.e. the span of elements of the form $\sum_{i} a_{i} \otimes b_{i}$ where $\sum_{i} a_{i} b_{i}=0 . \quad \Gamma(\mathcal{A})$ is made into an $\mathcal{A}$-bimodule by defining left and right multiplication by $\mathcal{A}$ to be 
$c\left(\sum_{i} a_{i} \otimes b_{i}\right)=\sum_{i}\left(c a_{i}\right) \otimes b_{i}$ and $\left(\sum_{i} a_{i} \otimes b_{i}\right) c=\sum_{i} a_{i} \otimes\left(b_{i} c\right), c \in \mathcal{A}$. The map $\delta$ which satisfies all the needed conditions is given by $\delta(a):=1_{\mathcal{A}} \otimes a-a \otimes 1_{\mathcal{A}}$.

We now introduce the differential envelope associated with $\mathcal{A}$, denoted by $\Omega(\mathcal{A})$; it is the algebra which is spanned by elements of $\mathcal{A}$, together with formal products of elements of $\Gamma(\mathcal{A})$ modulo the relations (C.1), namely, elements of the form $a_{0} \delta\left(a_{1}\right) \delta\left(a_{2}\right) \ldots \delta\left(a_{p}\right)$. Such elements are called $p$-forms $(e . g$. 0 -forms are elements of $\mathcal{A}, 1$-forms elements of $\Gamma(\mathcal{A})$, etc. $\Omega(\mathcal{A})$ is easily seen to be associative and unital (with unit $1=1_{\mathcal{A}}$ ); furthermore, $\delta$ can be extended to a linear map $\delta: \Omega(\mathcal{A}) \rightarrow \Omega(\mathcal{A})$ by requiring

$$
\begin{aligned}
\delta(1) & =0 \\
\delta^{2}(\phi) & =0 \\
\delta(\phi \psi) & =\delta(\phi) \psi+(-1)^{p} \phi \delta(\psi)
\end{aligned}
$$

where $\phi, \psi \in \Omega(\mathcal{A}), \phi$ a $p$-form. Thus, $\delta$ maps $p$-forms to $(p+1)$-forms. $\delta$ is the exterior derivative on $\Omega(\mathcal{A})$, and we call $(\Omega(\mathcal{A}), \delta)$ the universal differential calculus (UDC) associated with $\mathcal{A}$.

If $\mathcal{C}$ is a Hopf algebra which coacts on $\mathcal{A}$ as explained in Chapter 3.3, we may extend the right coaction to $\Omega(\mathcal{A})$ by requiring that $\Delta_{\mathcal{C}}: \Omega(\mathcal{A}) \rightarrow \Omega(\mathcal{A}) \otimes \mathcal{C}$ satisfies

$$
\begin{aligned}
\Delta_{\mathcal{C}}(\delta(a)) & =(\delta \otimes \mathrm{id}) \Delta_{\mathcal{C}}(a) \\
\Delta_{\mathcal{C}}(\phi \psi) & =\Delta_{\mathcal{C}}(\phi) \Delta_{\mathcal{C}}(\psi),
\end{aligned}
$$

for $a \in \mathcal{A}, \phi, \psi \in \Omega(\mathcal{A})$. The left coaction may be extended analogously.

\section{C.1.1 The Universal Differential Calculus of a Hopf Alge- bra}

Up to this point, we have said nothing about $\mathcal{A}$ being anything more than a unital associative algebra. However, if we now allow $\mathcal{A}$ to be a Hopf algebra, the UDC acquires more structure [3]. For instance, we may extend the natural coactions $\Delta_{\mathcal{A}}={ }_{\mathcal{A}} \Delta=\Delta$ of $\mathcal{A}$ on itself as described above. Thus, for each $a \in \mathcal{A}$, the CartanMaurer form $\omega_{a}:=S\left(a_{(1)}\right) \delta\left(a_{(2)}\right)$ is both left-invariant and right-covariant under these coactions, i.e.

$$
{ }_{\mathcal{A}} \Delta\left(\omega_{a}\right)=1_{\mathcal{A}} \otimes \omega_{a}, \quad \Delta_{\mathcal{A}}\left(\omega_{a}\right)=\omega_{a_{(2)}} \otimes S\left(a_{(1)}\right) a_{(3)} .
$$


(The latter of these shows explicitly the appearance of the adjoint coaction (3.23).) Furthermore, its differential has the particularly nice form

$$
\delta\left(\omega_{a}\right)=-\omega_{a_{(1)}} \omega_{a_{(2)}} .
$$

A further consequence of $\mathcal{A}$ being a Hopf algebra is that $\mathcal{A} \equiv k 1_{\mathcal{A}} \oplus \mathcal{K}$, where $k 1_{\mathcal{A}}$ is shorthand for the subspace of all elements proportional to the unit, and $\mathcal{K}:=\operatorname{ker} \epsilon$. $\delta$ and $\omega$ thus vanish on $k 1_{\mathcal{A}}$, so both may be restricted to acting on $\mathcal{K}$ only. Since $a \delta(b)=a b_{(1)} \omega_{b_{(2)}}$, any element of $\Gamma(\mathcal{A})$ may be written in terms of Cartan-Maurer forms with coefficients in $\mathcal{A}$. Therefore, we take $\Omega(\mathcal{A})$ to be the span of $p$-forms $a_{0} \omega_{a_{1}} \ldots \omega_{a_{p}}$, with $a_{0} \in \mathcal{A}$ and $a_{i} \in \mathcal{K}, i=1, \ldots, p$.

It is possible to impose a ${ }^{*}$-Hopf algebra structure on the UDC when $\mathcal{A}$ itself is a *-Hopf algebra [38]. This is accomplished by giving the following compatibility conditions: if $\phi \in \Omega(\mathcal{A})$ is a $p$-form, then

$$
\begin{aligned}
\Delta(\delta \phi) & =\left(\delta \otimes \mathrm{id}+(-1)^{p} \mathrm{id} \otimes \delta\right) \Delta(\phi) \\
\epsilon(\delta \phi) & =0 \\
S(\delta \phi) & =\delta(S(\phi)), \\
\theta(\delta \phi) & =\delta(\theta(\phi)),
\end{aligned}
$$

provided that the multiplication on the tensor product $\Omega(\mathcal{A}) \otimes \Omega(\mathcal{A})$ is $\mathbf{Z}_{2}$-graded:

$$
\left(\phi_{1} \otimes \phi_{2}\right)\left(\psi_{1} \otimes \psi_{2}\right)=(-1)^{p q}\left(\phi_{1} \psi_{1} \otimes \phi_{2} \psi_{2}\right),
$$

where $\phi_{2}$ and $\psi_{1}$ are $p$-and $q$-forms, respectively. It is easily shown that these conditions are consistent with both the defining relations of a *-Hopf algebra and those of the UDC. An interesting result of these relations is that the coproduct of a Cartan-Maurer form is related to the right and left coactions by

$$
\Delta\left(\omega_{a}\right)=\left({ }_{\mathcal{A}} \Delta+\Delta_{\mathcal{A}}\right)\left(\omega_{a}\right)
$$

(where the elements of $\mathcal{A}$ appearing in the right-hand side of this relation are to be taken as 0 -forms in $\Omega(\mathcal{A})$; of course).

\section{C.2 General Differential Calculus}

So far, the only commutation relations we have in $\Omega(\mathcal{A})$ are those which follow from (C.2); we assume nothing else. Here we review the standard method of introducing 
nontrivial commutation relations into the differential envelope which maintains the covariance properties we have chosen (e.g. left-invariance of $\omega_{a}$ ).

Suppose that $\mathcal{A}$ is a Hopf algebra such that there exists a subalgebra $\mathcal{M} \subset \mathcal{A}$ satisfying

1. $\mathcal{M} \subseteq \mathcal{K}$,

2. $\mathcal{M A} \subseteq \mathcal{M}$

3. $\Delta^{\mathrm{Ad}}(\mathcal{M}) \subseteq \mathcal{M} \otimes \mathcal{A}$.

We define the submodule $\mathcal{N}_{\mathcal{M}} \subseteq \Gamma(\mathcal{A})$ as the space spanned by 1 -forms of the form $a \omega_{m}$, where $a \in \mathcal{A}$ and $m \in \mathcal{M}$. The above properties of $\mathcal{M}$ imply properties of $\mathcal{N}_{\mathcal{M}}$ : (1) and (2) give $\mathcal{N}_{\mathcal{M}} \mathcal{A} \subseteq \mathcal{N}_{\mathcal{M}}$, and (3) gives $\Delta_{\mathcal{A}}\left(\mathcal{N}_{\mathcal{M}}\right) \subseteq \mathcal{N}_{\mathcal{M}} \otimes \mathcal{A}$. Such an $\mathcal{M}$ always exists; $\{0\}$ and $\mathcal{K}$ both satisfy all three conditions.

With $\mathcal{M}$ as above, we can construct the $\mathcal{A}$-module $\Gamma_{\mathcal{M}}:=\Gamma(\mathcal{A}) / \mathcal{N}_{\mathcal{M}}$. When $\mathcal{M}=\{0\}$, and therefore $\mathcal{N}_{\mathcal{M}}=\{0\}$, the only commutation relations between elements of $\mathcal{A}$ and $\Gamma_{\mathcal{M}}$ are those allowed by the Leibniz rule, and we recover the UDC; when $\mathcal{M}=\mathcal{K}, \mathcal{N}_{\mathcal{M}}=\Gamma(\mathcal{A})$, so $\Gamma_{\mathcal{M}}=\{0\}$, and we end up with a trivial differential calculus. However, if there exists an $\mathcal{M}$ in between these two extreme cases, then there exist additional commutation relations between elements of $\Gamma_{\mathcal{M}}$, namely those given by $\omega_{m} \simeq 0$ for $m \in \mathcal{M}(\simeq$ being the equivalence relation in $\left.\Gamma_{\mathcal{M}}\right)$. Furthermore, we find explicit commutation relations between elements of $\Gamma_{\mathcal{M}}$ by using (C.5) and the properties of $\mathcal{M}$, i.e. $\omega_{m_{(1)}} \omega_{m_{(2)}} \simeq 0$. Therefore, we no longer have a UDC, but rather a differential envelope with nontrivial commutation relations which is constructed using $\mathcal{A}$ and $\Gamma_{\mathcal{M}}$; we refer to this envelope as $\Omega_{\mathcal{M}}$, and the pair $\left(\Omega_{\mathcal{M}}, \delta\right)$ is referred to as the general differential calculus (GDC) associated with $\mathcal{A}$ and $\mathcal{M}$. 